Programs for Simplifying the Analysis of Geographic Information in U.S. Geological Survey Ground-Water Models

By Richard B. Winston

U.S. GEOLOGICAL SURVEY

Open-File Report 01-392 
U.S. DEPARTMENT OF THE INTERIOR

GALE A. NORTON, Secretary

U.S. GEOLOGICAL SURVEY

Charles G. Groat, Director

Although the computer program described in this report has been tested and used by the U.S. Geological Survey (USGS), no warranty, expressed or implied, is made by the USGS as to the accuracy of the functioning of the program and related material. The code may be updated and revised periodically.

Any use of trade, product, or firm names in this publication is for descriptive purposes only and does not imply endorsement by the U.S. Government.

For additional information write to:

Office of Ground Water U.S. Geological Survey

411 National Center

Reston, VA 20192
Copies of this report can be purchased from:

U.S. Geological Survey

Branch of Information Services

Box 25286

Denver, Colorado 80225-0286 


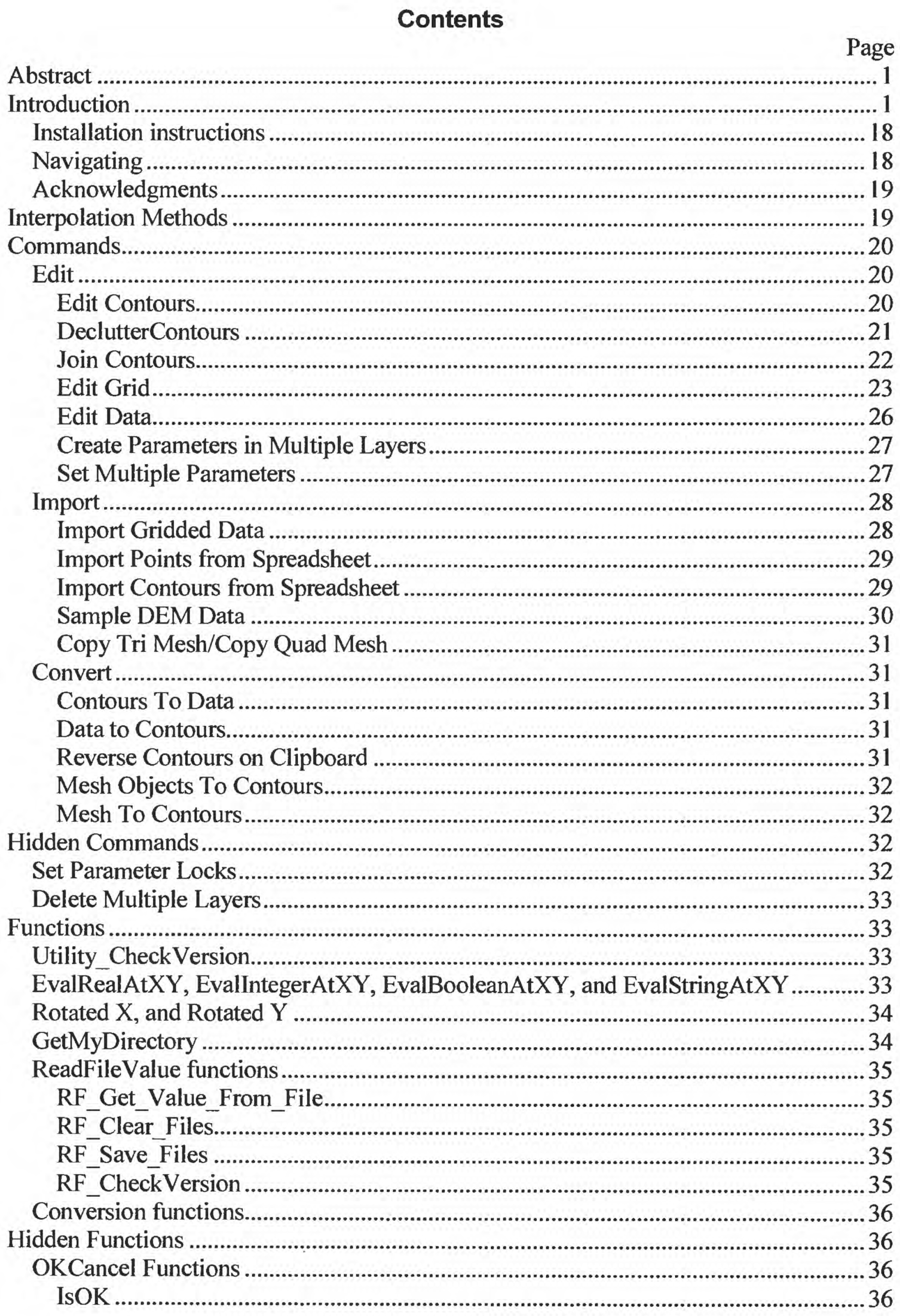




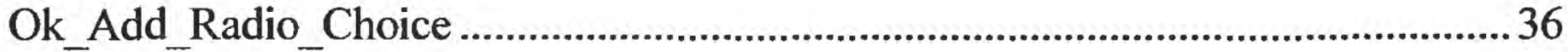

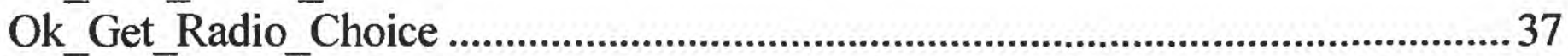

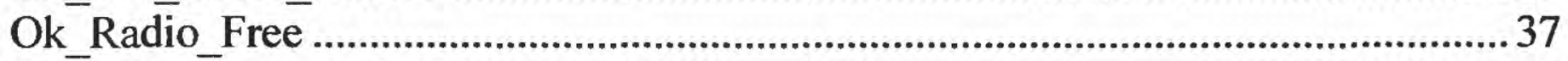

Ok_UserFloat and Ok_UserInteger....................................................................... 37

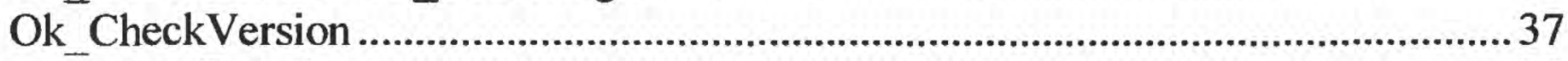

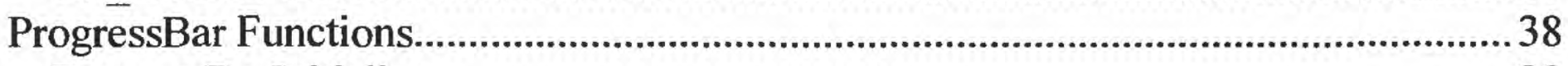

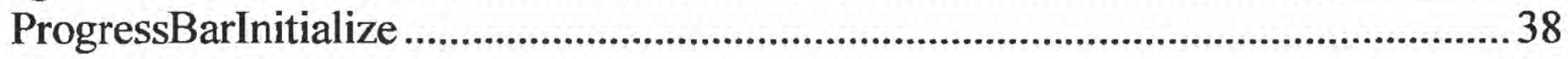

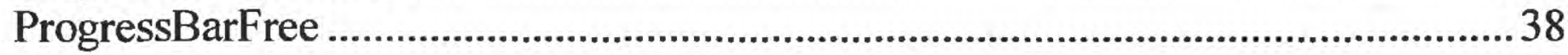

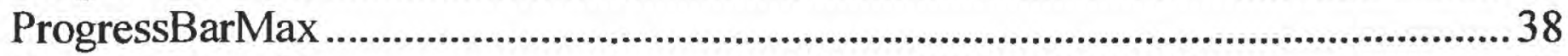

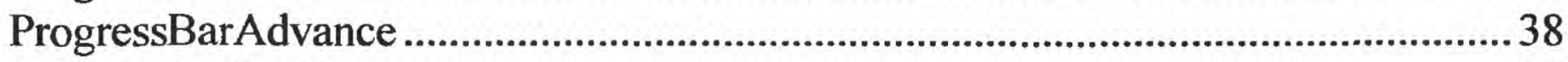

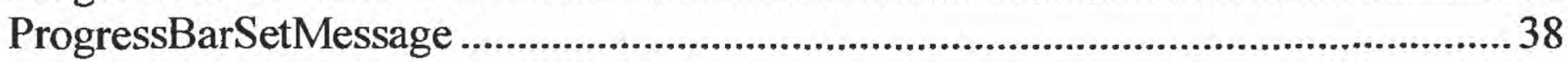

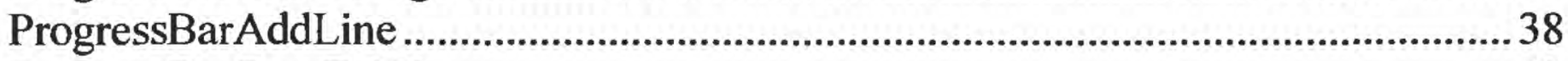

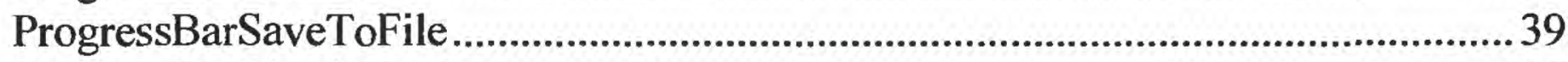

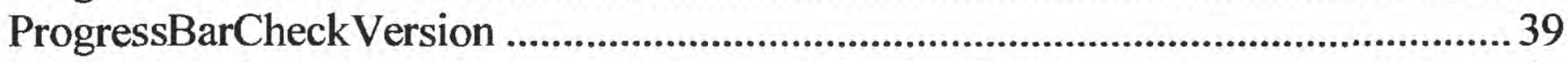

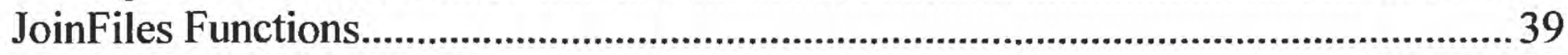

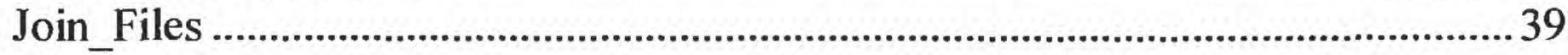

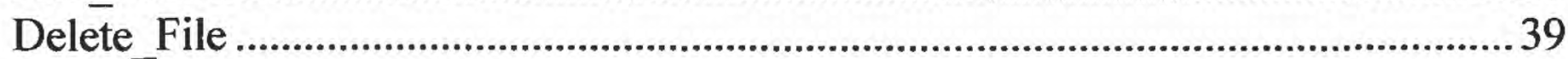

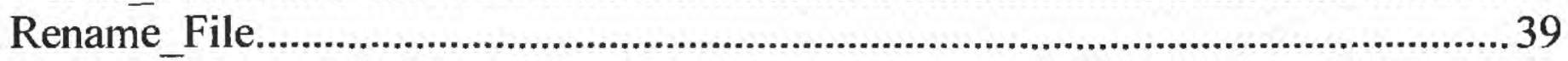

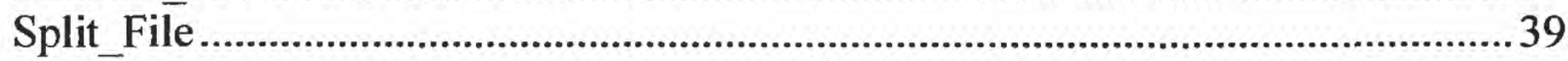

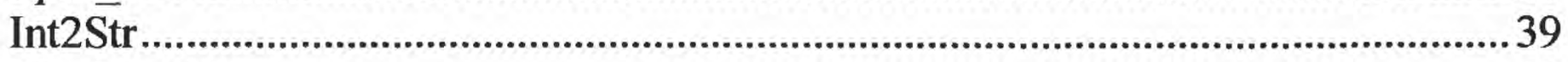

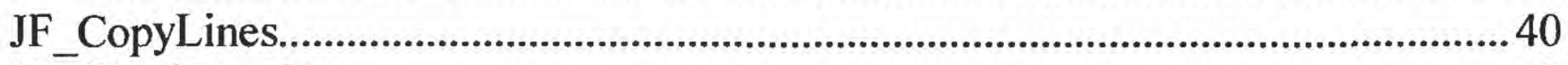

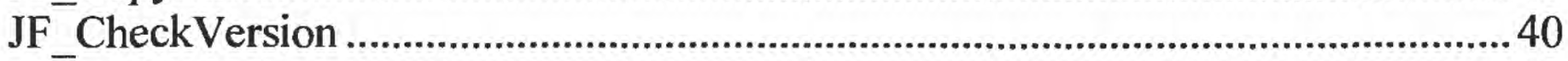

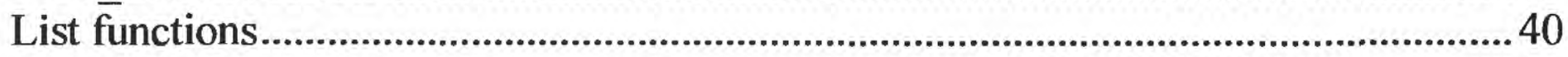

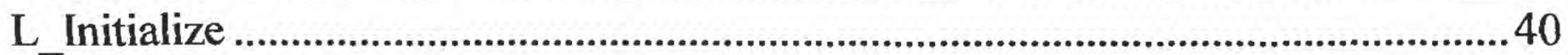

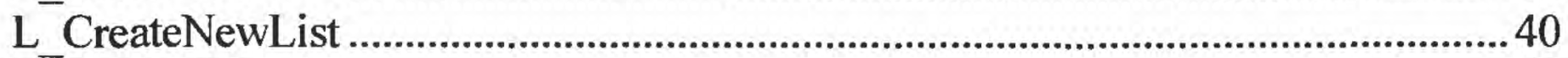

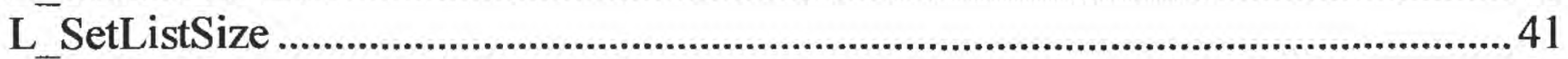

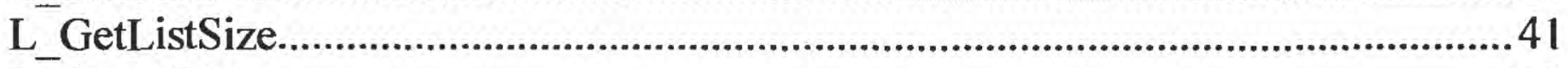

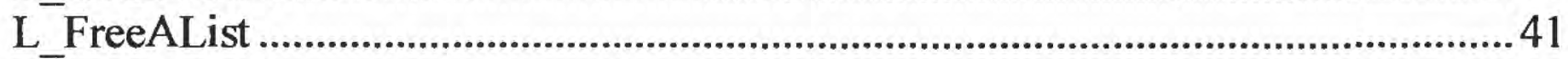

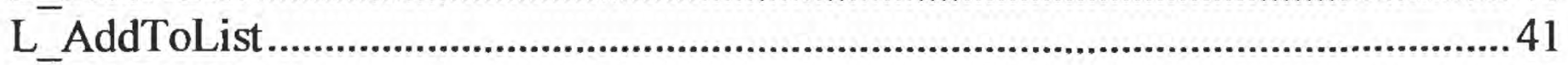

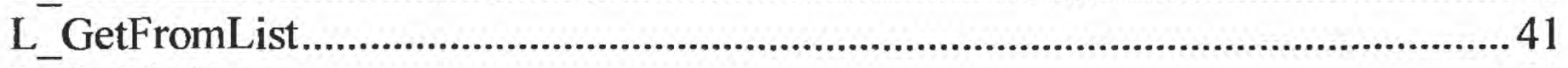

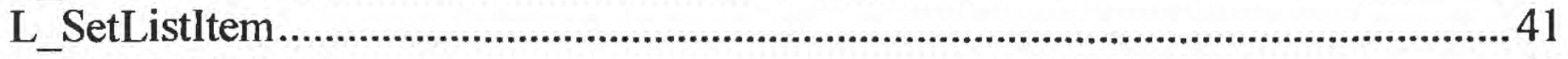

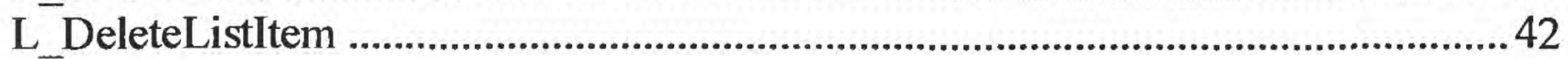

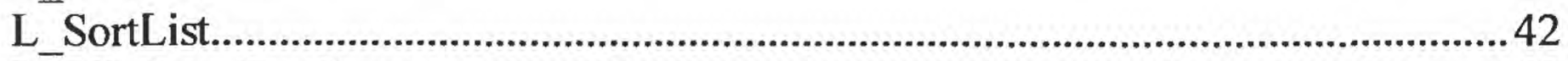

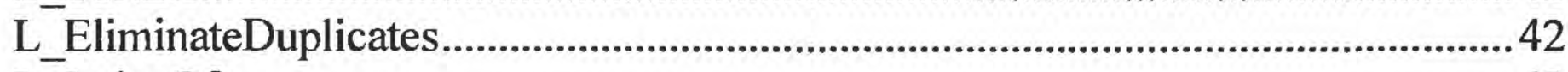

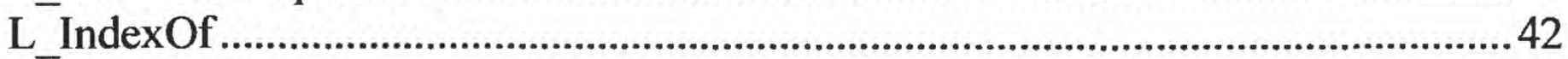

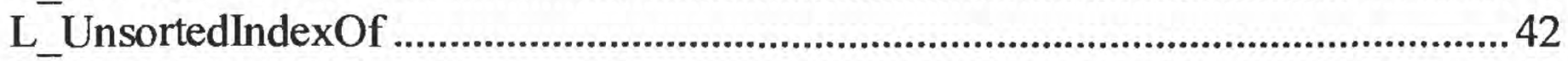

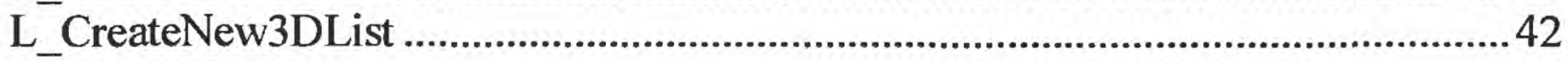

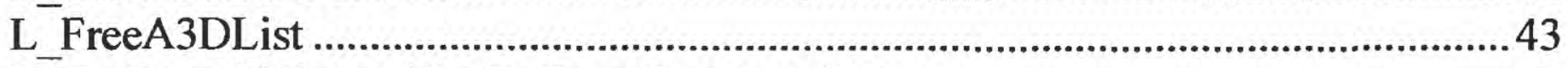

L_GetFrom3Dlist and L_GetFromOneBased3DList ..........................................43

L_Set3DlistItem and L_SetOneBased3DListItem ...............................................43

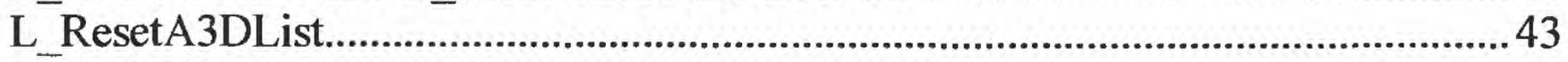

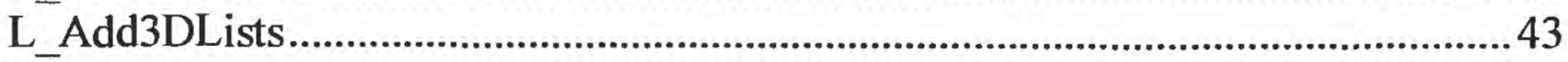

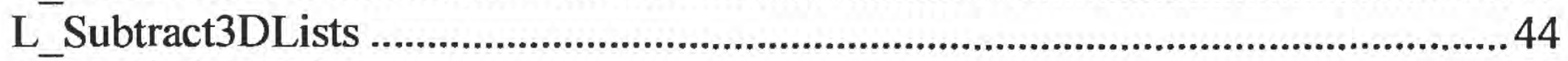

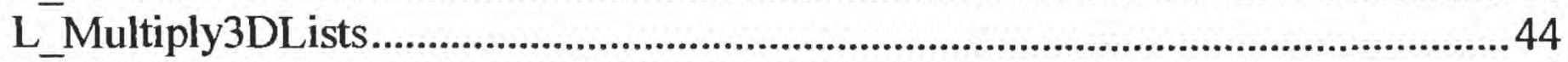

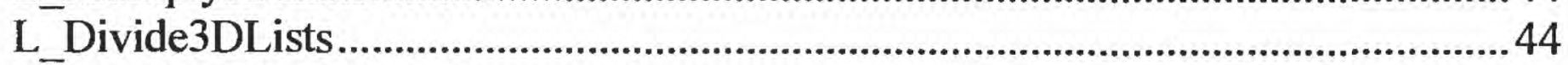

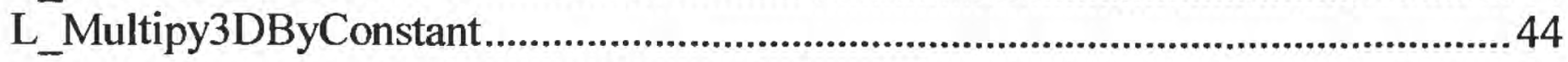




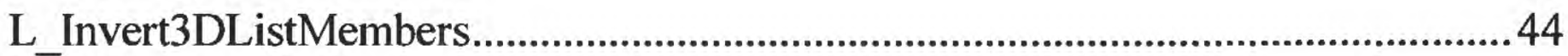

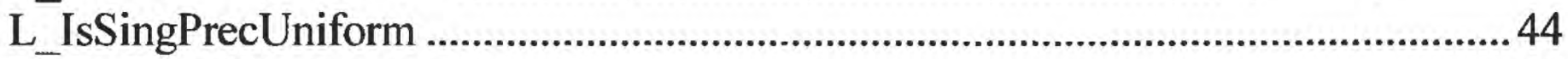

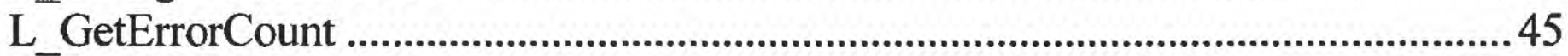

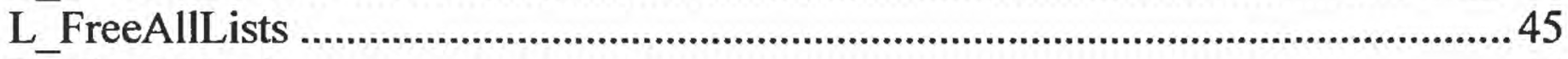

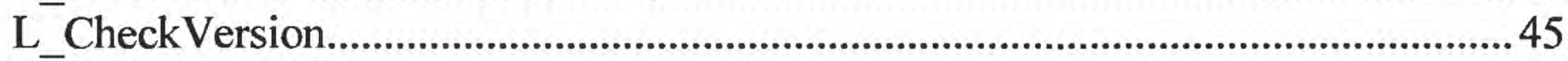

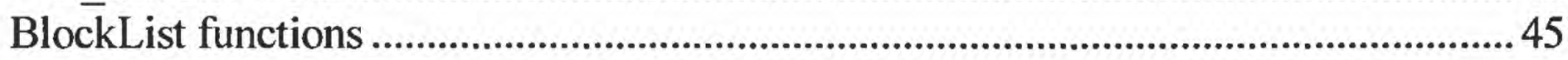

BL_InitializeGridInformation .............................................................................. 46

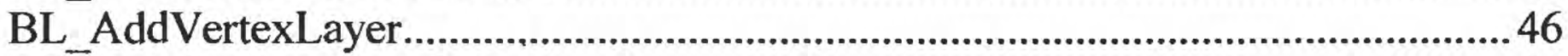

BL_ReInitializeVertexList ........................................................................... 46

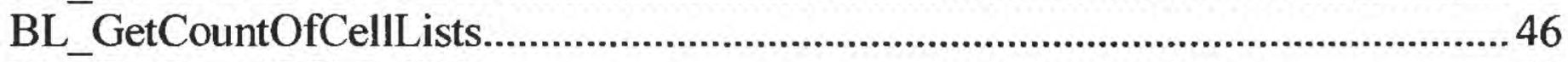

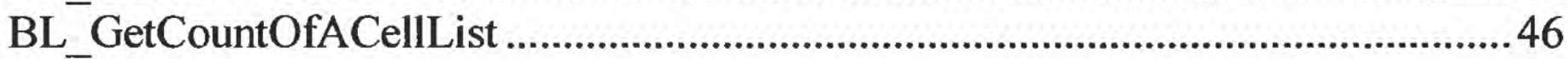

BL_GetCellRow and BL_GetCellColumn.................................................................4

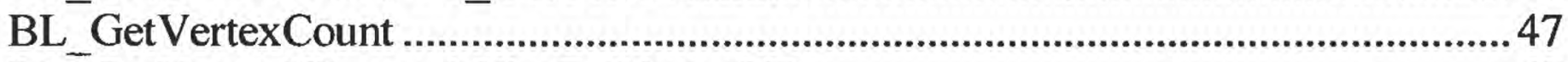

BL_GetVertexXPos and BL_GetVertexYPos .............................................................4

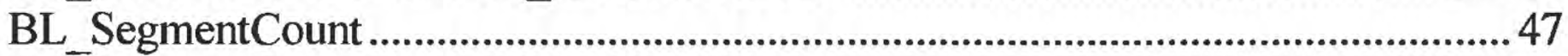

BL_SegmentFirstX and BL_SegmentFirstY ………...................................... 47

BL_SegmentSecondX and BL_SegmentSecondY...................................................4 47

BL_SegmentLengthX and BL_SegmentLength $Y$.....................................................4 4

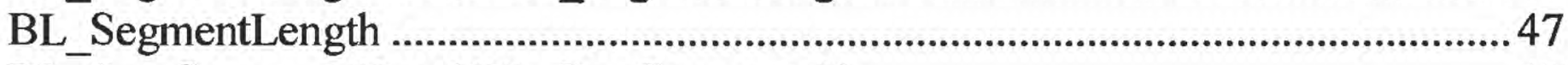

BL_SumSegmentsX and BL_SumSegmentsY …………………………………....47

BL_SumSegmentLengths..................................................................................4

BL_GetCountOfCombinedCellList .................................................................. 48

BL_GetCellRowFromCombinedList and BL_GetCellColumnFromCombinedList 48

BL_GetCountOfCrossRowLists and BL_GetCountOfCrossColumnLists..................48

BL_GetCountOfACrossRowList and BL_GetCountOfACrossColumnList .............48

BL_GetCrossRowRow and BL_GetCrossColumnRow..............................................48

BL_GetCrossRowColumn and BL_GetCrossColumnColumn...................................48

BL_GetCrossRowNeighborColumn and BL_GetCrossColumnNeighborRow ......... 48

BL_GetCrossRowCompositeY and BL_GetCrossColumnCompositeX .....................49

BL_GetSumCrossRowCompositeY and BL_GetSumCrossColumnCompositeX....50

BL_GetCrossRowCompositeLength and BL_GetCrossColumnCompositeLength .50

BL_GetRowBoundary and BL_GetColumnBoundary .............................................50

BL_PointInsideContour ...........................................................................................50

BL_GetRowNodePosition and BL_GetColumnNodePosition .................................50

BL_GetRowBoundaryCount and BL_GetColumnBoundaryCount..............................51

BL_GetRowNodeCount and BL_GetColumnNodeCount .........................................51

BL_GetCellArea........................................................................................

BL_FractionOfLine .....................................................................................

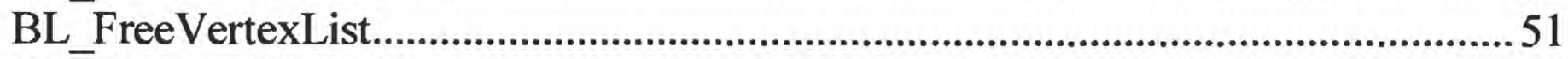

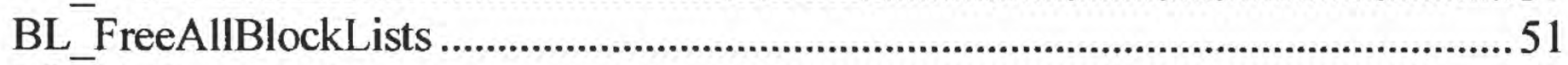

BL_GetErrorCount........................................................................................ 51

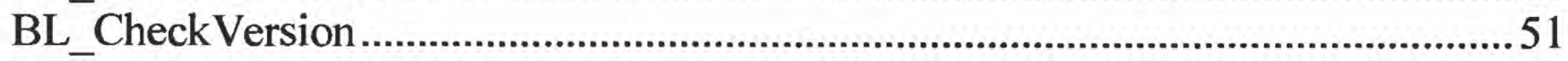

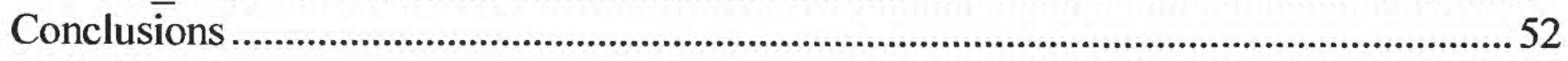

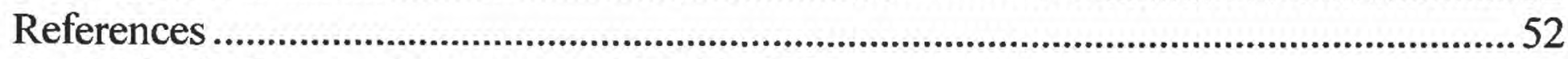

Appendix 1: Custom Components Used in PIEs ..............................................................54

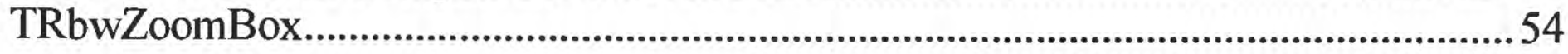




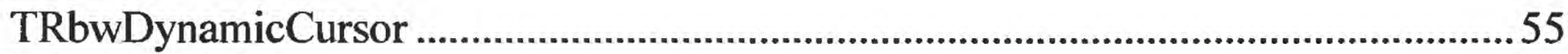

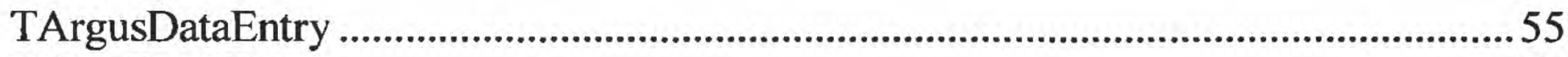

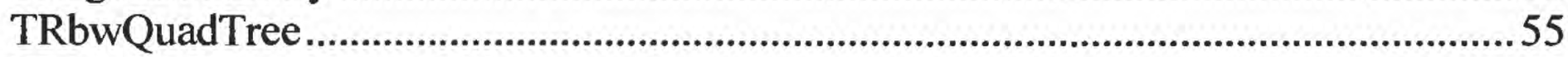

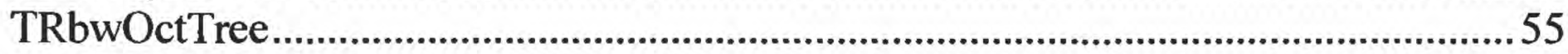

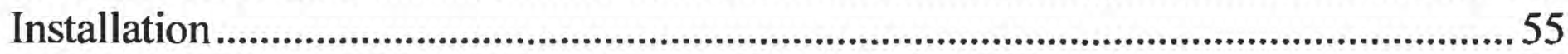

Appendix 2: Example Export Template for ProgressBar PIE.......................................57

Appendix 3: Example Export Template for List PIE ......................................................... 58

Appendix 4: Example Export Template for BlockList PIE ............................................61

Appendix 5: Export Templates Using Rotated X and Rotated Y ....................................64 64

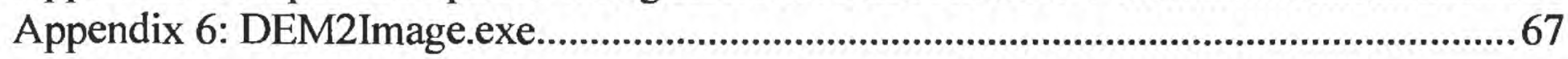

\section{Tables}

Table 1. Commands added to Argus ONE in Utility.dll ................................................ 3

Table 2. Hidden commands added to Argus ONE in Utility.dll ........................................ 3

Table 3. Functions added to Argus ONE ....................................................................... 4

Table 4. Hidden functions added to Argus ONE ........................................................... 10

Figures

Figure 1. Effect of using the Declutter Contours command .......................................... 21

Figure 2. If used improperly, the Declutter Contours command can result in contours that overlap or that no longer show correct geometric relationships............22

Figure 3. Effect of the Join Contours command ............................................................. 23

Figure 4. The cells intercepted by the contour (heavy black line) are shown in dark gray. Those cells which are their neighbors as determined by BL_GetCrossRowNeighborColumn and BL_GetCrossColumnNeighborRow are shown in light gray except for those which are also intercepted by the contour. The heavy dashed line separates each cell intercepted by the contour from its neighbor or neighbors

Figure 5. For the cell indicated by the dark gray square, the length returned by BL_GetCrossRowCompositeY is indicated by the double-headed arrow. To determine this, the function retrieved data not only from the dark gray cell but also from the light gray cells

Figure 6. The result of BL_FractionOfLine for the cell indicated by the dark square would be 0.10 because 10 percent of the contour lies within the cell 


\title{
Programs for Simplifying the Analysis of Geographic Information in U.S. Geological Survey Ground-Water Models
}

\author{
By Richard B. Winston
}

\begin{abstract}
This report describes a number of programs developed at the U.S. Geological Survey (USGS) to enhance existing USGS graphical user-interfaces for ground-water modeling programs. The programs are in the form of dynamic-link libraries that add interpolation methods, commands, and functions to Argus ONE ${ }^{\mathrm{TM}}$ through the use of Plug-In Extensions (PIEs). The interpolation methods, commands, and functions are not specific to any particular type of model but instead may find application under a variety of circumstances. The new interpolation methods provide results much more quickly than the existing interpolation methods in Argus ONE while still giving reasonable results. Some of them also incorporate anisotropy. This may be particularly valuable for cross sectional models. The commands are useful for copying, editing, converting, or importing information. They include commands for creating or modifying grids, editing the precise locations of nodes along contours, importing data as either contours or data points, setting the expressions for multiple parameters, and importing Digital Elevation Models (DEMs). The functions provide convenient methods for evaluating, converting, or storing information. Many of the functions are hidden because they could cause memory leaks if used improperly or provide specialized functions not likely to be of interest to most users. Nevertheless, these functions provide a convenient method of performing Geographic Information System (GIS) functions that would be difficult to do with Argus ONE alone.
\end{abstract}

\section{Introduction}

Argus $\mathrm{ONE}^{\mathrm{TM}}$ is a Geographic Information System for numerical modeling developed by Argus Interware. It can serve as a platform for creating graphical userinterfaces for models (for example Voss and others (1997) and Winston (2000)). It can create finite-element meshes and finite-difference grids and assign properties to nodes, elements, or cells based on GIS analyses that it performs. It can write the input files for numerical models and graphically display the results of those models. The functionality of Argus ONE can be extended through the use of Plug-In Extensions (PIEs). A PIE can provide an interface for a particular model, add a command to the Argus ONE menu structure, add a GIS function that can be used by Argus ONE, import data into Argus ONE, export data from Argus ONE, or implement a method of interpolating among data.

This report describes a number of interpolation methods, commands, and functions that have been added to Argus ONE through the use of PIEs. The PIEs described in this report are not specific to any particular type of model but instead may find application under a variety of circumstances. The new interpolation methods are much faster than existing interpolation methods in Argus ONE and can also incorporate anisotropy. The commands listed in tables 1 and 2 are useful for copying, editing, converting, or importing information. The commands listed in table 2 are hidden; special steps must be taken before they appear in 
the menu structure. The functions listed in tables 3 and 4 provide convenient methods for evaluating, converting, or storing information. The functions in table 4 are hidden and do not appear in the Argus ONE Expression editor unless special steps are taken to reveal them but still will be accepted by Argus ONE. The hidden commands and functions were hidden for three reasons. (1) The hidden commands circumvent protections built into Argus ONE against users inadvertently changing or deleting information. (2) Some of the hidden functions can not be used in the Argus ONE parameters because they either may allocate memory that must be released later by a call to another function or because they use data stored in a previous function call. (3) The hidden functions provide specialized capabilities that are unlikely to be useful to most users although they are used extensively in the export template for the MODFLOW GUI (Winston, 2000).

Some of the PIEs described in this report, such as the List and BlockList PIEs, have been used in the MODFLOW GUI (Winston, 2000) or SUTRA GUI (Voss and others, 1997) and are distributed with them. Others have not been released previously.

The purpose for creating these PIEs was to meet needs within the U.S. Geological Survey (USGS). The USGS is using Argus ONE as a graphical-user interface for its groundwater modeling programs. Argus ONE was chosen for this purpose because it has an open architecture that allows the USGS to customize the graphical-user interfaces with less effort than would be required if they were created from scratch. Argus ONE has numerous GIS functions that facilitate the convertion of graphical information to the numerical information required by ground-water modeling programs. In some cases, however, Argus ONE did not have certain capabilities that would be useful to USGS scientists using the graphical-user interfaces. For example, Argus ONE did not have a method for importing data from USGS digital elevation models. To remedy the situation, a PIE command (described in this report) was developed that could read USGS digital elevation models and convert the data into a format that could be used by Argus ONE. Most of the commands and functions described in this report were developed for similar reasons. In a few cases, however, commands or functions described in this report duplicate capabilities already present in Argus ONE. These commands and functions were a byproduct of developing other commands and functions not present in Argus ONE. In some cases, the new functions may be faster than the corresponding functions in Argus ONE. It would have been possible to use such functions only for internal calculations. However, making the functions available as independent functions required almost no effort. In specific cases, the new functions can facilitate better performance of the graphical user interface.

An example of a command that partially duplicates Argus ONE functionality but also adds new functionality is the Edit Grid command. In Argus ONE, the user can set the grid angle by typing a value of the grid angle, but there is no graphical way of specifying the grid angle. With the Edit Grid command, the user can specify the grid angle graphically. In Argus ONE, the user can set the position of an interior grid line by double-clicking on it or by dragging it to a new position. However, Argus ONE does not have a similar capability for the exterior grid lines. With the Edit Grid command, the user can set the positions of both interior and exterior grid lines.

The PIEs described in this report are written in Object Pascal using the Borland Delphi version 5 compiler. When published, the source code will be available at http://water.usgs.gov/nrp/gwsoftware/. 
Table 1. Commands added to Argus ONE in Utility.dII

\begin{tabular}{|c|c|}
\hline Command & Purpose \\
\hline PIEs|Edit|Edit Contours & Editing the exact positions of nodes on contours \\
\hline PIEs|Edit|DeclutterContours & Removing extra nodes from contours \\
\hline PIEs|Edit|Join Contours & $\begin{array}{l}\text { Joining together open contours whose ends match } \\
\text { exactly and which have exactly the same contour values }\end{array}$ \\
\hline PIEs|Edit|Edit Grid & Creating and editing grids \\
\hline PIEs|Edit|Edit Data & $\begin{array}{l}\text { Editing the locations and values of data points on a data } \\
\text { layer }\end{array}$ \\
\hline $\begin{array}{l}\text { PIEs|Edit|Create Parameters in } \\
\text { Multiple Layers }\end{array}$ & Creates identical copies of parameters in multiple layers \\
\hline PIEs|Edit|Set Multiple Parameters & $\begin{array}{l}\text { Sets the value of multiple parameters in multiple layers } \\
\text { in one step }\end{array}$ \\
\hline PIEs|Import|Import Gridded Data & Importing data points at the centers of grid blocks \\
\hline $\begin{array}{l}\text { PIEs|Import|Import Points from } \\
\text { Spreadsheet }\end{array}$ & Importing point contours from a spreadsheet \\
\hline $\begin{array}{l}\text { PIEs|Import|Import Contours } \\
\text { from Spreadsheet }\end{array}$ & $\begin{array}{l}\text { Importing point, open, or closed contours from a } \\
\text { spreadsheet }\end{array}$ \\
\hline PIEs|Import|Sample DEM Data & Import Digital Elevation Models into Argus ONE \\
\hline PIEs|Import|Copy Tri Mesh & $\begin{array}{l}\text { Copying a finite-element mesh with triangular elements } \\
\text { to another mesh layer }\end{array}$ \\
\hline PIEs|Import|Copy Quad Mesh & $\begin{array}{l}\text { Copying a finite-element mesh with quadrilateral } \\
\text { elements to another mesh layer }\end{array}$ \\
\hline PIEs|Convert|Contours To Data & Converting contours to data points on a data layer \\
\hline PIEs|Convert|Data to Contours & Converting data points on a data layer to point contours \\
\hline $\begin{array}{l}\text { PIEs|Convert|Reverse Contours } \\
\text { on Clipboard }\end{array}$ & Reversing the order in which nodes occur in a contour \\
\hline $\begin{array}{l}\text { PIEs|Convert|Mesh Objects To } \\
\text { Contours }\end{array}$ & $\begin{array}{l}\text { Creating contours along the edges of selected finite } \\
\text { elements }\end{array}$ \\
\hline PIEs|Convert|Mesh To Contours & $\begin{array}{l}\text { Creating closed contours that duplicate the shapes of } \\
\text { finite elements }\end{array}$ \\
\hline
\end{tabular}

Table 2. Hidden commands added to Argus ONE in Utility.dll

\begin{tabular}{ll}
\hline \multicolumn{1}{c}{ Command } & \multicolumn{1}{c}{ Purpose } \\
\hline PIEs|Set Parameter Locks & $\begin{array}{l}\text { Hidden command that locks or unlocks multiple parameters } \\
\text { in one step }\end{array}$ \\
PIEs|Delete Multiple Layers & Hidden command that deletes multiple layers in one step \\
\hline
\end{tabular}


Table 3. Functions added to Argus ONE

\begin{tabular}{|c|c|c|}
\hline Function(arguments) & File & Result \\
\hline $\begin{array}{l}\text { Utility_CheckVersion(First_Digit, } \\
\text { Second_Digit, Third_Digit, } \\
\text { Fourth_Digit) }\end{array}$ & Utility.dll & $\begin{array}{l}\text { Returns True if the version } \\
\text { number of the Utility PIE is } \\
\text { greater than or equal to the } \\
\text { version number passed in the } \\
\text { arguments }\end{array}$ \\
\hline $\begin{array}{l}\text { EvalRealAtXY(X,Y, } \\
\text { "Expression_As_Quoted_String", } \\
\text { ["Layer_Name_As_Quoted_String"]) }\end{array}$ & Utility.dll & $\begin{array}{l}\text { A real number based on the } \\
\text { evaluation of } \\
\text { "Expression_As_Quoted_String" } \\
\text { evaluated at }(\mathrm{X}, \overline{\mathrm{Y}})\end{array}$ \\
\hline $\begin{array}{l}\text { EvalIntegerAtXY(X, Y, } \\
\text { "Expression_As_Quoted_String", } \\
\text { ["Layer_Name_As_Quoted_String"]) }\end{array}$ & Utility.dll & $\begin{array}{l}\text { An integer based on the } \\
\text { evaluation of } \\
\text { "Expression_As_Quoted_String" } \\
\text { evaluated at }(\mathrm{X}, \overline{\mathrm{Y}})\end{array}$ \\
\hline $\begin{array}{l}\text { EvalBooleanAtXY(X, Y, } \\
\text { "Expression_As_Quoted_String", } \\
\text { ["Layer_Name_As_Quoted_String"]) }\end{array}$ & Utility.dll & $\begin{array}{l}\text { A Boolean based on the } \\
\text { evaluation of } \\
\text { "Expression_As_Quoted_String" } \\
\text { evaluated at }(\mathrm{X}, \overline{\mathrm{Y}})\end{array}$ \\
\hline $\begin{array}{l}\text { EvalStringAtXY(X, Y, } \\
\text { "Expression_As_Quoted_String", } \\
\text { ["Layer_Name_As_Quoted_String"]) }\end{array}$ & Utility.dll & $\begin{array}{l}\text { A string based on the evaluation } \\
\text { of } \\
\text { "Expression_As_Quoted_String" } \\
\text { evaluated at }(\mathrm{X}, \overline{\mathrm{Y}})\end{array}$ \\
\hline Rotated $\mathrm{X}\left({ }^{\prime} \mathrm{X}\right.$ ', 'Y', 'GridAngle') & Utility.dll & $\begin{array}{l}\text { A row/column position converted } \\
\text { to an } \mathrm{X} \text { coordinate }\end{array}$ \\
\hline Rotated $Y(' X$ ', ' $Y$ ', 'GridAngle') & Utility.dll & $\begin{array}{l}\text { A row/column position converted } \\
\text { to an Y coordinate }\end{array}$ \\
\hline GetMyDirectory() & $\begin{array}{l}\text { GetMyDirectory. } \\
\text { dll }\end{array}$ & $\begin{array}{l}\text { The name of the directory in } \\
\text { which the PIE is installed }\end{array}$ \\
\hline $\begin{array}{l}\text { RF_Get_Value_From_File(Key, } \\
\text { Default_Value, [FileName]) }\end{array}$ & ReadFileValue.dll & $\begin{array}{l}\text { A value from a file associated } \\
\text { with "Key" or if "Key" is not } \\
\text { found, the Default_Value }\end{array}$ \\
\hline RF_Clear_Files () & ReadFileValue.dll & $\begin{array}{l}\text { Attempts to clear a file and } \\
\text { returns True if the function } \\
\text { succeeds }\end{array}$ \\
\hline RF_Save_Files() & ReadFileValue.dll & $\begin{array}{l}\text { Attempts to save a file and } \\
\text { returns True if the function } \\
\text { succeeds }\end{array}$ \\
\hline
\end{tabular}


Table 3 (Continued)

\begin{tabular}{|c|c|c|}
\hline Function(arguments) & File & Result \\
\hline $\begin{array}{l}\text { RF_CheckVersion(First_Digit, } \\
\text { Second_Digit, Third_Digit, } \\
\text { Fourth_Digit) }\end{array}$ & ReadFileValue.dll & $\begin{array}{l}\text { Returns True if the version } \\
\text { number of the ReadFile PIE is } \\
\text { greater than or equal to the } \\
\text { version number passed in the } \\
\text { arguments }\end{array}$ \\
\hline Sec2Day(Number) & Utility.dll & $\begin{array}{l}\text { Converts number from seconds to } \\
\text { days and returns the result }\end{array}$ \\
\hline Day2Sec(Number) & Utility.dll & $\begin{array}{l}\text { Converts Number from days to } \\
\text { seconds and returns the result }\end{array}$ \\
\hline K2F(Number) & Utility.dll & $\begin{array}{l}\text { Converts Number from degrees } \\
\text { Kelvin to degrees Fahrenheit and } \\
\text { returns the result }\end{array}$ \\
\hline F2K(Number) & Utility.dll & $\begin{array}{l}\text { Converts Number from degrees } \\
\text { Fahrenheit to degrees Kelvin and } \\
\text { returns the result }\end{array}$ \\
\hline J2BTU(Number) & Utility.dll & $\begin{array}{l}\text { Converts Number from Joules to } \\
\text { British Thermal Units (BTU) and } \\
\text { returns the result }\end{array}$ \\
\hline BTU2J(Number) & Utility.dll & $\begin{array}{l}\text { Converts Number from BTU to } \\
\text { Joules and returns the result }\end{array}$ \\
\hline sq_m2sq_ft(Number) & Utility.dll & $\begin{array}{l}\text { Converts Number from square } \\
\text { meters to square feet and returns } \\
\text { the result }\end{array}$ \\
\hline sq_ft2sq_m(Number) & Utility.dll & $\begin{array}{l}\text { Converts Number from square } \\
\text { feet to square meters and returns } \\
\text { the result }\end{array}$ \\
\hline cu_m2cu_ft(Number) & Utility.dll & $\begin{array}{l}\text { Converts Number from cubic } \\
\text { meters to cubic feet and returns } \\
\text { the result }\end{array}$ \\
\hline cu_ft2cu_m(Number) & Utility.dll & $\begin{array}{l}\text { Converts Number from cubic feet } \\
\text { to cubic meters and returns the } \\
\text { result }\end{array}$ \\
\hline m_s2ft_day(Number) & Utility.dll & $\begin{array}{l}\text { Converts Number from meter- } \\
\text { seconds to feet-days and returns } \\
\text { the result }\end{array}$ \\
\hline $\mathrm{ft}$ day $2 \mathrm{~m} \_\mathrm{s}($ Number) & Utility.dll & $\begin{array}{l}\text { Converts Number from feet-days } \\
\text { to meter-seconds and returns the } \\
\text { result }\end{array}$ \\
\hline
\end{tabular}


Table 3 (Continued)

\begin{tabular}{|c|c|c|}
\hline Function(arguments) & File & Result \\
\hline Pa2psi(Number) & Utility.dll & $\begin{array}{l}\text { Converts Number from Pascals to } \\
\text { pounds per square inch (psi) and } \\
\text { returns the result }\end{array}$ \\
\hline psi2Pa(Number) & Utility.dll & $\begin{array}{l}\text { Converts Number from psi to } \\
\text { Pascals and returns the result }\end{array}$ \\
\hline m_per_s2ft_per_day(Number) & Utility.dll & $\begin{array}{l}\text { Converts Number from meters } \\
\text { per second to feet per day and } \\
\text { returns the result }\end{array}$ \\
\hline $\mathrm{ft}$ _per_day $2 \mathrm{~m}$ _per_s(Number) & Utility.dll & $\begin{array}{l}\text { Converts Number from feet per } \\
\text { day to meters per second and } \\
\text { returns the result }\end{array}$ \\
\hline sq_m_per_s2sq_ft_per_day(Number) & Utility.dll & $\begin{array}{l}\text { Converts Number from square } \\
\text { meters per second to square feet } \\
\text { per day or from cubic meters per } \\
\text { meter-second to cubic feet per } \\
\text { foot-day and returns the result }\end{array}$ \\
\hline sq_ft_per_day2sq_m_per_s(Number) & Utility.dll & $\begin{array}{l}\text { Converts Number from square } \\
\text { feet per day to square meters per } \\
\text { second and returns the result }\end{array}$ \\
\hline cu_m_per_s2cu_ft_per_day(Number) & Utility.dll & $\begin{array}{l}\text { Converts Number from cubic } \\
\text { meters per second to cubic feet } \\
\text { per day and returns the result }\end{array}$ \\
\hline cu_ft_per_day2cu_m_per_s(Number) & Utility.dll & $\begin{array}{l}\text { Converts Number from cubic feet } \\
\text { per day to cubic meters per } \\
\text { second and returns the result }\end{array}$ \\
\hline I_per_s2cu_ft_per_day(Number) & Utility.dll & $\begin{array}{l}\text { Converts Number from liters per } \\
\text { second to cubic feet per day and } \\
\text { returns the result }\end{array}$ \\
\hline cu_ft_per_day2l_per_s(Number) & Utility.dll & $\begin{array}{l}\text { Converts Number from cubic feet } \\
\text { per day to liters per second and } \\
\text { returns the result }\end{array}$ \\
\hline kg_per_s2lb_per_day(Number) & Utility.dll & $\begin{array}{l}\text { Converts Number from kilograms } \\
\text { per second to pounds per day and } \\
\text { returns the result }\end{array}$ \\
\hline lb_per_day $2 \mathrm{~kg}$ _per_s(Number) & Utility.dll & $\begin{array}{l}\text { Converts Number from pounds } \\
\text { per day to kilograms per second } \\
\text { and returns the result }\end{array}$ \\
\hline Pa_per_s2psi_per_day(Number) & Utility.dll & $\begin{array}{l}\text { Converts Number from Pascals } \\
\text { per second to psi per day and } \\
\text { returns the result }\end{array}$ \\
\hline
\end{tabular}


Table 3 (Continued)

\begin{tabular}{|c|c|c|}
\hline Function(arguments) & File & Result \\
\hline psi_per_day2Pa_per_s(Number) & Utility.dll & $\begin{array}{l}\text { Converts Number from psi per } \\
\text { day to Pascals per second and } \\
\text { returns the result }\end{array}$ \\
\hline kg_per_cu_m2lb_per_cu_ft(Number) & Utility.dll & $\begin{array}{l}\text { Converts Number from kilograms } \\
\text { per cubic meter to pounds per } \\
\text { cubic foot and returns the result }\end{array}$ \\
\hline lb_per_cu_ft $2 \mathrm{~kg} \_p e r \_c u \_m$ (Number) & Utility.dll & $\begin{array}{l}\text { Converts Number from pounds } \\
\text { per cubic foot to kilograms per } \\
\text { cubic meter and returns the result }\end{array}$ \\
\hline $\begin{array}{l}\text { W_per_cu_m2BTU_per_hr_cu_ft } \\
\text { (Number) }\end{array}$ & Utility.dll & $\begin{array}{l}\text { Converts Number from Watts per } \\
\text { cubic meter to BTU per hour- } \\
\text { cubic foot and returns the result }\end{array}$ \\
\hline $\begin{array}{l}\text { BTU_per_hr_cu_ft } 2 \mathrm{~W} \text { _per_cu_m } \\
\text { (Number) }\end{array}$ & Utility.dll & $\begin{array}{l}\text { Converts Number from BTU per } \\
\text { hour-cubic foot to Watts per } \\
\text { cubic meter and returns the result }\end{array}$ \\
\hline J_per_kg2BTU_per_lb(Number) & Utility.dll & $\begin{array}{l}\text { Converts Number from Joules per } \\
\text { kilogram to BTU per pound and } \\
\text { returns the result }\end{array}$ \\
\hline BTU_per_lb2J_per_kg(Number) & Utility.dll & $\begin{array}{l}\text { Converts Number from BTU per } \\
\text { pound to Joules per kilogram and } \\
\text { returns the result }\end{array}$ \\
\hline J_per_kg2ft_lb_f_per_lb_m(Number) & Utility.dll & $\begin{array}{l}\text { Converts Number from Joules per } \\
\text { kilogram to foot-pound force per } \\
\text { pound mass and returns the result }\end{array}$ \\
\hline $\mathrm{ft} \_\mathrm{lb}$ f_per_lb_m2J_per_kg(Number) & Utility.dll & $\begin{array}{l}\text { Converts Number from foot- } \\
\text { pound force per pound mass to } \\
\text { Joules per kilogram and returns } \\
\text { the result }\end{array}$ \\
\hline cu_m_per_kg2cu_ft_per_lb(Number) & Utility.dll & $\begin{array}{l}\text { Converts Number from cubic } \\
\text { meter per kilogram to cubic foot } \\
\text { per pound and returns the result }\end{array}$ \\
\hline cu_ft_per_lb2cu_m_per_kg(Number) & Utility.dll & $\begin{array}{l}\text { Converts Number from cubic foot } \\
\text { per pound to cubic meter per } \\
\text { kilogram and returns the result }\end{array}$ \\
\hline $\begin{array}{l}\text { cu_m_per_sq_m_s2cu_ft_per_sq_ft_ } \\
\text { day (Number) }\end{array}$ & Utility.dll & $\begin{array}{l}\text { Converts Number from cubic } \\
\text { meter per square meter-second to } \\
\text { cubic foot per square foot-day } \\
\text { and returns the result }\end{array}$ \\
\hline
\end{tabular}


Table 3 (Continued)

\begin{tabular}{|c|c|c|}
\hline Function(arguments) & File & Result \\
\hline $\begin{array}{l}\text { cu_ft_per_sq_ft_day2cu_m_per_sq_ } \\
\text { m_s (Number) }\end{array}$ & Utility.dll & $\begin{array}{l}\text { Converts Number from cubic foot } \\
\text { per square foot-day to cubic } \\
\text { meter per square meter-second } \\
\text { and returns the result }\end{array}$ \\
\hline $\begin{array}{l}\text { W_per_sq_m2BTU_per_hr_sq_ft } \\
\text { (Number) }\end{array}$ & Utility.dll & $\begin{array}{l}\text { Converts Number from Watts per } \\
\text { square meter to BTU per hour- } \\
\text { square foot and returns the result }\end{array}$ \\
\hline $\begin{array}{l}\text { BTU_per_hr_sq_ft2W_per_sq_m } \\
\text { (Number) }\end{array}$ & Utility.dll & $\begin{array}{l}\text { Converts Number from BTU per } \\
\text { hour-square foot to Watts per } \\
\text { square meter and returns the } \\
\text { result }\end{array}$ \\
\hline $\begin{array}{l}\text { kg_per_sq_m_s2lb_per_sq_ft_day } \\
\text { (Number) }\end{array}$ & Utility.dll & $\begin{array}{l}\text { Converts Number from kilogram } \\
\text { per square meter-second to pound } \\
\text { per square foot-day and returns } \\
\text { the result }\end{array}$ \\
\hline $\begin{array}{l}\text { lb_per_sq_ft_day2kg_per_sq_m_s } \\
\text { (Number) }\end{array}$ & Utility.dll & $\begin{array}{l}\text { Converts Number from pound per } \\
\text { square foot-day to kilogram per } \\
\text { square meter-second and returns } \\
\text { the result }\end{array}$ \\
\hline kg_per_m_s2cP(Number) & Utility.dll & $\begin{array}{l}\text { Converts Number from kilogram } \\
\text { per meter-second to centipoises } \\
\text { and returns the result }\end{array}$ \\
\hline cP2kg_per_m_s(Number) & Utility.dll & $\begin{array}{l}\text { Converts Number from } \\
\text { centipoises to kilogram per } \\
\text { meter-second and returns the } \\
\text { result }\end{array}$ \\
\hline $\begin{array}{l}\text { J_per_kg_m2BTU_per_lb_ft } \\
\text { (Number) }\end{array}$ & Utility.dll & $\begin{array}{l}\text { Converts Number from Joules per } \\
\text { kilogram-meter to BTU per } \\
\text { pound-foot and returns the result }\end{array}$ \\
\hline $\begin{array}{l}\text { BTU_per_lb_ft2J_per_kg_m } \\
\text { (Number) }\end{array}$ & Utility.dll & $\begin{array}{l}\text { Converts Number from BTU per } \\
\text { pound-foot to Joules per } \\
\text { kilogram-meter and returns the } \\
\text { result }\end{array}$ \\
\hline $\begin{array}{l}\text { W_per_m_deg_C2BTU_per_ft_hr_ } \\
\text { deg_F (Number) }\end{array}$ & Utility.dll & $\begin{array}{l}\text { Converts Number from Watts per } \\
\text { meter-degree Celsius to BTU per } \\
\text { foot-hour-degree Fahrenheit and } \\
\text { returns the result }\end{array}$ \\
\hline $\begin{array}{l}\text { BTU_per_ft_hr_deg_F2W_per_m } \\
\text { deg_C (Number) }\end{array}$ & Utility.dll & $\begin{array}{l}\text { Converts Number from BTU per } \\
\text { foot-hour-degree Fahrenheit to } \\
\text { Watts per meter-degree Celsius } \\
\text { and returns the result }\end{array}$ \\
\hline
\end{tabular}


Table 3 (Continued)

\begin{tabular}{|c|c|c|}
\hline $\begin{array}{r}\text { Function } \\
\end{array}$ & File & Result \\
\hline $\begin{array}{l}\text { W_per_sq_m_deg_C2BTU_per_hr_ } \\
\text { sq_ft_deg_F (Number) }\end{array}$ & Utility.dll & $\begin{array}{l}\text { Converts Number from Watts per } \\
\text { square meter-degree Celsius to } \\
\text { BTU per square foot-hour-degree } \\
\text { Fahrenheit and returns the result }\end{array}$ \\
\hline $\begin{array}{l}\text { BTU_per_hr_sq_ft_deg_F2W_per_sq } \\
\text { _m_deg_C (Number) }\end{array}$ & Utility.dll & $\begin{array}{l}\text { Converts Number from BTU per } \\
\text { square foot-hour-degree } \\
\text { Fahrenheit to Watts per square } \\
\text { meter-degree Celsius and returns } \\
\text { the result }\end{array}$ \\
\hline $\begin{array}{l}\text { J_per_kg_deg_C2BTU_per_lb_deg } \\
\text { F (Number) }\end{array}$ & Utility.dll & $\begin{array}{l}\text { Converts Number from Joules per } \\
\text { kilogram-degree Celsius to BTU } \\
\text { per pound-degree Fahrenheit and } \\
\text { returns the result }\end{array}$ \\
\hline $\begin{array}{l}\text { BTU_per_lb_deg_F2J_per_kg_deg } \\
\text { C (Number) }\end{array}$ & Utility.dll & $\begin{array}{l}\text { Converts Number from BTU per } \\
\text { pound-degree Fahrenheit to } \\
\text { Joules per kilogram-degree } \\
\text { Celsius and returns the result }\end{array}$ \\
\hline $\begin{array}{l}\text { J_per_cu m_deg_C2BTU_per_cu_ft } \\
\text { _deg_F (Number) }\end{array}$ & Utility.dll & $\begin{array}{l}\text { Converts Number from Joules per } \\
\text { cubic meter-degree Celsius to } \\
\text { BTU per cubic foot-degree } \\
\text { Fahrenheit and returns the result }\end{array}$ \\
\hline $\begin{array}{l}\text { BTU_per_cu_ft_deg_F2J_per_cu_m } \\
\text { deg_C (Number) }\end{array}$ & Utility.dll & $\begin{array}{l}\text { Converts Number from BTU per } \\
\text { cubic foot -degree Fahrenheit to } \\
\text { Joules per cubic meter-degree } \\
\text { Celsius and returns the result }\end{array}$ \\
\hline $\begin{array}{l}\text { cu_m_per_s_m_Pa2cu_ft_per_day_lb } \\
\text { sq__in (Number) }\end{array}$ & Utility.dll & $\begin{array}{l}\text { Converts Number from cubic } \\
\text { meter per second-meter-Pascal to } \\
\text { cubic foot per day-point-square } \\
\text { inch and returns the result }\end{array}$ \\
\hline $\begin{array}{l}\mathrm{cu} \text { _ft_per_day_lb_sq_in } 2 \mathrm{cu} \text { _m_per_s } \\
\text { _m_Pa (Number) }\end{array}$ & Utility.dll & $\begin{array}{l}\text { Converts Number from cubic foot } \\
\text { per day-point-square inch to } \\
\text { cubic meter per second-meter- } \\
\text { Pascal and returns the result }\end{array}$ \\
\hline $\begin{array}{l}\text { m_per_sq_sec2ft_per_sq_day } \\
\text { (Number) }\end{array}$ & Utility.dll & $\begin{array}{l}\text { Converts Number from meter per } \\
\text { square second to foot per square } \\
\text { day-point-square inch and returns } \\
\text { the result }\end{array}$ \\
\hline $\begin{array}{l}\mathrm{ft} \text { _per_sq_day } 2 \mathrm{~m} \text { _per_sq_sec } \\
\text { (Number) }\end{array}$ & Utility.dll & $\begin{array}{l}\text { Converts Number from foot per } \\
\text { square day to meter per square } \\
\text { second and returns the result }\end{array}$ \\
\hline
\end{tabular}


Table 4. Hidden functions added to Argus ONE

\begin{tabular}{|c|c|c|}
\hline Function(arguments) & File & Result \\
\hline IsOK(Message, [HideCancel]) & OkCancel.dll & $\begin{array}{l}\text { Displays a dialog box with a } \\
\text { message and several buttons } \\
\text { Returns True or False depending on } \\
\text { which button the user pushes }\end{array}$ \\
\hline $\begin{array}{l}\text { ok_Add_Radio_Choice(Message, } \\
\text { [Message], [Message], [Message], } \\
\text { [Message]) }\end{array}$ & OkCancel.dll & $\begin{array}{l}\text { Adds up to five radio button to a } \\
\text { dialog box and returns True if it } \\
\text { succeeds }\end{array}$ \\
\hline $\begin{array}{l}\text { ok_Get_Radio_Choice(Message, } \\
\text { [Choices_Height], [Width], } \\
\text { [Question_Height]) }\end{array}$ & OkCancel.dll & $\begin{array}{l}\text { Returns the index of the radio } \\
\text { button that the user selects with the } \\
\text { first radio button given an index of } \\
\text { zero }\end{array}$ \\
\hline ok_Radio_Free() & OkCancel.dll & $\begin{array}{l}\text { Returns True if the dialog box with } \\
\text { the radio buttons has been } \\
\text { destroyed }\end{array}$ \\
\hline $\begin{array}{l}\text { ok_UserFloat(Message, Response, } \\
\text { [Minimum], [Maximum]) }\end{array}$ & OkCancel.dll & $\begin{array}{l}\text { Displays a dialog box on which a } \\
\text { user may enter a real number, the } \\
\text { result is the real number that the } \\
\text { user enters }\end{array}$ \\
\hline $\begin{array}{l}\text { ok_UserInteger(Message, Response, } \\
\text { [Minimum], [Maximum]) }\end{array}$ & OkCancel.dll & $\begin{array}{l}\text { Displays a dialog box on which a } \\
\text { user may enter an integer, the result } \\
\text { is the integer that the user enters }\end{array}$ \\
\hline $\begin{array}{l}\text { ok_CheckVersion(First_Digit, } \\
\text { Second_Digit, Third_Digit, } \\
\text { Fourth_Digit) }\end{array}$ & OkCancel.dll & $\begin{array}{l}\text { Returns True if the version number } \\
\text { of the OkCancle PIE is greater than } \\
\text { or equal to the version number } \\
\text { passed in the arguments }\end{array}$ \\
\hline $\begin{array}{l}\text { ProgressBarInitialize(Number, } \\
\text { [Show_Cancel]) }\end{array}$ & ProgressBar.dll & $\begin{array}{l}\text { Attempts to create a dialog box } \\
\text { with a progress bar and returns } \\
\text { True if it succeeds }\end{array}$ \\
\hline ProgressBarFree () & ProgressBar.dll & $\begin{array}{l}\text { Attempts to destroy the dialog box } \\
\text { with the progress bar and returns } \\
\text { True if it succeeds }\end{array}$ \\
\hline ProgressBarMax(Number) & ProgressBar.dll & $\begin{array}{l}\text { Attempts to set the maximum value } \\
\text { of the progress bar and returns True } \\
\text { if the Abort button has not been } \\
\text { pressed and the function succeeds }\end{array}$ \\
\hline ProgressBarAdvance() & ProgressBar.dll & $\begin{array}{l}\text { Attempts to advance the progress } \\
\text { bar by one and returns True if the } \\
\text { Abort button has not been pressed } \\
\text { and the function succeeds }\end{array}$ \\
\hline
\end{tabular}


Table 4 (Continued)

\begin{tabular}{|c|c|c|}
\hline Function(arguments) & File & Result \\
\hline ProgressBarSetMessage(Message) & ProgressBar.dll & $\begin{array}{l}\text { Attempts to display a message with } \\
\text { the progress bar and returns True if } \\
\text { the Abort button has not been } \\
\text { pressed and the function succeeds }\end{array}$ \\
\hline ProgressBarAddLine(Message) & ProgressBar.dll & $\begin{array}{l}\text { Attempts to add a line to the memo- } \\
\text { box beneath the progress bar and } \\
\text { returns True if the Abort button has } \\
\text { not been pressed and the function } \\
\text { succeeds }\end{array}$ \\
\hline ProgressBarSaveToFile(File_Name) & ProgressBar.dll & $\begin{array}{l}\text { Attempts to save the lines in the } \\
\text { memo box and returns the number } \\
\text { of lines in the file it saves }\end{array}$ \\
\hline $\begin{array}{l}\text { ProgressBarCheckVersion(First_Digit, } \\
\text { Second_Digit, Third_Digit, } \\
\text { Fourth_Digit) }\end{array}$ & ProgressBar.dll & $\begin{array}{l}\text { Returns True if the version number } \\
\text { of the ProgressBar PIE is greater } \\
\text { than or equal to the version number } \\
\text { passed in the arguments }\end{array}$ \\
\hline $\begin{array}{l}\text { Join_Files(First_File, Second_File, } \\
\text { Result_File) }\end{array}$ & JoinFiles.dll & $\begin{array}{l}\text { Attempts to concatenate two files } \\
\text { and returns True if the function } \\
\text { succeeds }\end{array}$ \\
\hline Delete_File(File_Name) & JoinFiles.dll & $\begin{array}{l}\text { Attempts to delete a file and returns } \\
\text { True if the function succeeds }\end{array}$ \\
\hline $\begin{array}{l}\text { Rename_File(Old_File_Name, } \\
\text { New_File_Name) }\end{array}$ & JoinFiles.dll & $\begin{array}{l}\text { Attempts to rename a file and } \\
\text { returns True if the function } \\
\text { succeeds }\end{array}$ \\
\hline $\begin{array}{l}\text { Split_File('Input_File, First_File, } \\
\text { Search_String, Second_File [, } \\
\text { Search_String, Third_File] [, } \\
\text { Search_String, Fourth_File]...[, } \\
\text { Search_String, Thirtieth_File]) }\end{array}$ & JoinFiles.dll & $\begin{array}{l}\text { Attempts to split a file into } 2 \text { to } 30 \\
\text { separate files and returns True if the } \\
\text { function succeeds }\end{array}$ \\
\hline Int2Str(Number) & JoinFiles.dll & $\begin{array}{l}\text { Returns the base } 36 \text { representation } \\
\text { of Number }\end{array}$ \\
\hline $\begin{array}{l}\text { JF_CopyLines(Old_File_Name, } \\
\text { New_File_Name, Line_Count, } \\
\text { [Is_Local_File]) }\end{array}$ & JoinFiles.dll & $\begin{array}{l}\text { Copies Line_Count from } \\
\text { Old_File_Name to a new file } \\
\text { named New_File_Name and returns } \\
\text { True if it succeeds. }\end{array}$ \\
\hline $\begin{array}{l}\text { JF_CheckVersion(First_Digit, } \\
\text { Second_Digit, Third_Digit, } \\
\text { Fourth_Digit) }\end{array}$ & JoinFiles.dll & $\begin{array}{l}\text { Returns True if the version number } \\
\text { of the JoinFile PIE is greater than } \\
\text { or equal to the version number } \\
\text { passed in the arguments }\end{array}$ \\
\hline L_Initialize() & List.dll & True (obsolete function) \\
\hline
\end{tabular}


Table 4 (Continued)

\begin{tabular}{|c|c|c|}
\hline Function(arguments) & File & Result \\
\hline L_CreateNewList() & List.dll & $\begin{array}{l}\text { Creates a list and returns the } \\
\text { number of the list that was created }\end{array}$ \\
\hline L_SetListSize(ListIndex, Size) & List.dll & $\begin{array}{l}\text { Sets the size of a list and returns } \\
\text { True if the function succeeds }\end{array}$ \\
\hline L_GetListSize(ListIndex) & List.dll & $\begin{array}{l}\text { Returns the number of items in the } \\
\text { list }\end{array}$ \\
\hline L_FreeAList(ListIndex) & List.dll & $\begin{array}{l}\text { Attempts to remove all items from } \\
\text { a list and returns True if the } \\
\text { function succeeds }\end{array}$ \\
\hline L_AddToList(ListIndex, Value) & List.dll & $\begin{array}{l}\text { Adds a value to a list and returns } \\
\text { the position of Value in the list if } \\
\text { the function succeeds }\end{array}$ \\
\hline L_GetFromList(ListIndex, Index) & List.dll & $\begin{array}{l}\text { Returns the item indicated by } \\
\text { ListIndex and Index }\end{array}$ \\
\hline L_SetListItem(ListIndex, Index, Value) & List.dll & $\begin{array}{l}\text { Attempts to set the value of an item } \\
\text { in a list and returns True if the } \\
\text { function succeeds }\end{array}$ \\
\hline L_DeleteListItem(ListIndex, Index) & List.dll & $\begin{array}{l}\text { Attempts to remove an item from a } \\
\text { list and returns True if the function } \\
\text { succeeds }\end{array}$ \\
\hline L_SortList(ListIndex) & List.dll & $\begin{array}{l}\text { Sorts a list in ascending order and } \\
\text { returns True if the function } \\
\text { succeeds }\end{array}$ \\
\hline L_EliminateDuplicates(ListIndex) & List.dll & $\begin{array}{l}\text { Eliminates duplicate values from a } \\
\text { sorted list and returns True if the } \\
\text { function succeeds }\end{array}$ \\
\hline L_IndexOf(ListIndex, Value) & List.dll & $\begin{array}{l}\text { Returns the position of Value in the } \\
\text { sorted list of the position of the first } \\
\text { copy of highest number less than } \\
\text { Value in the sorted list if Value is } \\
\text { not in the sorted list }\end{array}$ \\
\hline L_UnsortedIndexOf(ListIndex, Value) & List.dll & $\begin{array}{l}\text { Returns the position of the first } \\
\text { copy of Value in the list Returns }-1 \\
\text { if Value is not in the list }\end{array}$ \\
\hline $\begin{array}{l}\text { L_CreateNew3DList(Maximum_X, } \\
\text { Maximum_Y, Maximum_Z) }\end{array}$ & List.dll & $\begin{array}{l}\text { Creates a } 3 D \text { list and returns the } \\
\text { number of the } 3 D \text { list that was } \\
\text { created }\end{array}$ \\
\hline L_FreeA3DList(ListIndex) & List.dll & $\begin{array}{l}\text { Attempts to destroy a 3D list and } \\
\text { returns True if the function } \\
\text { succeeds }\end{array}$ \\
\hline
\end{tabular}


Table 4 (Continued)

\begin{tabular}{|c|c|c|}
\hline Function(arguments) & File & Result \\
\hline $\begin{array}{l}\text { L_GetFrom3DList(Listlndex, X_Index, } \\
\text { Y_Index, Z_Index) }\end{array}$ & List.dll & $\begin{array}{l}\text { Returns the item indicated by } \\
\text { ListIndex X_Index, Y_Index, and } \\
\text { Z_Index }\end{array}$ \\
\hline $\begin{array}{l}\text { L_GetFromOneBased3DList(ListIndex, } \\
\text { X_Index, Y_Index, Z_Index) }\end{array}$ & List.dll & $\begin{array}{l}\text { Returns the item indicated by } \\
\text { ListIndex X_Index, Y_Index, and } \\
\text { Z_Index }\end{array}$ \\
\hline $\begin{array}{l}\text { L_Set3DListItem(ListIndex, X_Index, } \\
\text { Y_Index, Z_Index, Value) }\end{array}$ & List.dll & $\begin{array}{l}\text { Sets the value indicated by } \\
\text { ListIndex X_Index, Y_Index, and } \\
\text { Z_Index and returns True if the } \\
\text { function succeeds }\end{array}$ \\
\hline $\begin{array}{l}\text { L_SetOneBased3DListItem(ListIndex, } \\
\text { X_Index, Y_Index, Z_Index, Value) }\end{array}$ & List.dll & $\begin{array}{l}\text { Sets the value indicated by } \\
\text { ListIndex X Index, Y Index, and } \\
Z \text { Z_Index and returns True if the } \\
\text { function succeeds }\end{array}$ \\
\hline L_ResetA3DList(ListIndex) & List.dll & $\begin{array}{l}\text { Sets all members of the } 3 \mathrm{D} \text { list } \\
\text { indicated by ListIndex to } 0 \text { and } \\
\text { returns True if the function } \\
\text { succeeds }\end{array}$ \\
\hline $\begin{array}{l}\text { L_Add3DLists(FirstListIndex, } \\
\text { SecondListIndex, ResultListIndex) }\end{array}$ & List.dll & $\begin{array}{l}\text { Adds the corresponding members } \\
\text { of two } 3 \mathrm{D} \text { lists and returns True if } \\
\text { the function succeeds }\end{array}$ \\
\hline $\begin{array}{l}\text { L_Subtract3DLists(FirstListIndex, } \\
\text { SecondListIndex, ResultListIndex) }\end{array}$ & List.dll & $\begin{array}{l}\text { Subtracts the corresponding } \\
\text { members of one } 3 \mathrm{D} \text { list from those } \\
\text { of another } 3 \mathrm{D} \text { list and returns True } \\
\text { if the function succeeds }\end{array}$ \\
\hline $\begin{array}{l}\text { L_Multiply3DLists(FirstListIndex, } \\
\text { SecondListIndex, ResultListIndex) }\end{array}$ & List.dll & $\begin{array}{l}\text { Multiplies the corresponding } \\
\text { members of two } 3 \mathrm{D} \text { lists and } \\
\text { returns True if the function } \\
\text { succeeds }\end{array}$ \\
\hline $\begin{array}{l}\text { L_Divide3DLists(FirstListlndex, } \\
\text { SecondListIndex, ResultListIndex) }\end{array}$ & List.dll & $\begin{array}{l}\text { Divides the corresponding members } \\
\text { of one } 3 \mathrm{D} \text { list by those of another } \\
\text { 3D list and returns True if the } \\
\text { function succeeds }\end{array}$ \\
\hline $\begin{array}{l}\text { L_Multipy3DByConstant(ListIndex, } \\
\text { ResultListIndex, Value) }\end{array}$ & List.dll & $\begin{array}{l}\text { Multiplies the members of a } 3 D \text { list } \\
\text { by a constant and returns True if the } \\
\text { function succeeds }\end{array}$ \\
\hline $\begin{array}{l}\text { L_Invert3DListMembers(ListIndex, } \\
\text { ResultListIndex) }\end{array}$ & List.dll & $\begin{array}{l}\text { Inverts the members of a 3D list } \\
\text { and returns True if the function } \\
\text { succeeds }\end{array}$ \\
\hline
\end{tabular}


Table 4 (Continued)

\begin{tabular}{|c|c|c|}
\hline Function(arguments) & File & Result \\
\hline L_IsSingPrecUniform(ListIndex) & List.dll & $\begin{array}{l}\text { Returns True if all the members of } \\
\text { a list are the same after being } \\
\text { converted to single precision }\end{array}$ \\
\hline L_GetErrorCount() & List.dll & Returns the number of errors \\
\hline L_FreeAllLists() & List.dll & $\begin{array}{l}\text { Attempts to destroy all lists and 3D } \\
\text { lists and returns True if the function } \\
\text { succeeds }\end{array}$ \\
\hline $\begin{array}{l}\text { L_CheckVersion(First_Digit, } \\
\text { Second_Digit, Third_Digit, } \\
\text { Fourth_Digit) }\end{array}$ & List.dll & $\begin{array}{l}\text { Returns True if the version number } \\
\text { of the List PIE is greater than or } \\
\text { equal to the version number passed } \\
\text { in the arguments }\end{array}$ \\
\hline $\begin{array}{l}\text { BL_InitializeGridInformation } \\
\text { (Grid_Layer_Name_as_String, } \\
\text { [GridType]) }\end{array}$ & BlockList.dll & $\begin{array}{l}\text { Attempts to read grid information } \\
\text { from Argus ONE and returns } 1 \text { if } \\
\text { the function succeeds }\end{array}$ \\
\hline $\begin{array}{l}\text { BL_AddVertexLayer } \\
\text { (Information_Layer_Name_as_String) }\end{array}$ & BlockList.dll & $\begin{array}{l}\text { Attempts to read contour } \\
\text { information from Argus ONE and } \\
\text { returns True if the function } \\
\text { succeeds }\end{array}$ \\
\hline BL_ReInitializeVertexList() & BlockList.dll & $\begin{array}{l}\text { Attempts to delete all contour } \\
\text { information that has been read from } \\
\text { Argus ONE and returns True if the } \\
\text { function succeeds }\end{array}$ \\
\hline BL_GetCountOfCellLists() & BlockList.dll & $\begin{array}{l}\text { Returns the number of lists of cells } \\
\text { currently in memory }\end{array}$ \\
\hline BL_GetCountOfACellList(ListIndex) & BlockList.dll & $\begin{array}{l}\text { Returns the number of cells in a list } \\
\text { of cells }\end{array}$ \\
\hline BL_GetCellRow(ListIndex, Index) & BlockList.dll & Returns the row number of a cell \\
\hline BL_GetCellColumn(Listlndex, Index) & BlockList.dll & $\begin{array}{l}\text { Returns the column number of a } \\
\text { cell }\end{array}$ \\
\hline $\begin{array}{l}\text { BL_GetVertexCount(ListIndex, } \\
\text { CellIndex) }\end{array}$ & BlockList.dll & $\begin{array}{l}\text { Returns the number of vertices of a } \\
\text { contour in a cell }\end{array}$ \\
\hline $\begin{array}{l}\text { BL_GetVertexXPos(Listlndex, } \\
\text { CellIndex, VertexIndex) }\end{array}$ & BlockList.dll & $\begin{array}{l}\text { Returns the X-coordinate of a } \\
\text { vertex }\end{array}$ \\
\hline $\begin{array}{l}\text { BL_GetVertexYPos(ListIndex, } \\
\text { CellIndex, VertexIndex) }\end{array}$ & BlockList.dll & $\begin{array}{l}\text { Returns the Y-coordinate of a } \\
\text { vertex }\end{array}$ \\
\hline $\begin{array}{l}\text { BL_SegmentCount(ListIndex, } \\
\text { CellIndex) }\end{array}$ & BlockList.dll & $\begin{array}{l}\text { Returns the number of segments of } \\
\text { a contour in a cell }\end{array}$ \\
\hline $\begin{array}{l}\text { BL_SegmentFirstX(ListIndex, } \\
\text { CellIndex, SegmentIndex) }\end{array}$ & BlockList.dll & $\begin{array}{l}\text { Returns the X-coordinate of the } \\
\text { first vertex of a segment }\end{array}$ \\
\hline
\end{tabular}


Table 4 (Continued)

\begin{tabular}{|c|c|c|}
\hline Function(arguments) & File & Result \\
\hline $\begin{array}{l}\text { BL_SegmentFirstY(ListIndex, } \\
\text { CellIndex, SegmentIndex) }\end{array}$ & BlockList.dll & $\begin{array}{l}\text { Returns the Y-coordinate of the } \\
\text { first vertex of a segment }\end{array}$ \\
\hline $\begin{array}{l}\text { BL_SegmentSecondX(ListIndex, } \\
\text { Celllndex, SegmentIndex) }\end{array}$ & BlockList.dll & $\begin{array}{l}\text { Returns the X-coordinate of the } \\
\text { second vertex of a segment }\end{array}$ \\
\hline $\begin{array}{l}\text { BL_SegmentSecondY(ListIndex, } \\
\text { CellIndex, SegmentIndex) }\end{array}$ & BlockList.dll & $\begin{array}{l}\text { Returns the Y-coordinate of the } \\
\text { second vertex of a segment }\end{array}$ \\
\hline $\begin{array}{l}\text { BL_SegmentLengthX(ListIndex, } \\
\text { CellIndex, SegmentIndex) }\end{array}$ & BlockList.dll & $\begin{array}{l}\text { Returns the length of a segment in } \\
\text { the X-direction }\end{array}$ \\
\hline $\begin{array}{l}\text { BL_SegmentLengthY(ListIndex, } \\
\text { CellIndex, SegmentIndex) }\end{array}$ & BlockList.dll & $\begin{array}{l}\text { Returns the length of a segment in } \\
\text { the Y-direction }\end{array}$ \\
\hline $\begin{array}{l}\text { BL_SegmentLength(ListIndex, } \\
\text { Celllndex, SegmentIndex) }\end{array}$ & BlockList.dll & Returns the length of a segment \\
\hline $\begin{array}{l}\text { BL_SumSegmentsX(ListIndex, } \\
\text { CellIndex) }\end{array}$ & BlockList.dll & $\begin{array}{l}\text { Returns the sum of the lengths in } \\
\text { the X-direction of all the segments } \\
\text { in a cell }\end{array}$ \\
\hline $\begin{array}{l}\text { BL_SumSegmentsY(ListIndex, } \\
\text { CellIndex) }\end{array}$ & BlockList.dll & $\begin{array}{l}\text { Returns the sum of the lengths in } \\
\text { the Y-direction of all the segments } \\
\text { in a cell }\end{array}$ \\
\hline $\begin{array}{l}\text { BL_SumSegmentLengths(ListIndex, } \\
\text { Celllndex) }\end{array}$ & BlockList.dll & $\begin{array}{l}\text { Returns the sum of the lengths of } \\
\text { all the segments in a cell }\end{array}$ \\
\hline BL_GetCountOfCombinedCellList() & BlockList.dll & $\begin{array}{l}\text { Returns the number of cells } \\
\text { intersected by any contour }\end{array}$ \\
\hline $\begin{array}{l}\text { BL_GetCellRowFromCombinedList } \\
\text { (CellIndex) }\end{array}$ & BlockList.dll & $\begin{array}{l}\text { Returns the row number of a cell } \\
\text { intersected by any contour }\end{array}$ \\
\hline $\begin{array}{l}\text { BL_GetCellColumnFromCombinedList } \\
\text { (CellIndex) }\end{array}$ & BlockList.dll & $\begin{array}{l}\text { Returns the column number of a } \\
\text { cell intersected by any contour }\end{array}$ \\
\hline BL_GetCountOfCrossRowLists() & BlockList.dll & $\begin{array}{l}\text { Returns the number of lists of cells } \\
\text { in which a contour crosses the Y- } \\
\text { coordinate of the cell node }\end{array}$ \\
\hline BL_GetCountOfCrossColumnLists() & BlockList.dll & $\begin{array}{l}\text { Returns the number of cells in a list } \\
\text { of cells in which a contour crosses } \\
\text { the X-coordinate of the cell node }\end{array}$ \\
\hline $\begin{array}{l}\text { BL_GetCountOfACrossRowList } \\
\text { (ListIndex) }\end{array}$ & BlockList.dll & $\begin{array}{l}\text { Returns the number of cells in a list } \\
\text { of cells in which a contour crosses } \\
\text { the Y-coordinate of the cell node }\end{array}$ \\
\hline $\begin{array}{l}\text { BL_GetCountOfACrossColumnList } \\
\text { (ListIndex) }\end{array}$ & BlockList.dll & $\begin{array}{l}\text { Returns the number of cells in a list } \\
\text { of cells in which a contour crosses } \\
\text { the X-coordinate of the cell node }\end{array}$ \\
\hline
\end{tabular}


Table 4 (Continued)

\begin{tabular}{|c|c|c|}
\hline Function(arguments) & File & Result \\
\hline $\begin{array}{l}\text { BL_GetCrossRowRow(ListIndex, } \\
\text { CellIndex) }\end{array}$ & BlockList.dll & $\begin{array}{l}\text { Returns the row number of a cell in } \\
\text { a list of cells in which a contour } \\
\text { crosses the Y-coordinate of the cell } \\
\text { node }\end{array}$ \\
\hline $\begin{array}{l}\text { BL_GetCrossColumnRow(ListIndex, } \\
\text { CellIndex) }\end{array}$ & BlockList.dll & $\begin{array}{l}\text { Returns the row number of a cell in } \\
\text { a list of cells in which a contour } \\
\text { crosses the X-coordinate of the cell } \\
\text { node }\end{array}$ \\
\hline $\begin{array}{l}\text { BL_GetCrossRowColumn(ListIndex, } \\
\text { CellIndex) }\end{array}$ & BlockList.dll & $\begin{array}{l}\text { Returns the column number of a } \\
\text { cell in a list of cells in which a } \\
\text { contour crosses the Y-coordinate of } \\
\text { the cell node }\end{array}$ \\
\hline $\begin{array}{l}\text { BL_GetCrossColumnColumn } \\
\text { (ListIndex, CellIndex) }\end{array}$ & BlockList.dll & $\begin{array}{l}\text { Returns the column number of a } \\
\text { cell in a list of cells in which a } \\
\text { contour crosses the X-coordinate of } \\
\text { the cell node }\end{array}$ \\
\hline $\begin{array}{l}\text { BL_GetCrossRowNeighborColumn } \\
\text { (ListIndex, CellIndex) }\end{array}$ & BlockList.dll & $\begin{array}{l}\text { Returns the column number of the } \\
\text { neighbor of a cell in a list of cells in } \\
\text { which a contour crosses the Y- } \\
\text { coordinate of the cell node }\end{array}$ \\
\hline $\begin{array}{l}\text { BL_GetCrossColumnNeighborRow } \\
\text { (ListIndex, CellIndex) }\end{array}$ & BlockList.dll & $\begin{array}{l}\text { Returns the row number of the } \\
\text { neighbor of a cell in a list of cells in } \\
\text { which a contour crosses the X- } \\
\text { coordinate of the cell node }\end{array}$ \\
\hline $\begin{array}{l}\text { BL_GetCrossRowCompositeY } \\
\text { (ListIndex, CellIndex) }\end{array}$ & BlockList.dll & $\begin{array}{l}\text { Returns a total difference in } \mathrm{Y} \text { - } \\
\text { coordinate of segments in one or } \\
\text { more cells }\end{array}$ \\
\hline $\begin{array}{l}\text { BL_GetCrossColumnCompositeX } \\
\text { (ListIndex, CellIndex) }\end{array}$ & BlockList.dll & $\begin{array}{l}\text { Returns a total difference in X- } \\
\text { coordinate of segments in one or } \\
\text { more cells }\end{array}$ \\
\hline $\begin{array}{l}\text { BL_GetSumCrossRowCompositeY } \\
\text { (ListIndex) }\end{array}$ & BlockList.dll & $\begin{array}{l}\text { Returns the sum of the } \\
\text { BL_GetCrossRowCompositeY's for } \\
\text { all the cells in the list that cross the } \\
\text { Y-coordinate of the cell node }\end{array}$ \\
\hline $\begin{array}{l}\text { BL_GetSumCrossColumnCompositeX } \\
\text { (ListIndex) }\end{array}$ & BlockList.dll & $\begin{array}{l}\text { Returns the sum of the } \\
\text { BL_GetCrossColumnCompositeX's } \\
\text { for all the cells in the list that cross } \\
\text { the X-coordinate of the cell node }\end{array}$ \\
\hline
\end{tabular}


Table 4 (Continued)

\begin{tabular}{|c|c|c|}
\hline Function(arguments) & File & Result \\
\hline $\begin{array}{l}\text { BL_GetCrossRowCompositeLength } \\
\text { (ListIndex, CellIndex) }\end{array}$ & BlockList.dll & $\begin{array}{l}\text { Returns the length of the contour } \\
\text { associated with a cell in the list that } \\
\text { crosses the Y-coordinate of the cell } \\
\text { node }\end{array}$ \\
\hline $\begin{array}{l}\text { BL_GetCrossColumnCompositeLength } \\
\text { (ListIndex, CellIndex) }\end{array}$ & BlockList.dll & $\begin{array}{l}\text { Returns the length of the contour } \\
\text { associated with a cell in the list that } \\
\text { crosses the X-coordinate of the cell } \\
\text { node }\end{array}$ \\
\hline BL_GetRowBoundary(Row) & BlockList.dll & $\begin{array}{l}\text { Returns the position of the Row } \\
\text { boundary indicated by Row }\end{array}$ \\
\hline BL_GetColumnBoundary(Column) & BlockList.dll & $\begin{array}{l}\text { Returns the position of the Column } \\
\text { boundary indicated by Column }\end{array}$ \\
\hline $\begin{array}{l}\text { BL_PointInsideContour (ListIndex, X, } \\
\text { Y) }\end{array}$ & BlockList.dll & $\begin{array}{l}\text { Returns True if }(X, Y) \text { is inside the } \\
\text { contour indicated by ListIndex }\end{array}$ \\
\hline BL_GetRowNodePosition(Row) & BlockList.dll & $\begin{array}{l}\text { Returns the Y position of the node } \\
\text { of the Row indicated by "Row" }\end{array}$ \\
\hline BL_GetColumnNodePosition(Column) & BlockList.dll & $\begin{array}{l}\text { Returns the X position of the node } \\
\text { of the Column indicated by } \\
\text { "Column" }\end{array}$ \\
\hline BL_GetRowBoundaryCount() & BlockList.dll & $\begin{array}{l}\text { Returns the number of row } \\
\text { boundaries in the grid }\end{array}$ \\
\hline BL_GetColumnBoundaryCount() & BlockList.dll & $\begin{array}{l}\text { Returns the number of column } \\
\text { boundaries in the grid }\end{array}$ \\
\hline BL_GetRowNodeCount() & BlockList.dll & $\begin{array}{l}\text { Returns the number of row nodes in } \\
\text { the grid }\end{array}$ \\
\hline BL_GetColumnNodeCount() & BlockList.dll & $\begin{array}{l}\text { Returns the number of column } \\
\text { nodes in the grid }\end{array}$ \\
\hline BL_GetCellArea(Column, Row) & BlockList.dll & $\begin{array}{l}\text { Returns the area of the cell } \\
\text { indicated by Column, Row }\end{array}$ \\
\hline $\begin{array}{l}\text { BL_FractionOfLine(ListIndex, } \\
\text { CellIndex) }\end{array}$ & BlockList.dll & $\begin{array}{l}\text { Returns the fraction of the total } \\
\text { length of the contour inside the cell } \\
\text { indicated by CellIndex }\end{array}$ \\
\hline BL_FreeVertexList() & BlockList.dll & $\begin{array}{l}\text { Attempts to free all memory } \\
\text { associated with a list of verticies } \\
\text { and returns True if the function } \\
\text { succeeds }\end{array}$ \\
\hline BL_FreeAllBlockLists() & BlockList.dll & $\begin{array}{l}\text { Attempts to free all memory } \\
\text { allocated by the BlockList PIE and } \\
\text { returns True if the function } \\
\text { succeeds }\end{array}$ \\
\hline
\end{tabular}


Table 4 (Continued)

\begin{tabular}{lll}
\hline \multicolumn{1}{c}{ Function(arguments) } & \multicolumn{1}{c}{ File } & \multicolumn{1}{c}{ Result } \\
\hline BL_GetErrorCount() & BlockList.dll & Returns the number of errors \\
BL_CheckVersion(First_Digit, & BlockList.dll & $\begin{array}{l}\text { Returns True if the version number } \\
\text { of the BlockList PIE is greater than } \\
\text { Second_Digit, Third_Digit, }\end{array}$ \\
$\begin{array}{l}\text { Fourth_Digit) } \\
\text { or equal to the version number } \\
\text { passed in the arguments }\end{array}$ \\
\hline
\end{tabular}

\section{Installation instructions}

All the PIEs described in this report may be installed by placing the dynamic link libraries (dll's) in the Argus InterwarelArgusPIE directory or a subdirectory of it. In cases where the PIE has a help system, the help system files (with the extensions .hlp and .cnt) must be placed in the same directory as the dll. Normal practice is to create a subdirectory in the ArgusPIE directory with the same name as the dll except without the dll extension and place the files there. For example, the Utility PIE would normally be installed as "Argus Interware/ArgusPIE/Utility/Utility.dll".

Some of the commands or functions described in this report are now implemented in the Utility.dll whereas previously they were implemented in several different dll's. The new versions replace and often improve upon the previous versions so the dll's containing the previous versions must be removed when installing the new versions. The dll's that have been replaced are EditContoursPie.dll, EditDataLayer.dll, GriddedImport.dll, JoinContoursPie.dll, MoreConversions.dll, EvalAtXY.dll, and RotateCells.dll. If the conflicting dll's are not removed, the Utility.dll can not be used.

Two of these PIEs (JoinContoursPie.dll and MoreConversions.dll) were written by the author prior to joining the USGS. Two of them (EditContoursPie.dll, RotateCells.dll) were documented as part of version 3 of the MODFLOW GUI (Winston, 1999). Three more (GriddedImport.dll, EvalAtXY.dll, and EditDataLayer.dll) were documented as part of version 4 of the MODFLOW GUI (Winston, 2000).

\section{Navigating}

A number of the dialog boxes described in this report display spatial data and allow the user to zoom in on or out of an area of interest or to pan to a different location.

Standardized buttons have been used for these operations. To zoom out all the way, click the Zoom Extents button Q. To zoom in by a factor of two, click the Zoom In button 이. To zoom out by a factor of two, click the Zoom Out button $\Theta$ J. To zoom to a specified region, click the Zoom button 9 and select to region to zoom in on. To select the region, click the mouse in one corner of the region, hold down the mouse button while moving to the opposite corner, and release the mouse button. To pan, click the Pan button 에 the mouse button while moving the mouse to a new position. 


\section{Acknowledgments}

I would like to thank Allen M. Shapiro and Bernard J. Stolp for their helpful reviews of this manuscript. I would also like to thank Leonard Konikow, Clifford Voss, Alden Provost, George Z. Hornberger, Allen Shapiro, and Martha Scholl for helpful suggestions.

\section{Interpolation Methods}

Several new interpolation methods are included in Utility.dll. The new methods have two advantages over existing interpolation methods in Argus ONE.

1. They are much faster.

2. They can incorporate anisotropy.

To test the speed of the new interpolation methods, a test case was constructed with a grid containing approximately 500 cells. The grid had one parameter that was linked to a data layer containing approximately 900,000 points (from a digital elevation model). When the interpolation method used for the data layer was one of the new methods, coloring the grid the first time took about 7 seconds. If the Argus ONE window was minimized and then maximized again, recoloring the grid took about 1 second. If the default interpolation method for the data layer was used, coloring the grid took about 1 minute regardless of whether it was being colored for the first time or was being recolored. Other interpolation methods all took even longer.

There are 10 new interpretation methods as listed below.

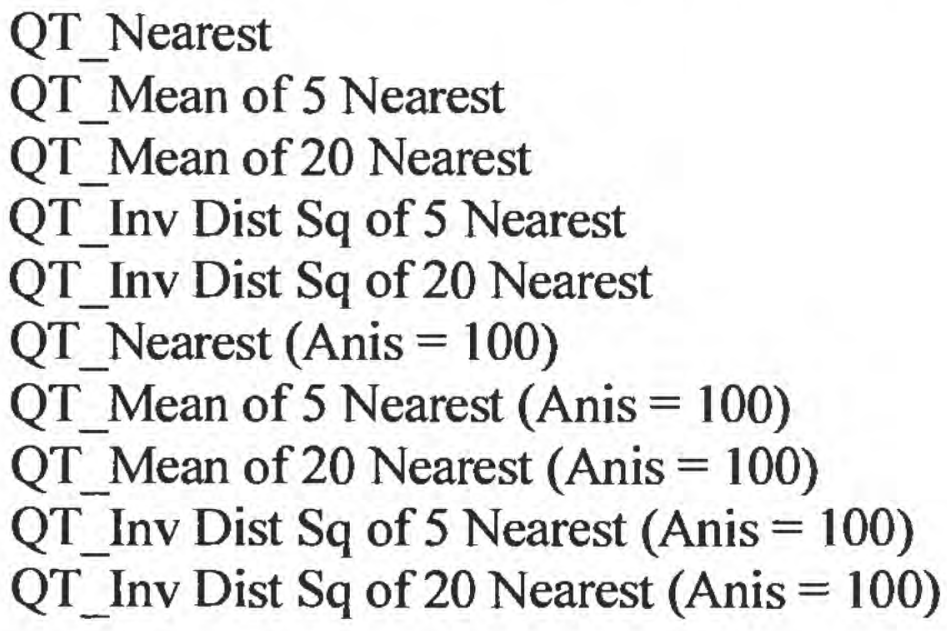

QT_Nearest returns the value of the data point that is closest to the location for which a value is requested. This is exactly equivalent to the NN2D method (except faster). In the event that two locations are equally distant from the location for which a value is requested, an arbitrary choice between the values will be made.

QT_Mean of 5 Nearest finds the five data points that are closest to the location for which a value is requested and returns their mean value. However, it is possible that there will be additional data points that are no further way from the desired location as the point that is the fifth most distant from it. If that is the case, all data points that are at the same distance as the fifth most distant data point will be included in the calculation.

QT_Mean of 20 Nearest is the same as QT_Mean of 5 Nearest except that it uses the 20 closest points rather than the five closest points. 
QT_Inv Dist Sq of 5 Nearest retrieves the five closest data points to the search location in the same way as QT_Mean of 5 Nearest and then calculates a weighted mean of the data points. The weights applied to each data point are the inverses of the square of their distances from the search location. (In the event that one or more data points lies exactly at the search location, the inverse of the distance can not be calculated so the value that is returned is the mean of the values of all the data points that lie exactly at the search location.)

QT_Inv Dist Sq of 20 Nearest is the same as QT_Inv Dist Sq of 5 Nearest except that it uses the 20 closest points rather than the five closest points.

QT_Nearest (Anis = 100) is the same as QT_Nearest except that the Y coordinates of all the data points and the search location are multiplied by 100 before performing any operations. This would be useful for performing interpolations in cross sectional models where aquifer properties are typically much more continuous in the horizontal than in the vertical direction.

The remaining anisotropic methods are the same as their isotropic equivalents except that the Y coordinates are all multiplied by 100 . For instance, QT_Mean of 5 Nearest (An is $=100$ ) is the same as QT_Mean of 5 Nearest except that the Y coordinates of all the data points and the search location are multiplied by 100 before performing any operations.

The reason for the "QT" in the name of all these interpolation methods is that the data are stored in a data structure known as a Quadtree (Stephens, 1998). The advantage of using a Quadtree is that it can make retrieving data of the sort required by interpolation methods much faster than would be required by a sequential search through the data.

\section{Commands}

The commands described here are all implemented in the file Utility.dll. The commands appear in the Argus ONE menus. Commands are in bold type to help distinguish them from the surrounding text although other items may also in bold type. In the description of commands, a vertical bar (|) is used separate menu items from submenu items. For example, and instruction to select PIEs|Edit means to select the PIEs menu item and then to select the Edit submenu item from the PIEs menu.

\section{Edit}

\section{Edit Contours}

The Edit Contours command is used to edit the positions of individual vertices in contours. To use it, select PIEs|Edit|Edit Contours. You will be prompted for a layer name. Select the layer for which you wish to edit a vertex position. All the contours from the layer will be read and displayed. Click on any vertex, and a dialog box will appear in which you can edit the vertex position. When you are done, click $\mathrm{OK}$. The layer will be cleared and a new set of contours will be written with the new vertex positions.

Users of the Edit Contours command should be aware of the following characteristics of the command before using it.

1. The contour parameter values will be the same as they were originally; however, any parameters that were set using Expressions will now be specified in the contour itself. To go back to having the contour value being set by the expression for the parameter, edit the contour and delete the parameter value. 
2. The command does not check for contours that are illegal in Argus ONE. A contour is illegal if it crosses another contour and "Allow Intersection" is not turned on for that layer. A contour is also illegal if it crosses itself even if "Allow Intersection" is turned on. Argus ONE will not accept illegal contours so illegal contours will be lost if you specify contours that are illegal. For this reason, it is best to back-up your file before using the Edit Contours command. If you anticipate wanting to import contours that touch, you should select "Allow Intersection" beforehand.

\section{DeclutterContours}

Reducing the number of vertices in a layer can make the export process faster. If the vertices are much more closely spaced than the grid spacing, reducing the number of vertices may have little effect on the model inputs. To use this command, select PIEs|Edit|Declutter Contours. A dialog box will appear with a list of information layers. Select the Domain Outline or Information layer in which you wish to reduce the number of vertices. Another dialog box will appear in which you should enter the desired vertex spacing and an angle used to control which vertices are removed.

When you click OK the contours on the layer will be processed so that vertices that are closer together than the desired spacing will be eliminated if the "Delete nodes based on spacing" check box is checked. However, if the "Delete Node based on angle" check box is checked, nodes will only be deleted if the angle at the node exceeds the limit you specify. The effect of Declutter Contours is illustrated in Figure 1. Figure 1A shows contours with very closely spaced vertices many of which have been eliminated in Figure 1B by using the Declutter Contours command.
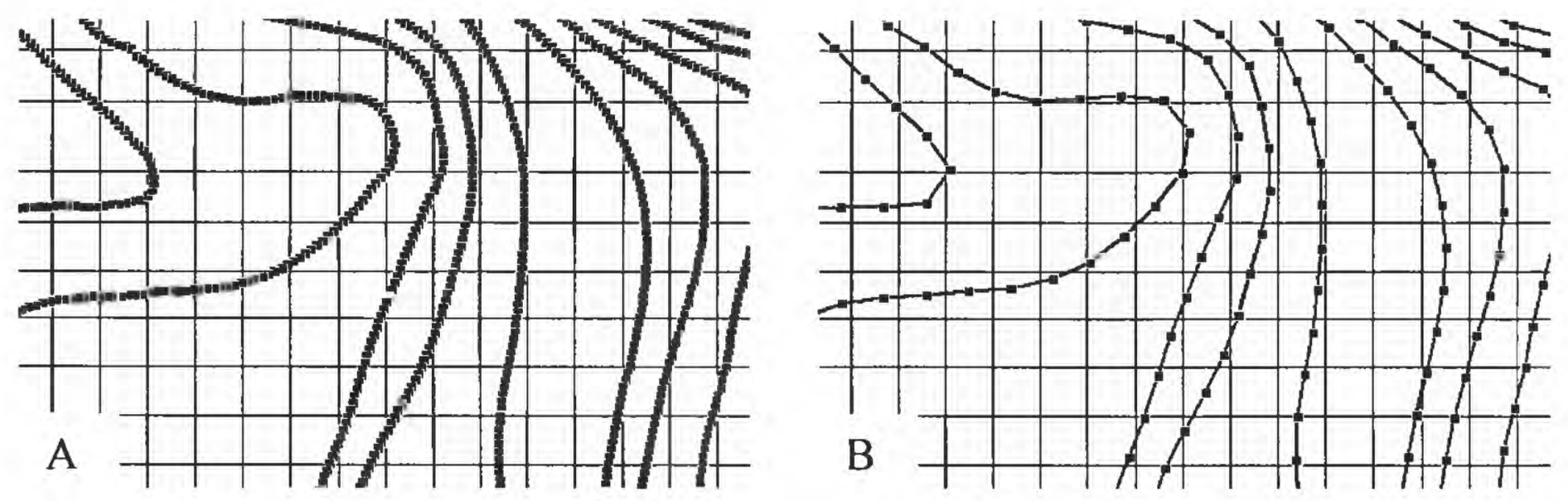

Figure 1. Effect of using the Declutter Contours command. (A) Before. (B) After.

A word of warning is in order about the Declutter Contours command. After removing vertices, some contours that previously did not overlap may overlap and the geometric relationships among contours may differ. For example, in Figure 2, the $-200 \mathrm{ft}$ contour has been reduced to a single point that is no longer inside the $-150 \mathrm{ft}$ contour as it was before. The user should check the contours for such problems after using this command. 

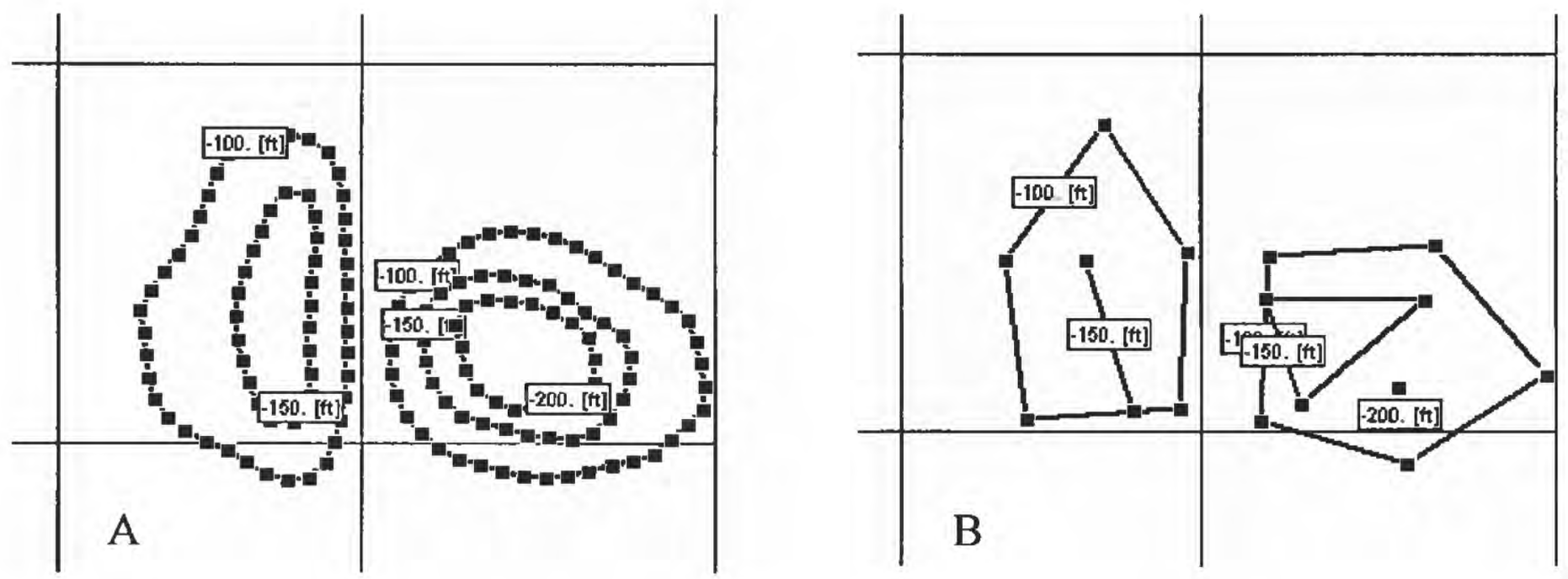

Figure 2. If used improperly, the Declutter Contours command can result in contours that overlap or that no longer show correct geometric relationships. (A) The contours before the Declutter Contours command was used. (B) The contours after the Declutter Contours command was used. Note that the $-200 \mathrm{ft}$. contour is outside the $-150 \mathrm{ft}$ contour after the Declutter Contours command was used.

Both the Join Contours (to be described next) and Declutter Contours commands first clear the layer before writing to it. If there is an error when writing the data to the layer, this will result in loss of data. An error might occur in the Declutter Contours command if some of the contours cross themselves after some vertices are removed. Thus it is a good idea to save the Argus ONE file before running either of these PIEs. It usually would not cause an error if contours crossed each other because the Declutter Contours command automatically turns on "Allow Intersection" for the information layer that it is using. However, "Allow Intersection" is not allowed for Domain Outline layers so for them, it would cause an error.

\section{Join Contours}

You may sometimes wish to join contours together to make it easier to manipulate them. To join contours together, select PIEs|Edit|Join Contours. A dialog box will appear listing the information layers. Select the layer in which you want to combine contours. The contours will be read from the layer. If any two contours have exactly the same starting or ending point and all their parameter values are the same, those contours will be combined into a single contour (Figure 3). 

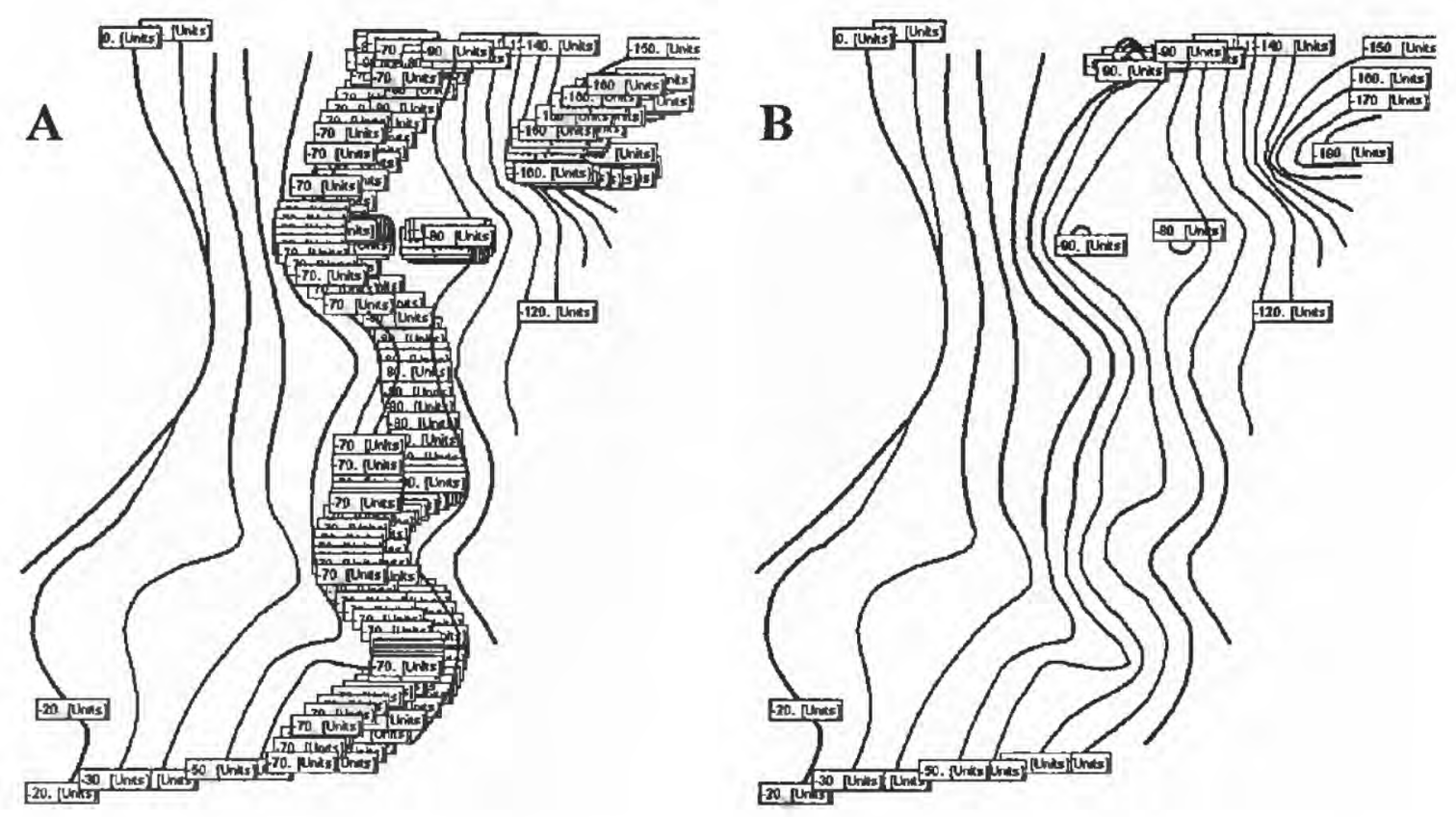

Figure 3. Effect of the Join Contours command (A) Before the Join Contours command is applied, there are a large number of contours that meet at their ends and that have exactly the same contour values. Each contour has its own label. (B) After the Join Contours command is applied, these contours have been joined together.

If two contours have starting or ending points that are even slightly different, the contours will not be combined. To ensure that such contours are combined, make sure the "Special|Allow Intersection" is checked. Then move the end of one of the contours close to the end of the other. The cursor will change to a hollow cross to indicate that it has detected another vertex. When you release the mouse, the vertex will be placed exactly over the position of the other vertex.

\section{Edit Grid}

The Edit Grid command is used for creating and editing grids. The user can move or rotate the grid, or add, delete, or move rows and columns. The user can also subdivide rows and columns or specify the width or positions individual rows and columns. To ensure numerical stability and accuracy during model computation, it is a good idea to make sure that the ratio between the widths of adjacent rows and columns is less than or equal to a 1.5 (Anderson and Woessner, 1992). The Edit Grid command can automatically adjust an existing grid or create a new one that meets this critierion.

To start the Edit Grid command, select PIEs|Edit|Edit Grid. You will be prompted for the name of the grid layer whose grid you want to edit or you can choose to create a new grid layer. You will also be prompted for the type of grid (Block-Centered or GridCentered). If you decide to create a new grid layer, you can use either existing Domain Outline and Density layers or you can create new ones. Once you have selected or created the grid layer, the main dialog box will appear. It will have a copy of the grid (if there is one) on the grid layer you selected. You can edit the grid in this dialog box. 


\section{Moving the Grid}

To move the grid, you change the coordinates of the grid origin: the corner of the grid at column 1 , row 1 . There are two ways to change the coordinates of the grid origin. boxes.

1. Type the values of the $\mathrm{X}$ and $\mathrm{Y}$ coordinates of the grid origin in the appropriate edit

2. Click on the arrow button $\downarrow$. Next, click inside the grid. The cursor will change to a hand pointer. While holding the mouse button down, move to the location where you would like the grid to be. A gray outline of the grid will move along with the mouse. When you release the mouse button, the coordinates of the grid origin will be moved to reflect the position you specified.

Rotate the Grid

There are three ways to rotate the grid.

1. Type the angle of the grid into the edit box labeled Grid Angle.

2. Rotate the knob at the bottom of the dialog box.

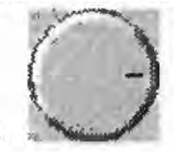

3. Click on the rotate button $\mathrm{G}$. Next, click inside the grid. The cursor will change to a hand pointer. While holding the mouse button down, move to the location where you would like the grid to be. A gray outline of the grid will rotate along with the mouse. When you release the mouse button, the angle of the grid will be changed to reflect the position you specified.

\section{Moving Grid Lines}

To move grid lines, click on the move column 幽 or move row $\frac{ \pm}{\mathrm{v}}$ button. Then move the mouse over the column or row line you wish to move. The cursor will change to an image similar to those on the buttons when the cursor is over a column or row. Click the mouse button and hold it down. As you move the mouse, an image of the column or row will follow the mouse. When you release the mouse, the column or row will be moved. You can also move grid lines by specifying column and row positions or by specifying column and row widths as described later in this section.

\section{Adding Grid Lines}

To add row or column grid lines, click on the add column 1 or add row button. Then move the mouse to where you wish to add a column or row. When you click the mouse button and release it, a new column or row will be added at the mouse position. As you move the mouse, an image of the new column or row boundary will follow the mouse. You can also add grid lines by specifying column and row positions or by specifying column and row widths as described later in this section.

\section{Changing the Width of Rows or Columns}

To change the width of rows or columns, click on the column width $\Theta_{\text {or row }}$ height 1 button. Then move the mouse over one of the columns or rows whose width you wish to change. Hold down the left mouse button and, if desired, move the mouse to another column or row. When you release the mouse button, a dialog box will appear where you can enter a new width for all the selected columns or rows. As you move the mouse with the button down, the selected columns or rows will be shown in gray. You can also change the width of rows or columns by specifying column and row positions or by specifying column and row widths as described later in this section. 


\section{Deleting Grid Lines}

To delete grid lines, click on the delete button $x$. Then move the mouse over one of the grid lines that you wish to delete and click the mouse button. As you move the mouse with the button down, the selected columns or rows will be shown in gray and the cursor will change to an $\mathrm{X}$. You can also delete grid lines by specifying column and row positions or by specifying column and row widths as described later in this section.

\section{Subdividing Rows or Columns}

To subdivide rows or columns, click on the subdivide button $\#$. Then move the mouse over one of the columns or rows whose width you wish to change. Hold down the left mouse button and, if desired, move the mouse to another column or row. When you release the mouse button, a dialog box will appear where you can enter the number of columns and rows that you wish to subdivide the selected columns or rows into. If you enter a number greater than one for either and click the OK button, each selected columns and row will be subdivided into the number of columns and rows you specify. As you move the mouse with the button down, the selected columns or rows will be shown in gray. You can also subdivide rows or columns by specifying column and row positions or by specifying column and row widths as described below.

Specifying Row and Column Positions

To specify row and column positions, click on the Column/Row Positions button 16. In the dialog box, you can increase or decrease the number of columns or rows and enter new positions for columns or rows. The positions don't need to be in order because they will be arranged automatically.

\section{Specifying Row and Column Widths}

To specify row and column widths, click on the Column/Row Widths button $|6|$ In the dialog box, you can increase or decrease the number of columns or rows and enter new widths for the columns or rows. You can drag rows in the table to rearrange them.

\section{Adjusting Row and Column Boundaries}

According to Anderson and Woessner (1992), the maximum ratio between adjacent row or column widths should usually be less than or equal to 1.5 . They also recommend that the maximum ratio between the length and width of individual cells should be less than 10 . Numerical difficulties in solving the model equations arise less frequently in models with grids that meet these criteria.

To adjust the row and column boundaries so that the ratio between the size of adjacent rows and columns is less than a specified value, first, enter a number greater than 1 in the grid smoothing criterion edit box. This number represents the maximum desired ratio between the sizes of adjacent columns or rows. Then click the smooth grid button $\Omega$. The positions of the grid lines will be changed in an attempt to satisfy the criterion. If it doesn't succeed, the maximum ratio in the grid will be displayed in a warning message. Clicking the button again may reduce the ratio further.

\section{Creating Grids}

If there isn't a grid already, you can add row or column grid lines to create it as described above. If you want to create a grid using information from the Domain Outline and Density layers, first set the grid angle and smoothing criterion to the desired value. Then click on the Magic Wand button $\approx$. A dialog box will appear in which you may select the 
names of the Domain Outline and Density layers and the type of grid to be created. When you have done this, the grid will be created.

When you click the OK button, your edited grid will be imported into Argus ONE. However, all the cells in the grid will be active. To deactivate the cells outside the domain outline, click with the Argus ONE "Magic Wand" button inside the domain outline and choose "Deactivate". When the operation has finished, all cells outside the Domain outline will be shaded to show they are inactive.

\section{Edit Data}

To use this procedure, select Files|Edit|Edit Data.

You must have at least one parameter on the data layer before it can be edited. If you attempt to edit a data layer that has no parameters, you will get an error message about the problem.

If there are no data points on the data layer, will get a warning message but you will still be able to edit the data layer. When you edit the data layer, you can: move data points, change the value or values of data points, add data points, and/or delete data points.

When you are finished editing the data points click on the OK button to accept the changes or the Cancel button to discard them. The data points appear both on the Graphical tab and the Table tab.

The OK and Cancel buttons will both close the dialog box but if the $\mathrm{OK}$ button is clicked the changes made in the dialog box will be kept. Otherwise they will be discarded. Other buttons on the Graphical tab are used for navigating.

You can move data points, change their values, add new data points or delete existing data points on both the Graphical and Table tab.

On the Graphical tab, you can do these tasks as follows. To move a data point or edit its values, make sure that the Select point $\boldsymbol{k}$ button is depressed and click on the data point. Then edit the $\mathrm{X}$ and $\mathrm{Y}$ coordinates in the edit boxes or edit the parameter values in the table. To add a data point, first make sure that the Add point \pm button is depressed and then click at the location where you would like to add a data point. Then edit the parameter values and $\mathrm{X}$ and $\mathrm{Y}$ coordinates and data values in the dialog box. To delete a data point, first make sure that the Delete point $x$ button is depressed and then click on the data point you would like to delete.

On the Table tab, you can do these tasks as follows. To move a data point, locate the data point in the table of data points and edit the $\mathrm{X}$ and $\mathrm{Y}$ coordinates in the table. To change the values of a data point, locate the data point in the table of data points and edit the parameter values in the table. To add a data point, increase the number of data points by clicking on the up arrow next to the control that shows the number of data points. Then edit the parameter values and $\mathrm{X}$ and $\mathrm{Y}$ coordinates in the table. To delete a data point, locate the data point you would like to delete and drag it to the bottom of the table. Then decrease the number of data points by clicking on the down arrow next to the control that shows the number of data points.

If you have data in a spreadsheet you can copy them to the clipboard and paste them in the table on the Table tab by clicking on the Paste from Clipboard button. You can also read the data from a file by clicking the Read from file 1 button. Several file formats can be used as described below. 
If the file or clipboard contains data for more parameters than exist on the data layer, extra columns will be created in the table. You can drag the columns to new positions to determine which values will actually be used.

Data to be read from a file or pasted from the clipboard must either be in the format of an Argus ONE contour as described in the Argus ONE documentation (Argus Interware, Inc., 1997, p. 104 to 108) or they must have the following format.

1. Any line in the data whose first character is a "\#" will be considered a comment and will be used to assign names to imported parameters.

2. Each line in the data will begin a new row in the table except for comment lines.

3. The data may be either in a tab-delimited format or a comma/space-delimited format.

4. In tab-delimited format, the data value that will appear in each cell in a row is separated from the data value in the next cell in the row by a tab character.

5. In comma/space-delimited format, the data value that will appear in each cell in a row is separated from the data value in the next cell in the row by one or more tab characters, commas, or spaces. If you wish to import strings that include spaces while still using the comma/space delimited format, enclose the strings in quotation marks.

Note: If you copy data from a commercial spreadsheet to the clipboard, the data will generally be in a tab-delimited format.

\section{Create Parameters in Multiple Layers}

This command allows you to add identical parameters to multiple layers in one step. To activate this command, select PIEs|Edit|Create Parameters in Multiple Layers. A dialog box will appear on which are listed those layers that can have parameters. Make sure that the check box next to each layer is checked for every layer to which you wish to add the parameter. In the bottom half of the dialog box, enter the parameter name, type, units, and value (expression). If you click the OK button, a parameter of the type you defined will be added to each of layers you selected. However, for any data layer, the type of the parameter will always be a real number regardless of the type that you specify because that is the only type allowed in data layers.

\section{Set Multiple Parameters}

This command allows you to set the expressions of multiple parameters in multiple layers in one step. To activate this command, select PIEs|Edit|Set Multiple Parameters. A dialog box will appear with a hierarchical arrangement of layers and their parameters. A parameter will not appear on the list if it has been locked so that its expression can not be modified. Make sure that the check box is checked next to every parameter whose expression you wish to change. Set the value (expression) for the parameters in the edit box and click the $\mathrm{OK}$ button to set the expressions. The expressions are not checked for correctness; the user is responsible for ensuring their validity. In the case of complicated expressions, it may be helpful to create the expression first using the Argus ONE expression editor. Then copy the expression to the clipboard and apply it to multiple parameters using this command. 


\section{Import}

\section{Import Gridded Data}

Import Gridded Data is a command for Argus ONE that facilitates importing gridded data into Argus ONE. This capability is helpful for importing data from existing finite difference models into Argus ONE. A new data layer will be created with data points at the center of the cells in an existing grid in Argus ONE. Each data point will have one or more associated parameters. The PIE provides an easy method for creating these data points and for setting the values of the data points.

You must create a grid in Argus ONE before you can use the Import Gridded Data command because the command will attempt to create data points at the center of each of the cells in the grid. Consult the Argus ONE documentation for how to create a grid. Once you have created a grid, select the PIEs|Import|Import gridded data menu item. The main window is where the gridded data are prepared for importing into Argus ONE. If the checkbox labeled Export each data set to a separate layer is checked, each data set that is imported into Argus ONE will be imported as a separate layer. By default, they are all imported into the same layer.

In the edit box labeled Layer Name is the name of the data layer on which the data should be placed. If there is no layer by this name, it will be created. If it does exist, you will be given a choice of overwriting the existing data or selecting a new layer name that does not exist. This edit box is only enabled if all the data sets will be imported into a single layer.

The Number of data sets is the number of data values associated with each new data point. Each value will be stored in an Argus ONE parameter. You must also assign names to the Argus ONE Parameter names. Parameter names must be unique within the layer. However, if each data set will be imported into a separate layer, enter the layer names in the table rather than parameter names.

There may be some cells for which you do not wish to create data points. If these cells have one or more values that do not occur in the rest of the data, they can be ignored. Set the number of data values and then set the values to be ignored. If the data will all be imported to a single layer, only the first data set is checked in this way so the rest of the data sets must have valid data for all locations that are valid in the first data set.

When pasting data from the clipboard, the values for individual data points must be in one of the following formats:

- Tab-delimited: each row of the table starts on a new line. Each data value on the line is separated from the next by a single tab.

- Comma, space-delimited: each row of the table starts on a new line. Each data value on the line is separated by one or more commas, spaces, or tabs.

In either case, lines beginning with a "\#" character will be considered comments and ignored. If you copy data from a spreadsheet to the clipboard and paste them to the table, it should work with either format. Data that are copied to the clipboard from spreadsheets are generally in tab-delimited format.

In some cases, the data you want to import may have multiple lines of data in the file that all belong on the same row of the model. If the Multiple data lines per grid row check box is checked, each row of the grid will be filled before going on to the next row of the grid. 
After copying the desired data to the clipboard, click on Paste from clipboard button to paste them in the table. Be sure to use the right format for the data as described above.

You may also read data from one or more files each containing a single twodimensional array that you want to import. Click the Read data from files button and select the files. If required, the number of data sets will be increased to accommodate the number of files you select. Each parameter will be assigned the name of the file from which its data were imported. (You can then edit those names if you wish.) The first file to be imported will be placed in the table on the tab that is active. As each file is read, the selected tab will be changed so that each file goes in its own table.

The table contains the data to be placed in the model. Note that the numbers of the rows and columns in the table will match the numbering of the rows and columns in the grid. For example, if in Argus ONE, the Grid direction is Negative X, the columns in Argus will be numbered from right to left instead of left to right. However, the columns in the table will still be numbered from left to right.

Clicking the OK button will create one or more data layers with the data you have entered.

\section{Import Points from Spreadsheet}

Import Points from Spreadsheet allows you to import point contours into Argus ONE from a spreadsheet-like format. To use it, select PIEs|Import|Import Points from Spreadsheet. Then select the information layer into which you wish to import point contours. A dialog box will appear in which you can enter the $\mathrm{X}$ and $\mathrm{Y}$ coordinates of the point contours and all the parameter values. If you have a spreadsheet with this data, you can arrange the data in the spreadsheet in the same order as shown in the table and copy the data to the clipboard. Then paste the data by clicking the Paste from clipboard button. The data may be either in a tab-delimited format or it can be delimited by commas and/or spaces as described in the section entitled "Import Gridded Data". If the data are in a text file, you can also read them from that text file by clicking the Read from file button.

When the contours are about to be imported, the user will be prompted to either retain the existing contours or delete them. This option is provided because two or more point contours can exist at the same location and importing new point contours can conceal the presence of existing contours at the same location.

\section{Import Contours from Spreadsheet}

Import Contours from Spreadsheet allows you to import contours into Argus ONE from a spreadsheet-like format. To use it, select PIEs|Import|Import Contours from Spreadsheet. Then select the information layer into which you wish to import point contours. A dialog box will appear in which you can enter the $\mathrm{X}$ and $\mathrm{Y}$ coordinates of the contours in one table and the parameter values in a second table. If you have a spreadsheet with this data, you can arrange the data in the spreadsheet in the same order as shown in the table and copy the data to the clipboard. Then paste the data by clicking the Paste from clipboard button. The data may be either in a tab-delimited format or they can be delimited by commas and/or spaces as described in the section entitled "Import Gridded Data". If the data are in a text file, you can also read them from that text file by clicking the Read from file button. 
The number of contours to be imported and the maximum number of vertices in any of the contours are set in a pair of edit boxes in the lower left corner of the Contour Coordinates tab of the Import Contours dialog box. If data are pasted into the table, the number of contours and the maximum number of vertices per contour will be automatically updated to match the pasted data. Similarly, on the Parameter Values tab, the number of columns will be adjusted to accommodate the data pasted into the table. However, the Argus ONE layer into which the data are to be imported can only hold data for the parameters that already exist. Therefore, the user should either insure that sufficient parameters already exist in the layer to hold the data to be imported before starting to import it or may rearrange the columns in the table by dragging them to new positions so that the correct data are imported.

\section{Sample DEM Data}

Sample DEM data reads Digital Elevation Models (DEMs) in the format described in the DEM data users guide (U.S. Department of the Interior, U.S. Geological Survey, 1992) (http://rockyweb.cr.usgs.gov/nmpstds/demstds.html). The 1:250K DEMs at http://edcwww.cr.usgs.gov/doc/edchome/ndcdb/ndcdb.html are in this format. The DEMs are displayed as bitmaps in the Universal Transverse Mercator (UTM) projection. To use it, select PIEs|Import|Sample DEM data.

When you select Sample DEM data, you are first prompted for whether you wish to import just the outline of the DEM, or data relative to a grid or mesh. If you wish to import data relative to a grid, you must specify whether the grid is "Block-Centered" or "GridCentered".

When you click the OK button, another dialog box will appear. In it select File|Open to select a DEM file to read. If you choose to import just the outline of the DEM into Argus ONE, you will be prompted whether you wish to read just the outline of the DEM or the full DEM. As the file is read, it will be displayed in the dialog box.

The size of the bitmap showing the DEM is determined by the size of the display area when the DEM is read. If the size of the display area is changed, you can select File|Refresh to create a new bitmap at the current size. Although the user can zoom in and out, this is accomplished by stretching the bitmap rather than by redrawing it so zooming, by itself, does not change the size of the bitmap. To save a bitmap, select File|Save as BMP or File|Save as JPEG. (Argus ONE can read the BMP but not the JPEG files)

If the check box labeled High elevations are red is checked, elevations are color coded from red at the highest elevation to purple at the lowest elevation. If it is not checked, the color scale is reversed.

It is possible to read more than one DEM at a time. To do so, just select multiple DEM files when you select File|Open. However, if the DEMs come from zones that use different central meridians to calculate the UTM coordinates, you will be prompted to choose which central meridian you wish to use.

If you wish to emphasize the coastline, it is a good idea to leave the checkbox labeled Make locations with elevations of 0 white checked. However, for areas that have elevations below sea level, you may wish to uncheck this checkbox.

When determining the central meridian for calculating the UTM coordinates, all UTM zones are treated as being exactly 6 degrees wide. This is not correct for a few zones. The area where this is not true is from 0 to 42 degrees east north of 72 degrees north and from 0 to 12 degrees east between 56 and 64 degrees north. Because the author is unaware 
of any available DEMs in this area in the format accepted by this program, no effort has been made to address this issue.

If you click the OK button, the data are imported into Argus ONE at blocks, nodes, or element centers. You may choose to use either a mean value for the block node or element, the closest DEM data value to the point of interest, the highest value in the area around the point of interest, or the lowest value in the area around the point of interest. For blockcentered grids, such as the MODFLOW grid (Harbaugh, Banta, Hill, and McDonald, 2000), the data are imported at the center of the block. For grid-centered grids, such as the HST3D grid (Kipp, 1997), the data are imported at the grid nodes.

The ability to convert DEMs to colored bitmaps might be of interest to those who do not have Argus ONE. Because this aspect of the PIE does not rely on Argus ONE, a standalone program, DEM2Image.exe, was created (Appendix 6).

\section{Copy Tri Mesh/Copy Quad Mesh}

To use these commands, select PIEs|Import|Copy Tri Mesh or PIEs|Import|Copy Quad Mesh. Then select a pair of Tri Mesh or Quad Mesh layers. The mesh on one of the layers will be copied to the other layer. Only the nodes and elements will be copied. Parameters and parameter values will not be copied.

\section{Convert}

\section{Contours To Data}

To use this procedure, select Files|Convert|Contours To Data. You will then be prompted to select the name of an Information or Domain Outline layer. After you have selected it you will be prompted to select the name of a new or existing data layer.

The contours on the Information or Domain Outline layer will be copied to the data layer. A data point will be placed at the position of each vertex of the contours.

All the data points for a contour will have the same value even if the value of the original contour is set by an expression that varies along the length of the contour.

\section{Data to Contours}

Data to Contours converts data points on a data layer to point contours on an information layer. To use it, select PIEs|Convert|Data to Contours. Then select the data layer whose data points you wish to convert.

When the contours are about to be imported, the user will be prompted to either retain the existing contours or delete them. This option is provided because two or more point contours can exist at the same location and importing new point contours can conceal the presence of existing contours at the same location.

\section{Reverse Contours on Clipboard}

Reverse Contours on Clipboard reverses the order of the vertices in a contour. To use it, cut one or more contours to the clipboard. Then select PIEs|Convert|Reverse Contours on Clipboard. Select the layer in which you wish the contours to be placed and click the OK button. The same warnings that apply to Edit Contours also apply to Reverse Contours on Clipboard. 


\section{Mesh Objects To Contours}

Mesh Objects To Contours allows you to select nodes or edges of elements and import them into Argus ONE as contours on information layers. To use it, select PIEs|Convert|Mesh Objects To Contours. Then select the mesh layer from which you wish to import data followed by an information, map, or domain outline layer into which you wish to import data. A dialog box will appear in which you can select nodes or sides of elements. If the arrow button $\$$ is depressed, you can select nodes or sides by clicking on them with the mouse. If the lasso button $ㄹ$ is depressed, you can select nodes by encircling them with the mouse button depressed. In either case, if the shift button is depressed when you click down with the mouse, the selected nodes or sides will be added to removed from the list of selected nodes or sides rather than replacing them.

When you click on the $\mathrm{OK}$ button, the contours will be created. Contours can not branch so if the selected items allow a contour to go in several directions at a node, an arbitrary decision will be made as to which way the contour should go.

\section{Mesh To Contours}

Mesh to Contours converts each element in a triangular or quadrilateral finiteelement mesh to a closed contour on an information layer. To use it, select PIEs|Convert|Mesh to Contours. These contours should not be used to assign properties to nodes where two or more contours intersect! Attempting to do so may produce unpredictable results because the system must determine arbitrarily which of the contours to use in assigning properties to such nodes.

\section{Hidden Commands}

Some of the commands described in this report have been "hidden". They do not normally appear in the Argus ONE menu structure. This was done because these commands circumvent protections built into Argus ONE to prevent users from inadvertently changing or deleting information that usually should not be changed or deleted.

It is possible to make the hidden commands described in this report visible. To do so, create a text file in the same directory as the Utility.dIl PIE whose command you want to reveal. Name the file Utility.ini. Make the first line of the file "Show". The next time you start Argus ONE, the hidden commands in that PIE will be visible in the menu structure. To hide the functions again, delete the Utility.ini file or change its first line to something other than "Show." "Show" is case insensitive.

\section{Set Parameter Locks}

Set Parameter Locks is hidden because if an option for a parameter is locked, it generally means that users should not make ill-considered changes to it.

Set Parameter Locks can be used to lock or unlock multiple parameters at one time. Once the command has been revealed as described above, it can be activated by selecting PIEs|Set Parameter Locks. A dialog box will appear with a hierarchical arrangement of layers and parameters. The user should select the parameters for which something should be locked or unlocked. The checkboxes at the bottom of the dialog box will be unchecked for all parameter locks which are not set for all the selected parameters. Similarly, the checkboxes will be checked for all parameter locks which are set for all the selected 
parameters. The checkboxes will be gray if some of the selected parameters have the related feature locked and others do not. After selecting the parameters to change, the user should check or uncheck the check boxes to make the desired changes to the parameters. When the user clicks the OK button all the selected parameters will have their locks changed as the user specifies using the checkboxes. If any of the checkboxes is in a gray state, that lock will not be changed for any of the selected parameters.

\section{Delete Multiple Layers}

Delete Multiple Layers is hidden because it allows layers that have been locked to prevent deletion to be deleted easily. Because deleting locked layers without proper consideration can destroy large amounts of data and make a model unusable, this command is somewhat dangerous.

Once the Delete Multiple Layers command has been revealed as described at the beginning of this section, it can be activated by selecting PIEs|Delete Multiple Layers. A dialog box will appear with a series of check boxes with layer names next to them. The user must select the layers to delete by checking the check boxes. If the user then clicks the OK button, the user will be warned that the layers will be deleted and given a chance to change the decision to delete. If the user still chooses to delete the layers, they will be deleted.

\section{Functions}

Functions in Argus ONE can be accessed through the expression editor. The Argus ONE documentation describes how to show the expression editor. When the expression editor is visible, there is a list of function categories followed by a list of layers. Under the list of function categories, one of the categories is PIEs. All of the functions described in this document are found under the PIEs category.

\section{Utility_CheckVersion}

Utility_CheckVersion(First_Digit, Second_Digit, Third_Digit, Fourth_Digit) returns True if the version number of the Utility dynamic link library is greater than or equal to the version number passed in the arguments. To check the version number of the dynamic link library right-click on Utility.dll, select "Properties", and go to the version information tab.

\section{EvalRealAtXY, EvallntegerAtXY, EvalBooleanAtXY, and EvalStringAtXY}

EvalRealAtXY, EvalIntegerAtXY, EvalBooleanAtXY, and EvalStringAtXY allow an expression to be evaluated at a specified $X$, Y location. The four functions return a real number, integer, Boolean, and string respectively. The expression is passed to the function as a quoted string. It is up to the user to make sure that the expression is correctly formatted and that the value returned by the function is compatible with the type of the parameter to which it is assigned. If desired, the user may specify the layer on which the expression should be evaluated. The formats of the commands are as follows:

EvalRealAtXY(X, Y, "Expression_As_Quoted_String", ["Layer_Name_As_Quoted_String"]) 
EvalIntegerAtXY(X, Y, "Expression_As_Quoted_String", ["Layer_Name_As_Quoted_String"])

EvalBooleanAtXY(X, Y, "Expression_As_Quoted_String", ["Layer_Name_As_Quoted_String"])

EvalStringAtXY(X, Y, "Expression_As_Quoted_String", ["Layer_Name_As_Quoted_String"])

\section{Rotated $X$, and Rotated $Y$}

Rotated $\mathbf{X}$, and Rotated $\mathbf{Y}$ are functions that can take row or column positions provided by Argus $\mathrm{ONE}$ along with the grid angle and convert them to $\mathrm{X}, \mathrm{Y})$ coordinates. This could be done entirely by an export template, but using a PIE can make the export template simpler and easier to understand and perhaps faster too.

Rotated $\mathrm{X}$ has three arguments: $\mathrm{X}, \mathrm{Y}$, and GridAngle. $\mathrm{X}$ is a column position. $\mathrm{Y}$ is a row position. GridAngle is the angle of the grid. Rotated $\mathbf{X}$ returns the $\mathrm{X}$-coordinate of the point defined by the intersection of the row and column positions. Rotated $\mathbf{Y}$ is just like Rotated X except that it returns the Y-Coordinate.

The first export template in Appendix 5 uses Rotated $\mathbf{X}$ and Rotated $\mathbf{Y}$ to export the row and column numbers or each cell in a grid followed by the $\mathrm{X}$ and $\mathrm{Y}$ coordinates of the four corners of the cell. The template can be used to reproducing the grid in other programs. Users may modify the template to meet their specific requirements.

The second template in Appendix 5 uses Rotated $\mathbf{X}$ and Rotated $\mathbf{Y}$ to export a contour that surrounds a single cell. The contour can be used as a domain outline in models that define a subgrid in a single cell.

\section{GetMyDirectory}

GetMyDirectory.dll is a PIE that returns the name of the directory in which it is installed. The name of the function will be the name of the dll itself without the extension. Typically, two programs are installed in the same directory as GetMyDirectory.dll: SelectChar.exe, and WaitForMe.exe.

SelectChar.exe is a console program that simulates a keyboard event based on its command line arguments. In the following [Char] is used to represent any character and [N] represents any number. If a command line argument contains "Alt-[Char]", SelectChar.exe will simulate depressing the Alt key and the [Char] key simultaneously. If a command line argument contains "Ctrl-[Char]", SelectChar.exe will simulate depressing the Ctrl key and the [Char] key simultaneously. If a command line argument contains "Chr-[N]", SelectChar.exe will simulate depressing the key whose key code is [N]. For any other command line argument SelectChar.exe will simulate depressing the first character in the command line argument. If SelectChar.exe is called with no command line arguments, it will simulate a carriage return.

WaitForMe.exe is a program that can run until it is closed. It is only possible to run one copy of WaitForMe.exe at a time. If you attempt to run a second copy, the first copy will be activated instead. It can be closed by activating it and typing a carriage return. The purpose of this is to start the program before doing something else and then activate it again when that "something else" is done. You can then close it by running SelectChar.exe. An external program, such as UCODE, can be waiting for WaitForMe.exe to finish executing before continuing. 
These three files make it possible for an external program such as UCODE (Poeter and Hill, 1998) to run MODFLOW or SUTRA through Argus ONE and wait until MODFLOW has finished executing before continuing. In the MODFLOW GUI 3 and Sutra PIE 1.0.3 or later, if you define a Global variable named "Calibrate" and set its value to True $(=1)$. Argus ONE will activate and then close WaitForMe.exe in the directory containing GetMyDirectory.dll after running MODFLOW. It will also allow the DOS window in which MODFLOW or Sutra ran to close. For this to work, GetMyDirectory.dll, SelectChar.exe, and WaitForMe.exe must all be in the same directory and must be in the ArgusPIE directory or a subdirectory of the ArgusPIE directory.

\section{ReadFileValue functions}

The functions in the ReadFileValue PIE have the function names shown below except that they may have a prefix such as "MODFLOW_" or "SUTRA_".

The functions in the ReadFileValue PIE are primarily intended to be used with an external program such as UCODE (Poeter and Hill, 1998) that is running Argus ONE. RF_Clear_Files and RF_Save_Files are called in the export templates for MODFLOW and SUTRA.

\section{RF_Get_Value_From_File}

In RF_Get_Value_From_File(Key, Default_Value, [FileName]), "FileName" is an optional parameter. If "FileName" is not specified, "FileName" will be given the value of "Default.txt". The function checks if it has already opened "FileName". If it hasn't, it reads "FileName" into memory. If "FileName" does not exist, it creates a new version of the "FileName" in memory. It then checks for "Key" in "FileName". If any full line in "FileName" is equal to "Key", the next line is converted to a double-precision real number and returned as the result of RF_Get_Value_From_File. If none of the lines in "FileName" is equal to "Key", "Key" is added to the file followed by "Default Value" and the result of RF_Get_Value_From_File is "Default_Value".

"FileName" can be either a fully qualified path name or just a file name. If it is just a file name, ReadFileValue.dll will look for "FileName" in the current directory. The file that ReadFileValue.dll will check must consist of alternating lines of Keys and numbers. The first line must be a key. Blank lines and comments are not allowed.

RF_Clear_Files

RF_Clear_Files ( clears all files that the ReadFileValue.dll currently has in memory.

\section{RF_Save_Files} disk.

RF_Save_Files() saves all files that the ReadFileValue.dll currently has in memory to

\section{RF_CheckVersion}

RF_CheckVersion(First_Digit, Second_Digit, Third_Digit, Fourth_Digit) returns True if the version number of the PIE is greater than or equal to the version number passed in the arguments. The version number of the PIE may be checked by right clicking on the dll, selecting "Properties" and going to the version information tab. 


\section{Conversion functions}

Kipp (1987) provides conversion formulas for converting from metric units to US customary units. These formulas were programmed into functions listed in Table 3. No explanation of these functions is needed beyond that in the table so they are not listed individually here. These conversions supplement those in the Unit Conversions PIE on the Argus Interware web site (http://www.argusint.com/).

\section{Hidden Functions}

Argus ONE gives PIE developers the ability to "hide" functions. If a function is hidden, it will still work properly but it will not show up in the list of PIE functions. This helps keep inexperienced users from using functions improperly that have a high potential for misuse. The functions in this section can only be used in export templates. For most of them, this is because they either allocate memory that must be released using a different function or use memory allocated by a call to a previous function Others display dialog boxes that would be inappropriate for use with expressions for layer parameters.

To make the hidden functions visible create a text file in the same directory as the PIE whose functions you want to reveal. Name the file the same as the dll except change the extension from dll to ini. Make the first line of the file "Show". The next time you start Argus ONE, the functions in that PIE will be visible in the expression editor. To hide the functions again, delete the "ini" text file or change its first line to something other than "Show." "Show" is case insensitive.

\section{OKCancel Functions}

The OkCancel PIE contains functions that can be used to ask the user questions and get responses in an export template. The OkCancel PIE is used in the export template for SUTRA if the bandwidth of the mesh appears high. In such cases, the user is asked if they want to continue exporting or stop. The second template in Appendix 5 also uses the OkCancel PIE.

\section{IsOK}

IsOK(Message, [HideCancel]) displays "Message" in a dialog box with "Yes", "No", and "Cancel" buttons. If the optional "HideCancel" parameter is set to true, the "Cancel" button, is not shown. It returns True if the user clicks on "Yes". Otherwise it returns False. The following is an example export template using the IsOK function

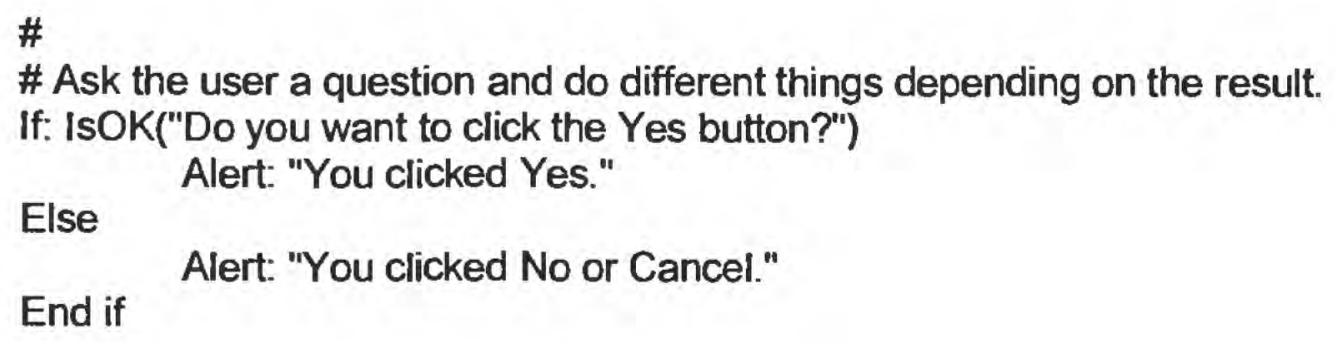

\section{Ok_Add_Radio_Choice}

Ok_Add_Radio_Choice, ok_Get_Radio_Choice, and ok_Radio_Free are functions related to displaying a dialog box with a series of radio buttons and getting a response from 
the user depending on the radio button selected. Ok_Add_Radio_Choice(Message, [Message], [Message], [Message] , [Message]) adds up to five radio buttons to the list of radio buttons that are displayed. The text of each radio button will be "Message".

Ok_Add_Radio_Choice creates, but does not display, the dialog box if the dialog box does not already exist. If the dialog box already exists, ok_Add_Radio_Choice adds Message to the list of choices displayed in the dialog box.

Ok_Add_Radio_Choice returns True if it is successful. It should succeed unless the computer's memory has been exhausted.

\section{Ok_Get_Radio_Choice}

Ok_Get_Radio_Choice(Message, [Choices_Height], [Width], [Question_Height]) displays a dialog box with a question set in the first parameter. There are three optional parameters. The first sets the height in pixels of the box containing the choices. The second sets the width of the dialog box in pixels, and the third sets the height in pixels of the question to be displayed. The choices that the user may select as responses must have previously been set with a call to ok_Add_Radio_Choice. The dialog box is destroyed after the response is obtained in ok_Get_Radio_Choice. The function returns the index of the radio button that the user selected as his or her response. The radio buttons are numbered consecutively starting at zero.

\section{Ok_Radio_Free}

Ok_Radio_Free() destroys the dialog box without displaying it. This function is only needed if ok_Add_Radio_Choice is used without a subsequent ok_Get_Radio_Choice.

The following is an export template using IsOK, ok_Add_Radio_Choice, ok_Get_Radio_Choice, and ok_Radio_Free.

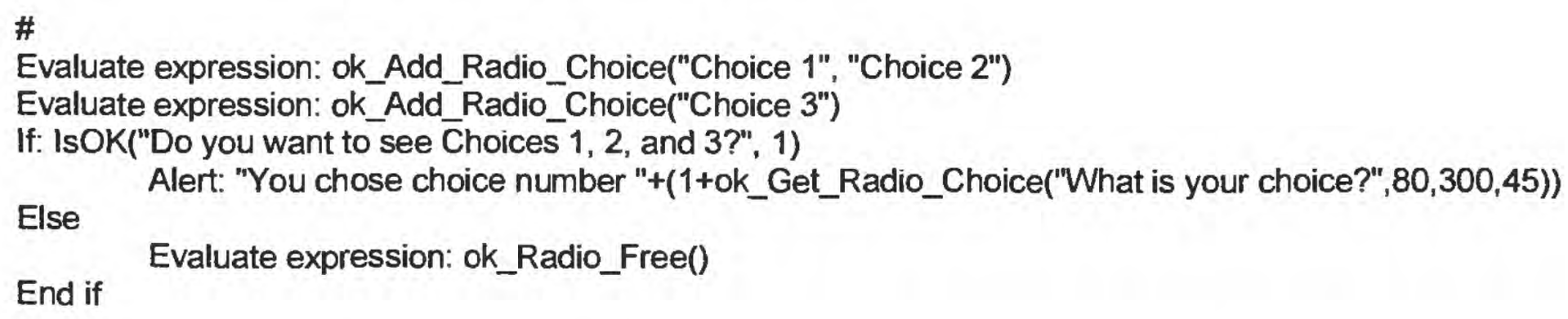

\section{Ok_UserFloat and Ok_UserInteger}

Ok_UserFloat(Message, Response, [Minimum], [Maximum]) and

Ok_UserInteger(Message, Response, [Minimum], [Maximum]) displays a dialog box with a question set by Message and an edit box containing Response. If Minimum and/or Maximum are set, they are the minimum and maximum allowable value of the response. The function returns the value shown in the edit box as a real number or integer when the user closes the dialog box depending on which function was called.

\section{Ok_CheckVersion}

Ok_CheckVersion(First_Digit, Second_Digit, Third_Digit, Fourth_Digit) returns True if the version number of the dynamic link library is greater than or equal to the version number passed in the arguments. The version number of the OkCancel dynamic link library 
may be checked by right clicking on OkCancel.dll, selecting "Properties" and going to the version information tab.

\section{ProgressBar Functions}

The functions in the ProgressBar PIE have the function names shown below except that they may have a prefix such as "MODFLOW_" or "SUTRA_".

The ProgressBar PIE allows you to display a progress bar during the export of a model that advances as the export progresses. It also has a label and a memo box with which messages to the user can be displayed without halting the export process. The contents of the memo can be saved to a text file. The progress bar shows the elapsed time and gives an estimate of the time remaining to complete the export process.

The ProgressBar PIE is used extensively in the export template for MODFLOW-96 (Winston, 2000).

Appendix 2 has an example export template that uses the ProgressBar PIE.

\section{ProgressBarlnitialize}

ProgressBarInitialize(Number, [Show_Cancel]) creates and shows the progress bar and sets the maximum position of the progress bar to Number. Initially the progress bar position is at 0 . If Show_Cancel is true, an "Abort" button will be visible. This function returns True if it succeeds. Any of the remaining ProgressBar functions will fail if ProgressBarInitialize is not called first.

\section{ProgressBarFree}

ProgressBarFree() frees the progress bar and frees all memory associated with the progress bar. This function returns True if it succeeds. If ProgressBarFree(), is not called at the end of the export process, the progress bar will remain visible after the export process is complete.

\section{ProgressBarMax}

ProgressBarMax(Number) sets the maximum value of the progress bar. This is also set in ProgressBarInitialize(Number). This function returns True if the Abort button has not been pressed and the function succeeds.

\section{ProgressBarAdvance}

ProgressBarAdvance() increases the position of the progress bar by one. This function returns True if the Abort button has not been pressed and the function succeeds.

\section{ProgressBarSetMessage}

ProgressBarSetMessage(Message) sets the message in the label displayed in the progress bar to Message. This function returns True if the Abort button has not been pressed and the function succeeds.

\section{ProgressBarAddLine}

ProgressBarAddLine(Message) adds Message to the list of messages in the memo. This function returns True if the Abort button has not been pressed and the function succeeds. 


\section{ProgressBarSaveToFile}

ProgressBarSaveToFile(File_Name) saves the messages in the memo to a text file named File_Name and returns the number of lines in the file it saves.

\section{ProgressBarCheckVersion}

ProgressBarCheckVersion(First__Digit, Second_Digit, Third_Digit, Fourth_Digit) returns True if the version number of the dynamic link library is greater than or equal to the version number passed in the arguments. To check the version number of the ProgressBar dynamic link library right-click on Progressbar.dll, select "Properties", and go to the version information tab.

\section{JoinFiles Functions}

The JoinFiles PIE contains functions for performing operations on files.

The functions in the JoinFiles PIE have the function names shown below except that they may have a prefix such as "MODFLOW_" or "SUTRA_". The JoinFiles PIE is used extensively in the export template for MODFLOW-96 because it allows the template to be more efficient (Winston, 2000). Certain items at the beginnings of the input files are counts of items that occur later in the file. The JoinFiles PIE makes it possible to count the items as they are exported, write the count to a separate file, and then join the files to create a properly formatted, MODFLOW, input file.

\section{Join_Files}

Join_Files(First_File, Second_File, Result_File) appends Second_File to First_File and stores the result in Result_File.

\section{Delete_File}

Delete_File(File_Name) deletes the file indicated by File_Name.

\section{Rename_File}

Rename_File(Old_File_Name, New_File_Name) changes the name of a file from Old_File_Name to New_File_Name.

\section{Split_File}

Split_File('Input_File, First_File, Search_String, Second_File [, Search_String, Third_File] [, Search_String, Fourth_File]...[, Search_String, Thirtieth_File]) Splits a file into up to $\overline{3} 0$ sections. The input file is read one line at a time and saved to $\overline{\text { First_File. If }}$

Search_String is found in a line, First_File is closed and subsequent lines are saved in Second_File and the last line of First_File will be Search_String. If there are additional parameters (Search_String, Third_File, etc.) the remainder of 'Input_File will continue to be searched by occurrences of the new Search_String and split into separate files as before.

\section{Int2Str}

Int2Str(Number) converts a number to its character representation in Base $36 .(0,1, \ldots$ $9, \mathrm{~A}, \mathrm{~B}, \ldots \mathrm{Z}, 10,11, \ldots 19,1 \mathrm{~A}, \ldots .$.$) . This can be useful if a file extension must be less than 3$ characters long and the file extension must have a number. For example, MODFLOW 
(Harbaugh, Banta, Hill, and McDonald, 2000) can be compiled in such a way that only file names less than or equal to eight characters in length with extensions less than or equal to three characters in length will be recognized. If the IBOUND array for each of 100 layers is stored in separate files with names of the form MyModel.il to MyModel.i100, there will be a problem. The last file, MyModel.i100 won't be recognized because the extension has more than 3 characters. In an export template that used Int2Str, the file name could be MyModel.i2s which would be acceptable.

\section{JF_CopyLines}

JF_CopyLines(Old_File_Name, New_File_Name, Line_Count, [Is_Local_File]) creates a new text file the current directory named New_File Name and opens an existing file named Old_File_Name. It then reads Line_Count lines from Old_File_Name and writes them to New_File_Name. If it can do all this, it returns True. Otherwise it returns False. If the optional Is_Local_File parameter is present and is set to False, Old_File_Name represents the full path of the existing file. Otherwise it represents the name of a file in the current directory.

\section{JF_CheckVersion}

JF_CheckVersion(First_Digit, Second_Digit, Third_Digit, Fourth_Digit) returns True if the version number of the PIE is greater than or equal to the version number passed in the arguments. To check the version number of the PIE right-click on JoinFiles.dll, select "Properties", and go to the version information tab.

\section{List functions}

The functions in the List PIE have the function names shown below except that they may have a prefix such as "MODFLOW_" or "SUTRA_.". The List PIE maintains variablesize, zero-based lists (arrays) of real numbers. The number of dimensions in the lists can be either one or three.

It is possible to call the functions in the List PIE with incorrect arguments. In such cases, the List PIE will increment an internal variable that contains a count of the number of errors that have occurred and then return a result. Where possible, the result will indicate that an error occurred but it is not always possible for the function to do this. Users can call the L_GetErrorCount function.get determine the number of errors that have occurred and then take appropriate action.

The List PIE is used extensively in the export template for MODFLOW 96 (Winston, 2000). It isn't needed for MODFLOW-2000.

An example export template using the List PIE is in Appendix 3.

\section{L_Initialize}

L_Initialize() (obsolete) was formerly used to initialize the List PIE. It is no longer needed but is still present for backwards compatibility.

\section{L_CreateNewList}

L_CreateNewList() create a new list and returns the "ListIndex" of the new list. ListIndex is used to refer to a particular list when it is used. If the function is unsuccessful, it increments the error count and returns -1. L_CreateNewList should only be unsuccessful if 
the computer's memory has been exhausted. The values of ListIndex returned by L_CreateNewList are consecutive and start at 0 .

\section{L_SetListSize}

L_SetListSize(ListIndex, Size) adds additional items with values of 0 to the list indicated by ListIndex until the number of items in the list is equal to Size. You can still add additional items to a list after using SetListSize. The function returns True if it is successful, and False if unsuccessful. If it is unsuccessful, it will increment the error count. If ListIndex indicates a list that does not exist, SetListSize will be unsuccessful. If the list is already larger than Size, L_SetListSize does nothing.

\section{L_GetListSize}

L_GetListSize(ListIndex) returns the number of items in the list indicated by ListIndex. The function returns -1 if it is unsuccessful. If ListIndex indicates a list that does not exist, L_GetListSize will be unsuccessful and will increment the error count.

\section{L_FreeAList}

L_FreeAList(ListIndex) deletes all items in the list indicated by ListIndex. It does not delete the list itself so the ListIndicies of lists created after the list that has been emptied do not change. The function returns True if successful, and False if unsuccessful. If ListIndex indicates a list that does not exist, L_FreeAList will be unsuccessful and will increment the error count.

\section{L_AddToList}

L_AddToList(ListIndex, Value) adds an item to the end of the list indicated by ListIndex. The numeric value of the item is Value. Value is stored as a double-precision real number. The function returns the "Index" of the item if successful, and -1 if unsuccessful. "Index" is the position of the item in the list. If ListIndex indicates a list that does not exist or if memory has been exhausted, L_AddToList will be unsuccessful and will increment the error count. The values of "Index" are consecutive and start at zero.

\section{L_GetFromList}

L_GetFromList(ListIndex, Index) returns the item at the position indicated by Index from the list indicated by ListIndex. The function returns 0 if unsuccessful. If ListIndex and Index indicate a list or an item that does not exist, L_GetFromList will be unsuccessful and will increment the error count.

\section{L_SetListltem}

L_SetListItem(ListIndex, Index, Value) sets the value of the item at the position indicated by Index in the list indicated by ListIndex to Value. The function returns True is successful and False if unsuccessful. If ListIndex and Index indicate a list or an item that does not exist, L_SetListItem will be unsuccessful and will increment the error count. For example, if L_GetListSize(ListIndex) returns 5, L_SetListItem(ListIndex, 5, Value) would be unsuccessful but L_SetListItem(ListIndex, 4, Value) would succeed. 


\section{L_DeleteListltem}

L_DeleteListItem(ListIndex, [Index]) deletes the item at the position indicated by Index from the list indicated by ListIndex. This reduces the number of items in the list by one. The function returns True is successful and False if unsuccessful. If ListIndex and Index indicate a list or an item that does not exist, L_DeleteListItem will be unsuccessful and will increment the error count. "Index" is optional. If Index is not included, the last item in the list indicated by ListIndex will be deleted.

\section{L_SortList}

L_SortList(ListIndex) sorts the items in the list indicated by ListIndex from lowest at the beginning of the List to highest at the end of the list. The function returns True if it succeeds and False if it fails. If the list indicated by ListIndex does not exist, L_SortList will fail and will increment the error count.

\section{L_EliminateDuplicates}

If two adjacent items in a list are identical, L_EliminateDuplicates(ListIndex) will delete one of them from the list. Usually, you should call L_SortList or otherwise ensure that the list is sorted before calling L_EliminateDuplicates to eliminate all duplicate entries in the list. The function returns True if it succeeds and False if it fails. If the list indicated by ListIndex does not exist, L_EliminateDuplicates will fail and will increment the error count.

\section{L_IndexOf}

L_IndexOf(ListIndex, Value) returns the position within a sorted list indicated by ListIndex of the first occurrence of Value. Normally, you should call L_SortList or otherwise ensure that the list is sorted before calling L_IndexOf.

If Value is not in the list, it will return the index of the first occurrence of the largest item smaller than Value.

For example if the list contains $0,1,1,3$ : L_IndexOf(ListIndex, 0 ) returns 0 , L_IndexOf(ListIndex, 1) returns 1, L_IndexOf(ListIndex, 2) returns 1, and L_IndexOf(ListIndex, 3) returns 3.

If the list indicated by ListIndex does not exist, $L \_$IndexOf will fail. It will then return -1 and will increment the error count.

\section{L_UnsortedlndexOf}

L_UnsortedIndexOf(ListIndex, Value) returns the position of Value in the list indicated by ListIndex. If it is not found, the function returns -1 . If the list indicated by ListIndex does not exist, L_UnsortedIndexOf will fail. It will then return -1 and will increment the error count.

\section{L_CreateNew3DList}

L_CreateNew3DList(Maximum_X, Maximum_Y, Maximum_Z) creates a new 3D List with dimensions [0..Maximum_X-1, 0..Maximum_Y-1, 0..Maximum_Z-1]. If it succeeds, L_CreateNew3DList returns the ListIndex of the new 3D List. The function returns -1 if unsuccessful. L_CreateNew3DList will be unsuccessful if memory has been exhausted. If the function is unsuccessful, it will increment the error count by 1 . The values of ListIndex returned by L_CreateNew3DList are consecutive and start at 0 . The numbering 
of 3D lists is independent of the numbering of lists created with $\mathrm{L}_{-}$CreateNewList. Thus, the initial calls to L_CreateNew3DList and L_CreateNewList would both return 0 . Initially all items in a $3 \mathrm{D}$ list have a value of 0.

\section{L_FreeA3DList}

L_FreeA3DList(ListIndex) deletes all items from the 3D List indicated by ListIndex and sets Maximum_X, Maximum_Y and Maximum_Z to 0 . The function returns True if successful and False if unsuccessful. If ListIndex indicates a 3D list that does not exist, $\mathrm{L}$ _FreeA3DList will be unsuccessful. If the function is unsuccessful, it will increment the error count.

\section{L_GetFrom3Dlist and L_GetFromOneBased3DList}

L_GetFrom3DList(ListIndex, X_Index, Y_Index, Z_Index) returns the value of the item within the $3 \mathrm{D}$ list indicated by ListIndex at the position indicated by X_Index, Y_Index, and $Z$ Index. The function returns 0 if it fails. If ListIndex, $X$ Index, $Y$ Index, $Z$ Index indicate a $3 \mathrm{D}$ list or an item in a 3D list that does not exist, $\mathrm{L}_{-}$GetFrom $3 \overline{\mathrm{D}} \mathrm{List}$ will be unsuccessful and will increment the error count.

L_GetFromOneBased3DList is identical to L_GetFrom3DList except that the 3D list is treated as having the following limits [1..Maximum_X, 1..Maximum_Y, 1..Maximum_Z] instead of [0..Maximum_X-1,0..Maximum_Y-1, 0..Maximum_Z-1].

\section{L_Set3Dlistltem and L_SetOneBased3DListltem}

L_Set3DListItem(ListIndex, X_Index, Y_Index, Z_Index, Value) sets the value of the item within the 3D list indicated by ListIndex at the position indicated by X_Index, Y_Index,

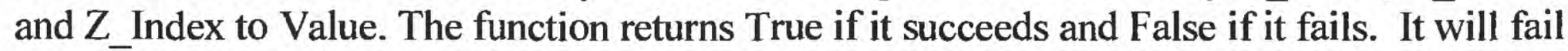
if the $\overline{3} \mathrm{D}$ list indicated by ListIndex does not exist or if the position indicate by $\mathrm{X}$ Index, Y Index, and Z_Index is outside the extents of the 3D List. If it fails, it will increment the error count.

L_SetOneBased3DListItem is identical to L_Set3DListItem except that the 3D list is treated as having the following limits [1..Maximum_X, 1..Maximum_Y,1..Maximum_Z] instead of [0..Maximum_X-1,0..Maximum_Y-1, 0..Maximum_Z-1].

\section{L_ResetA3DList}

L_ResetA3DList(ListIndex) sets all items in the 3D list indicated by ListIndex to 0 . The function returns True if it succeeds and False if it fails. It will fail if the 3D list indicated by ListIndex does not exist. If it fails, it will increment the error count.

\section{L_Add3DLists}

L_Add3DLists(FirstListIndex, SecondListIndex, ResultListIndex) adds each member of the $3 \mathrm{D}$ list indicated by FirstListIndex to the corresponding member of the 3D list indicated by SecondListIndex and places the result in the corresponding member of the 3D list indicated by ResultListIndex. The function will fail and return False if FirstListIndex, SecondListIndex, or ResultListIndex indicate a 3D list that does not exist. It will also fail if all of the 3D lists do not have the same limits. If it fails, it will increment the error count. 


\section{L_Subtract3DLists}

L_Subtract3DLists(FirstListIndex, SecondListIndex, ResultListIndex) subtracts each member of the 3D list indicated by SecondListIndex to the corresponding member of the 3D list indicated by FirstListIndex and places the result in the corresponding member of the 3D list indicated by ResultListIndex. The function will fail and return False if FirstListIndex, SecondListlndex, or ResultListIndex indicate a 3D list that does not exist. It will also fail if all of the 3D lists do not have the same limits. If it fails, it will increment the error count.

\section{L_Multiply3DLists}

L Multiply3DLists(FirstListIndex, SecondListIndex, ResultListIndex) multiplies each member of the 3D list indicated by FirstListIndex by the corresponding member of the 3D list indicated by SecondListIndex and places the result in the corresponding member of the 3D list indicated by ResultListIndex. The function will fail and return False if FirstListIndex, SecondListIndex, or ResultListIndex indicate a 3D list that does not exist. It will also fail if all of the 3D lists do not have the same limits. If it fails, it will increment the error count.

\section{L_Divide3DLists}

L_Divide3DLists(FirstListIndex, SecondListIndex, ResultListIndex) divides each member of the 3D list indicated by FirstListIndex by the corresponding member of the 3D list indicated by SecondListlndex and places the result in the corresponding member of the 3D list indicated by ResultListIndex. The function will fail and return False if FirstListIndex, SecondListlndex, or ResultListlndex indicate a 3D list that does not exist. It will also fail if all of the 3D lists do not have the same limits. If it fails, it will increment the error count.

\section{L_Multipy3DByConstant}

L_Multipy3DByConstant(Listlndex, ResultListIndex, Value) multiplies each member of the $3 \mathrm{D}$ list indicated by ListIndex by Value and places the result in the corresponding member of the 3D list indicated by ResultListIndex. The function will fail and return False if ListIndex or ResultListIndex indicate a 3D list that does not exist. It will also fail if the 3D lists indicated by ListIndex and ResultListIndex do not have the same limits. If it fails, it will increment the error count.

\section{L_Invert3DListMembers}

L_Invert3DListMembers(ListIndex, ResultListIndex) takes the reciprocal of each member of the 3D list indicated by ListIndex and places the result in the corresponding member of the 3D list indicated by ResultListIndex. The function will fail and return False if ListIndex or ResultListIndex indicate a 3D list that does not exist. It will also fail if the 3D lists indicated by ListIndex and ResultListIndex do not have the same limits. If it fails, it will increment the error count.

\section{L_IsSingPrecUniform}

L_IsSingPrecUniform(ListIndex) returns True if all members of the list indicated by ListIndex have the same value after being converted to single precision. The function will fail and return False if ListIndex indicates a 3D list that does not exist. If it fails, it will increment the error count. 
This function is used in the export template for MOC3D to check that the cells in the export subgrid are of uniform size.

\section{L_GetErrorCount}

L_GetErrorCount() returns the number of errors that have occurred in the List PIE since the PIE was loaded or the last call to L_FreeAllLists. Normally an error count greater than 0 indicates that the export process did not go as planned. This function is used in the export template for MODFLOW to check for programming errors in the export template.

\section{FreeAllLists}

L_FreeAllLists() Deletes all items in all lists and all 3D Lists, deletes the lists and sets the error count to 0 . The function returns True if it is successful and False if unsuccessful. L_FreeAllLists should never be unsuccessful. L_FreeAllLists() should be called at the end of any export template that uses the List PIE because it will release memory allocated by the List PIE. If it is not called, the memory will be released when Argus ONE is shut down.

\section{L_CheckVersion}

L_CheckVersion(First_Digit, Second_Digit, Third_Digit, Fourth_Digit) returns True if the version number of the dynamic link library is greater than or equal to the version number passed in the arguments. The version number of the dynamic link library may be checked by right clicking on List.dll, selecting "Properties" and going to the version information tab.

\section{BlockList functions}

The functions in the BlockList PIE have the function names shown below except that they may have a prefix such as "MODFLOW_" or "SUTRA_". The BlockList PIE contains GIS functions deal with the relationships between contours and grid cells. The functions indicate (1) which cells are intersected by a contour, (2) which cells are inside a contour, (3) the order in which cells are intersected by a contour (4) the length of the section of the contour intersected by a cell, (5) the cells next to the ones intersected by the contour but on the other side of the contour, and (6) many other data relating the contours and cells. The BlockList PIE is, by far, the most complicated PIE discussed in this paper. It can provide information that would be difficult to calculate with just an export template. In the MODFLOW GUI (Winston, 2000) the BlockList PIE is used extensively in preparing the input for the Horizontal Flow Barrier (Hsieh, and Freckleton, 1993) and Stream (Prudic, 1989) packages for MODFLOW-96. For MODFLOW-2000, the blocklist functions are called directly by the MODFLOW GUI rather than through an export template and the functions are used for all boundary conditions rather than just the Horizontal-Flow Barrier and Stream packages.

In these functions, ListIndex is used to designate a list of cells intersected by a contour. CellIndex indicates a particular cell in the list. VertexIndex indicates a vertex in a cell. SegmentIndex indicates a segment of a contour within a cell. In any of the functions, invalid values of Listindex, CellIndex, VertexIndex, or SegmentIndex are used, an error will occur.

It is possible to call the functions in the BlockList PIE with incorrect arguments. In such cases, the BlockList PIE will increment an internal variable that contains a count of the 
number of errors that have occurred and then return a result. Where possible, the result will indicate that an error occurred but it is not always possible for the function to do this. Users can call the BL_GetErrorCount function get determine the number of errors that have occurred and take appropriate action.

Appendix 4 contains an example export template that uses the BlockList PIE.

\section{BL_InitializeGridInformation}

BL_InitializeGridInformation(Grid_Layer_Name_as_String, [GridType]) reads the positions of row and columns from the grid layer named Grid_Layer_Name_as_String. Because Grid_Layer_Name_as_String is a string, it must either be surrounded by quotes or be turned into a string by some other method. GridType is an optional parameter that determines how the grid will be treated in subsequent processing. GridType $=0$ indicates a block-centered grid. GridType $=1$ indicates a node-centered grid. The default is a blockcentered grid. GridType need not match the actual type of the grid. For example a blockcentered grid could be used with a GridType of 1 in order to evaluate all information as if it were a node-centered grid in which all the nodal points were at the intersections of the row and column boundaries in the block-centered grid.

\section{BL_AddVertexLayer}

BL_AddVertexLayer(Information_Layer_Name_as_String) reads the contour information from the information layer named Information_Layer_Name_as_String and determines which cells intersect the contours on the information layer. Because Information_Layer_Name_as_String is a string, it must either be surrounded by quotes or be turned into a string by some other method. BL_AddVertexLayer does not erase the information stored in previous calls to $\mathrm{BL}$ Add $\bar{d}$ VertexLayer but instead adds the information in Information_Layer_Name as_String to the information that was read previously. To erase the contour information, call $\mathrm{BL}_{-}$ReInitializeVertexList().

\section{BL_RelnitializeVertexList}

BL_ReInitializeVertexList() deletes all contour information that has already been entered.

\section{BL_GetCountOfCellLists}

BL_GetCountOfCellLists() returns the number of lists of cells. This is normally the sum of all the objects on all the layers that have been added using BL_AddVertexLayer since the last call to BL_ReInitializeVertexList. Each list of cells contains the cells intersected by the contour in the order in which they were intersected along the length of the contour.

\section{BL_GetCountOfACellList}

BL_GetCountOfACellList(ListIndex) returns the number of cells in a list of cells intersected by a contour. The same cell may be intersected by a contour more than once so the cells in this list may not all be unique.

\section{BL_GetCellRow and BL_GetCellColumn}

BL_GetCellRow(ListIndex, CellIndex) and BL_GetCellColumn(ListIndex, Cellindex) return a row or column number in a list of cells. The first cell is the one at the 
beginning of the contour and the cells are listed in order from the beginning to end of the contour.

\section{BL_GetVertexCount}

BL_GetVertexCount(ListIndex, CellIndex) returns the number of vertices associated with a cell. Vertices will be present wherever a contour has a vertex or a contour intersects the cell boundary.

\section{BL_GetVertexXPos and BL_GetVertexYPos}

BL_GetVertexXPos(ListIndex, CellIndex, VertexIndex) and BL_GetVertexYPos(ListIndex, CellIndex, VertexIndex) return the $\mathrm{X}$ and $\mathrm{Y}$ coordinates of a vertex.

\section{BL_SegmentCount}

BL_SegmentCount(ListIndex, CellIndex) returns the number of line segments within a cell. A segment is the section of a contour within a cell that connects two adjacent vertices.

\section{BL_SegmentFirstX and BL_SegmentFirstY}

BL_SegmentFirstX(ListIndex, CellIndex, SegmentIndex) and

BL_SegmentFirstY(ListIndex, CellIndex, SegmentIndex) returns the X and Y coordinates of the first vertex defining a segment.

\section{BL_SegmentSecondX and BL_SegmentSecondY}

BL_SegmentSecondX(ListIndex, CellIndex, SegmentIndex) and BL_SegmentSecondY(ListIndex, CellIndex, SegmentIndex) return the X and Y coordinates of the second vertex defining a segment.

\section{BL_SegmentLength $X$ and BL_SegmentLength $Y$}

BL_SegmentLengthX(ListIndex, CellIndex, SegmentIndex) and BL_SegmentLengthY(ListIndex, CellIndex, SegmentIndex) return the X or Y coordinate of the second vertex defining a segment minus the $\mathrm{X}$ or $\mathrm{Y}$ coordinate of the first vertex defining a segment.

\section{BL_SegmentLength} segment.

BL_SegmentLength(ListIndex, CellIndex, SegmentIndex) returns the length of a

\section{BL_SumSegments $X$ and BL_SumSegments $Y$}

BL_SumSegmentsX(ListIndex, CellIndex) and BL_SumSegmentsY(ListIndex, Cellindex) return the sum of all the differences between the $\mathrm{X}$ or $\mathrm{Y}$ coordinate of the second vertex defining each segment minus the $\mathrm{X}$ or $\mathrm{Y}$ coordinate of the first vertex defining each segment.

\section{BL_SumSegmentLengths}

BL_SumSegmentLengths(Listlndex, CellIndex) returns the sum of the lengths of all the segments in a cell. 


\section{BL_GetCountOfCombinedCellList}

BL_GetCountOfCombinedCellList() returns the number of all the cells intersected by any contour. Each cell in this list has a unique row and column number. The cells are listed in no particular order but no two cells are identical.

\section{BL_GetCellRowFromCombinedList and BL_GetCellColumnFromCombinedList}

BL_GetCellRowFromCombinedList(CellIndex) and

BL_GetCellColumnFromCombinedList(CellIndex) return a row or column in the list of all cells intersected by contours.

\section{BL_GetCountOfCrossRowLists and BL_GetCountOfCrossColumnLists}

BL_GetCountOfCrossRowLists() returns the number of lists of cells in which a contour crosses the $\mathrm{Y}$-coordinate of the cell node. For block-centered grids, the nodal position is at the center of the grid but in grid-centered grids, the nodal position may be offcenter. For the purposes of this function, a contour is considered not to have crossed the center if its first and last vertices in segments within the cell lie on the same side of the Ycoordinate of the cell node. BL GetCountOfCrossColumnLists() similar to BL_GetCountOfCrossRowLists() except that it deals with cells in which a contour crosses the $\mathrm{X}$-coordinate of a cell node.

\section{BL_GetCountOfACrossRowList and BL_GetCountOfACrossColumnList}

BL_GetCountOfACrossRowList(ListIndex) returns the number of cells in which a contour crosses the $\mathrm{Y}$-coordinate of the cell node.

BL_GetCountOfACrossColumnList(ListIndex) is similar to BL_GetCountOfACrossRowList except that it deals with cells in which a contour crosses the X-coordinate of a cell node.

\section{BL_GetCrossRowRow and BL_GetCrossColumnRow}

BL_GetCrossRowRow(ListIndex, CellIndex) returns the row number of a cell in which the contour crosses the Y-coordinate of the cell node.

BL_GetCrossColumnRow(ListIndex, CellIndex) is similar to BL_GetCrossRowRow except that it deals with cells in which a contour crosses the X-coordinate of a cell node. CellIndex must be greater than or equal to zero and less than or equal to the result of BL_GetCountOfACrossRowList or BL_GetCountOfACrossColumnList

\section{BL_GetCrossRowColumn and BL_GetCrossColumnColumn}

BL GetCrossRowColumn(ListIndex, CellIndex) returns the column number of a cell in which the contour crosses the Y-coordinate of the cell node.

BL_GetCrossColumnColumn(ListIndex, CellIndex) is similar to BL_GetCrossRowColumn except that it deals with cells in which a contour crosses the X-coordinate of a cell node. CellIndex must be greater than or equal to zero and less than or equal to the result of BL_GetCountOfACrossRowList or BL_GetCountOfACrossColumnList

\section{BL_GetCrossRowNeighborColumn and BL_GetCrossColumnNeighborRow}

BL_GetCrossRowNeighborColumn(ListIndex, CellIndex) returns the column number of the neighboring cell to the one in which the contour crosses the $\mathrm{Y}$-coordinate of the cell node (Figure 4). BL_GetCrossColumnNeighborRow(ListIndex, CellIndex) is similar to 
BL_GetCrossRowNeighborColumn except that it deals with cells in which a contour crosses the $\bar{X}$-coordinate of a cell node and it returns a row number rather than a column number. CellIndex must be greater than or equal to zero and less than or equal to the result of BL_GetCountOfACrossRowList or BL_GetCountOfACrossColumnList

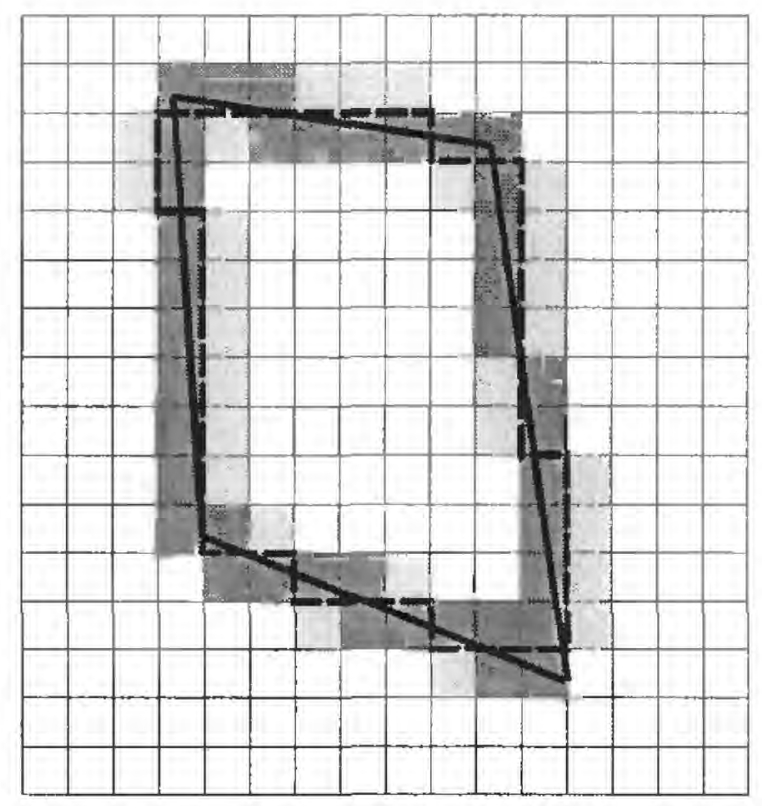

Figure 4. The cells intercepted by the contour (heavy black line) are shown in dark gray. Those cells which are their neighbors, as determined by BL_GetCrossRowNeighborColumn and BL_GetCrossColumnNeighborRow, are shown in light gray except for those which are also intercepted by the contour. The heavy dashed line separates each cell intercepted by the contour from its neighbor or neighbors.

\section{BL_GetCrossRowCompositeY and BL_GetCrossColumnCompositeX}

BL_GetCrossRowCompositeY(ListIndex, CellIndex) returns a total difference in Ycoordinate of segments in one or more cells. The cells over which the sum is returned must be in the same row as the cell that has a contour that crosses the Y-coordinate of the cell node. If there are two such cells in the same row with no other such cells between them on different rows, a maximum or minimum point of the contour between the two cells will be the limit of segments that will be summed for the purpose of this function (Figure 5).

BL_GetCrossColumnCompositeX(ListIndex, CellIndex) is similar to

BL_GetCrossRowCompositeY except that it deals with cells in which a contour crosses the $\mathrm{X}$-coordinate of a cell node and it returns a difference in the X-coordinate. CellIndex must be greater than or equal to zero and less than or equal to the result of

BL_GetCountOfACrossRowList or BL_GetCountOfACrossColumnList 


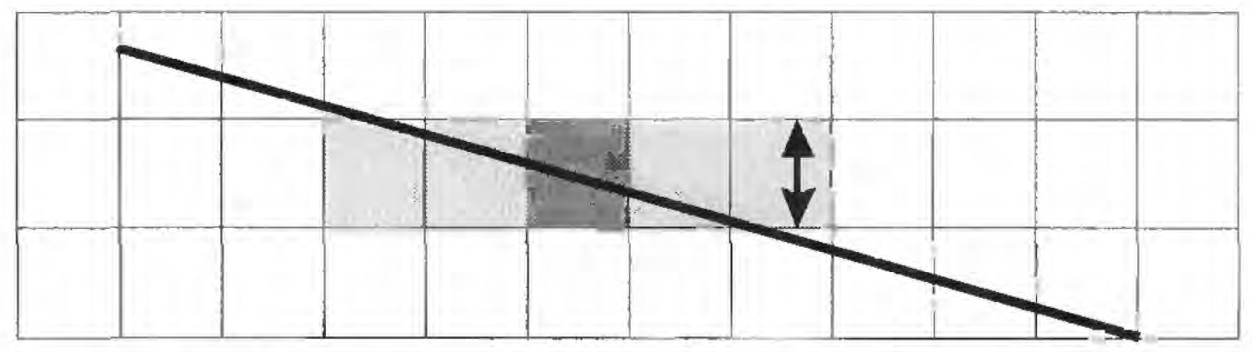

Figure 5. For the cell indicated by the dark gray square, the length returned by BL_GetCrossRowCompositeY is indicated by the double-headed arrow. To determine this, the function retrieved data not only from the dark gray cell but also from the light gray cells.

\section{BL_GetSumCrossRowCompositeY and BL_GetSumCrossColumnCompositeX}

BL_GetSumCrossRowCompositeY(ListIndex) returns the sum of the

BL_GetCrossRowCompositeY's for all the cells in the list that cross the Y-coordinate of the cell node. BL_GetSumCrossColumnCompositeX(ListIndex) is similar to

BL_GetSumCrossRowCompositeY except that it deals with cells in which a contour crosses

the X-coordinate of a cell node. CellIndex must be greater than or equal to zero and less than or equal to the result of BL_GetCountOfACrossRowList or

BL_GetCountOfACrossColumnList

BL_GetCrossRowCompositeLength and

BL_GetCrossColumnCompositeLength

BL_GetCrossRowCompositeLength(ListIndex, CellIndex) is similar to

BL_GetCrossRowCompositeY except that it returns the total length rather than the total

difference in Y-coordinates. BL_GetCrossColumnCompositeLength(Listlndex, CellIndex) is similar to BL_GetCrossRowCompositeLength except that it deals with cells in which a contour crosses the X-coordinate of a cell node. CellIndex must be greater than or equal to zero and less than or equal to the result of BL_GetCountOfACrossRowList or BL_GetCountOfACrossColumnList

\section{BL_GetRowBoundary and BL_GetColumnBoundary}

BL_GetRowBoundary(Row) and BL_GetColumnBoundary(Column) return the position of the Row or Column boundary indicated by Row or Column. These are equivalent to the Row() and Column() functions of Argus ONE.

\section{BL_PointInsideContour}

BL_PointInsideContour(ListIndex, $\mathrm{X}, \mathrm{Y}$ ) returns True if $(\mathrm{X}, \mathrm{Y})$ is inside the contour indicated by ListIndex but is not inside any contours inside the one indicated by ListIndex. (This function may not work if "Allow Intersection" is on and contours cross each other.)

\section{BL_GetRowNodePosition and BL_GetColumnNodePosition}

BL_GetRowNodePosition(Row) and BL_GetColumnNodePosition(Column) return the Y position of the node of the Row indicated by "Row" and the X position of the node of the Column indicated by "Column". These are equivalent to the Argus ONE functions NthRowPos(Row) and NthColumnPos(Column). 


\section{BL_GetRowBoundaryCount and BL_GetColumnBoundaryCount}

BL_GetRowBoundaryCount() and BL_GetColumnBoundaryCount() return the number of row and column boundaries in the grid.

\section{BL_GetRowNodeCount and BL_GetColumnNodeCount}

BL_GetRowNodeCount() and BL_GetColumnNodeCount() return the number of row and column nodes in the grid.

\section{BL_GetCellArea}

Row.

BL_GetCellArea(Column, Row) indicates the area of the cell indicated by Column,

\section{BL_FractionOfLine}

BL_FractionOfLine(ListIndex, CellIndex) gives the fraction of the total length of the line inside the cell indicated by CellIndex (Figure 6).

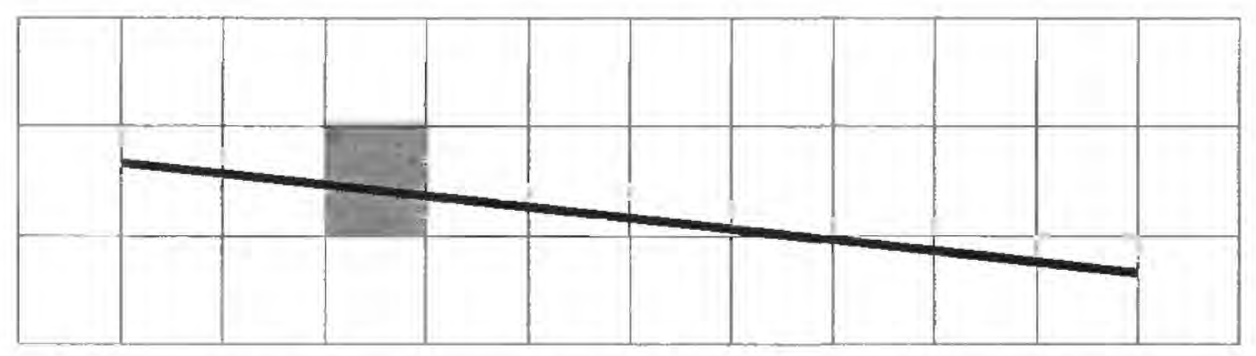

Figure 6. The result of BL_FractionOfLine for the cell indicated by the dark square would be 0.10 because 10 percent of the contour lies within the cell.

\section{BL_FreeVertexList}

BL_FreeVertexList() Frees all memory associated with a list of vertices.

\section{BL_FreeAllBlockLists}

BL_FreeAllBlockLists() Frees all memory associated with the BlockList PIE.

\section{BL_GetErrorCount}

BL_GetErrorCount() returns the number of errors that have occurred since the last time the PIE was initialized in BL_InitializeGridInformation. This function is used in the export template for MODFLOW- $\overline{96}$ as a check for programming errors in the Blocklist PIE.

\section{BL_CheckVersion}

BL_CheckVersion(First_Digit, Second_Digit, Third_Digit, Fourth_Digit) returns True if the version number of the dynamic link library is greater than or equal to the version number passed in the arguments. The version number of the dynamic link library may be checked by right clicking on BlockList.dll, selecting "Properties" and going to the version information tab. 


\section{Conclusions}

One motivation for creating the PIE commands, functions, and interpolation methods described in this report was to provide a mechanism for performing actions that could not be done easily using Argus ONE. For example, when editing a grid in Argus ONE, it was time consuming to create a grid that did not have a uniform cell size and that also did not have abrupt changes in cell sizes. The Edit Grid command makes creating such grids easy. Another motivation was to speed up procedures that could already be done in Argus ONE. A good example of this is the QT_Nearest interpolation method. This gives exactly the same results as the NN2D interpolation method, but it is faster for large data sets because it uses a more efficient algorithm. This report documents the PIEs so that users will have a reference document to which they can turn when attempting to use them in their own work.

\section{References}

Anderson, M.P., and Woessner, W.W., 1992, Applied groundwater modeling, simulation of flow and advective transport: San Diego, California, Academic Press, 381 p.

Argus Interware, Inc., 1997, User's guide Argus ONE ${ }^{\mathrm{TM}}$, Argus Open Numerical Environments - A GIS modeling system, version 4.0: Jericho, NY, Argus Holdings, Limited, $506 \mathrm{p}$.

Harbaugh, A.W., Banta, E.R., Hill, M.C., and McDonald, M.G., 2000, MODFLOW-2000, the U.S. Geological Survey modular ground-water model - User's guide to modularization concepts and the ground-water flow process: U.S. Geological Survey Open-File Report 00-92, 191 p.

Hsieh, P.A., and Freckleton, J.R., 1993, Documentation of a computer program to simulate horizontal-flow barriers using the U.S. Geological Survey modular three-dimensional finite-difference ground-water flow model: U.S. Geological Survey Open-File Report 92-477, $32 \mathrm{p}$.

Kipp, K.L., Jr., 1987, HST3D: A computer code for simulation of heat and solute transport in three-dimensional ground-water flow systems: U.S. Geological Survey, WaterResources Investigations Report 86-4095, 597 p.

1997, Guide to the revised heat and solute transport simulator, HST3D - version 2:

U.S. Geological Survey Water-Resources Investigations Report 97-4157, 149 p.

Konopka, Ray, 1997, Developing custom Delphi 3 components: Albany, New York, Coriolis Group Books, 725 p.

Poeter, E.P., and Hill, M.C., 1998, Documentation of UCODE, a computer code for universal inverse modeling: U.S. Geological Survey Water-Resources Investigations Report 98$4080,116 \mathrm{p}$.

Prudic, D.E., 1989, Documentation of a computer program to simulate stream-aquifer relations using a modular, finite-difference, ground-water flow model: U.S. Geological Survey Open-File Report 88-729, 113 p.

Stephens, Rod, 1998, Ready-to-run Delphi 3.0 algorithms: Wiley, New York, 398 p.

U.S. Department of the Interior, U.S. Geological Survey, 1992, Standards for digital elevation models: Reston, Va., 102 p.

Voss, C.I., Boldt, David, and Shapiro, A.M., 1997, A graphical-user interface for the U.S. Geological Survey SUTRA code using Argus ONE: U.S. Geological Survey, OpenFile Report 97-421, 106 p. 
Winston, R.B., 1999, Upgrade to MODFLOW-GUI: Addition of MODPATH, ZONEBDGT, and additional MODFLOW packages to the U.S. Geological Survey MODFLOW-96 graphical-user interface: U.S. Geological Survey Open-File Report 99-184, 63 p. 2000, Graphical user interface for MODFLOW: Version 4: U.S. Geological Survey Open-File Report 00-315, 27 p. 


\section{Appendix 1: Custom Components Used in PIEs}

Several custom components were developed and used as part of the PIEs described here. These components can also be used in any program or dll created using Borland Delphi. The names of the components follow the naming conventions discussed by Konopka (1997) by including the author's initials or a company name in the name of the component. Detailed descriptions of the properties, methods, and events in the components are contained in Windows help files that come with the components.

\section{TRbwZoomBox}

TRbwZoomBox provides methods for converting real-number coordinates to screen coordinates so that points at those coordinates may be easily displayed.

By default, the positive directions for the real-number coordinate system are to the right (X-axis) and upward (Y-axis). However, the direction of either axis may be reversed. By default, the vertical exaggeration is one but the vertical exaggeration may be set to any positive real number. However, if the vertical exaggeration is set to too high or too low a value, EInvalidOp may be raised when calculating the screen coordinates.

TRbwZoomBox has two embedded components, a TPaintBox and a TShape; the TPaintBox provides the drawing surface. The TShape is only visible during zooming operations. Some of the more important methods of TRbwZoomBox are listed below: displayed:

Zooming methods; methods for changing the magnification at which the data are
AbortZoom
BeginZoom
ContinueZoom
FinishZoom
ZoomBy
ZoomByAt
ZoomOut
SetZoom

Panning methods; methods for changing what area is visible:

\section{BeginPan \\ EndPan}

Coordinate Conversion methods; methods for converting from real-number to screen coordinates and back:

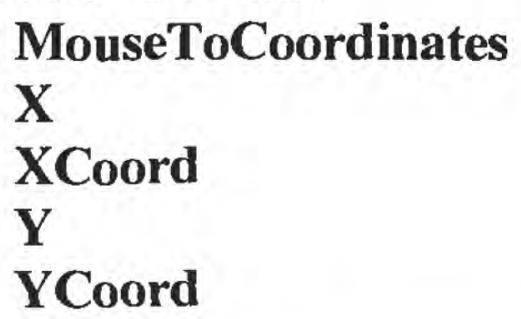

TRbwZoomPoint is a helper-class that store the real-number coordinates. Many of the zooming operations require that TRbwZoomPoint be used to store the coordinates. 


\section{TRbwDynamicCursor}

TRbwDynamicCursor provides a convenient way to draw a cursor at runtime. Assign the cursor hot spot with the HotPointX and HotPointY properties. Draw the cursor on the bitmaps provided in the OnDrawCursor event. Call the RedrawCursor method whenever the cursor needs to be changed.

\section{TArgusDataEntry}

TArgusDataEntry provides data-checking for user-entered data. All data entered in a TArgusDataEntry are first checked to be sure it can be converted to another type of data as specified by the DataType property. In the event that the data can not be converted, characters will be stripped from the end of Text until the conversion is possible. If the DataType is dtInteger or dtReal, it is also possible to check that the numeric representation of Text does not lie outside the range Min..Max. Use CheckMin and CheckMax to turn on or off range checking. OnExceedingBounds and OnExceededBounds can be used to provide custom handling for cases where the data do lie outside the range specified by Min..Max when range checking is on.

TArgusDataEntry was originally developed while the author worked for Argus Interware. It was subsequently adopted by the U.S. Geological Survey.

\section{TRbwQuadTree}

TRbwQuadTree provides methods for storing data associated with $\mathrm{X}$, $\mathrm{Y}$ coordinates in a way that allows the data close to a specific point can be retrieved quickly. Data are added using the AddPoint procedure and removed with the RemovePoint procedure. All the data in the TRbwQuadTree can be removed using the Clear procedure. Data can be retrieved using the FindClosestPointsData, FindNearestPoints, FindPointsInBlock, FindPointsInCircle, and NearestPointsFirstData procedures. Before adding points to the TRbwQuadTree it is best to set Xmax, Xmin, Y $\max$, and Y min to the limits of the data to be added or something close to the limits. Performance may suffer if the limits are greatly different from the actual limits of the data. MaxPoints specifies the maximum number of data points in any one leaf of a TRbwQuadTree. Performance may be affected by MaxPoints in a way that depends on the nature of the task to be performed.

\section{TRbwOctTree}

TRbwOctTree is the three-dimensional equivalent of TRbwQuadTree.

\section{Installation}

To install TRbwZoomBox, TRbwDynamicCursor, TArgusDataEntry, TRbwQuadTree, and TRbwOctTree in Delphi 5, start Delphi 5 and select Component|Install Component. Select the file RbwZoomBox.pas, RbwDynamicCursor.pas, ArgusDataEntry.pas, QuadtreeClass, or OctTreeClass as the "Unit file name" and click the OK button. To install context-sensitive help for the components in Delphi 5 select Help|Customize. On the Contents tab select Edit|Add Files and select TRBWZoomBox.cnt, TDynamicCursor.cnt. or TArgusDataEntry.cnt. Next, on the Index tab select Edit|Add Files and select TRbwZoomBox.hlp, TDynamicCursor.hlp, or TArgusDataEntry.hlp. Then on the Link tab select Edit|Add Files and select 
TRbwZoomBox.hlp, TDynamicCursor.hlp, or TArgusDataEntry.hlp. Finally, select File|Save Project and close the dialog box. 


\section{Appendix 2: Example Export Template for ProgressBar PIE}

\#

Define Variable: OK [Boolean]

Set Variable: OK:= ProgressBarCheckVersion $(1,3,0,0)$

\#

\# Check if the PIE is installed.

If: IsNAN(OK)

Set Variable: OK: $=0$

Alert: "Aborting: You either have an outdated version of the Progress Bar PIE or else you don't have the Progress Bar PIE installed."

Else

\# Check if the PIE is up-to-date.

if: $\mathrm{OK}=0$

End if

Alert: "Aborting: You have an outdated version of the Progress Bar PIE."

End if

If: OK

\# Initialize the progress bar. Set the maximum for the

\# progress bar to the number of blocks.

Evaluate expression: ProgressBarinitialize(NumBlocks(), 1)

\#

\# Set the message shown in the progress bar

Set Variable: OK:= ProgressBarSetMessage("Exporting Blocks")

\#

\# Check if the user has pressed the Cancel button.

If: OK

Redirect output to: \$BaseName\$

Loop for: Blocks

\#

\# Advance the progress bar by one. At the end of the

\# loop over blocks this function will have been called

\# NumBlocks() times.

Set Variable: $\mathrm{OK}:=$ ProgressBarAdvance()

\#

\# Check if the user has pressed the Cancel button.

If: OK

\# Make a test such as a test for an error condition.

If: Column()=Row()

\#

bar memo.

\# If the test suceeds, add a message to the progress

+ Column() + " = Row: " + Row())

Evaluate expression: ProgressBarAddLine("Column: "

\footnotetext{
End if

End file

End loop

End if

\#

\# Save the messages in the progress bar memo to a file.

End if

Evaluate expression: ProgressBarSaveToFile("AFile")

\#

\# Get rid of the progress bar.

End if

Evaluate expression: ProgressBarFree()
} 


\section{Appendix 3: Example Export Template for List PIE}

\#

\# These will be assigned values of Listlndex.

Define Variable: FirstList [Integer]

Define Variable: SecondList [Integer]

\# These will be used to determine how many items are in the list.

Define Variable: Looplndex [Integer]

Redirect output to: \$BaseName\$

Start a new line

\# FirstList is assigned a value of 0 .

Set Variable: FirstList: $=$ L_CreateNewList()

Export expression: FirstList; [G0]

\# SecondList is assigned a value of 1 .

Set Variable: SecondList: = L_CreateNewList()

Export expression: SecondList; [G0]

\# We now add variables to the first and second lists.

Evaluate expression: L_AddToList(FirstList, 5)

Evaluate expression: L_AddToList(FirstList, 7)

Evaluate expression: L_AddToList(SecondList, 9)

\# The number of items in FirstList is 2 and

\# the number of items in SecondList is 1.

Export expression: L_GetListSize(FirstList); [G0]

End line

Export expression: L_GetListSize(SecondList); [G0]

\# This exports all values in the first list.

Start a new line

End line

Export expression: "FirstList" [GO]

Start a new line

Loop for: Variable Looplndex from: 0 to: L_GetListSize(FirstList)-1 step: 1 End loop

Export expression: L_GetFromList(FirstList, LoopIndex); [G0]

End line

\# This exports all values in the second list.

Start a new line

End line

Export expression: "SecondList" [GO]

Start a new line

Loop for: Variable Looplndex from: 0 to: L_GetListSize(SecondList)-1 step: 1

End line End loop

Export expression: L_GetFromList(SecondList, Looplndex); [G0]

\# This does nothing because there are already more than one item if FirstList.

Evaluate expression: SetListSize(FirstList, 1)

\# This adds two more items with a values of 0 to the end of SecondList.

Evaluate expression: L_SetListSize(SecondList, 3)

\# This exports all values in the first list.

Start a new line

End line

Export expression: "FirstList" [G0]

Start a new line

Loop for: Variable LoopIndex from: 0 to: L_GetListSize(FirstList)-1 step: 1

End line End loop

Export expression: L_GetFromList(FirstList, Loopindex); [G0]

\# This exports all values in the second list.

Start a new line

End line

Export expression: "SecondList" [G0]

Start a new line 
End line

Loop for: Variable LoopIndex from: 0 to: L_GetListSize(SecondList)-1 step: 1 End loop

Export expression: L_GetFromList(SecondList, Looplndex); [G0]

\# This deletes all items in FirstList but does not change SecondList.

Evaluate expression: L_FreeAList(FirstList)

\# This exports all values in the first list.

Start a new line

Export expression: "FirstList is Empty"; [GO]

\# This returns 0

End line

Export expression: L_GetListSize(FirstList) [G0]

Start a new line

\# These will cause an error because all loops are executed at least once.

Loop for: Variable Looplndex from: 0 to: L_GetListSize(FirstList)-1 step: 1

\# The error occurs here because FirstList contains nothing.

End loop

Export expression: L_GetFromList(FirstList, Looplndex); [G0]

End line

\# This exports all values in the second list.

Start a new line

End line

Export expression: "SecondList" [GO]

Start a new line

Loop for: Variable LoopIndex from: 0 to: L_GetListSize(SecondList)-1 step: 1

End line End loop

Export expression: L_GetFromList(SecondList, Looplndex); [G0]

\# This sets the value of the second item in SecondList to 20 .

Evaluate expression: L_SetListltem(SecondList, 1, 20)

\# This exports all values in the second list.

Start a new line

Export expression: "SecondList" [G0]

End line

Start a new line

Loop for: Variable Looplndex from: 0 to: L_GetListSize(SecondList)-1 step: 1 End loop

Export expression: L_GetFromList(SecondList, Looplndex); [G0]

End line

\# This deletes the first item of SecondList.

Evaluate expression: L_DeleteListitem(SecondList, 0)

\# This exports all values in the second list.

Start a new line

End line

Export expression: "SecondList" [G0]

Start a new line

Loop for: Variable Looplndex from: 0 to: L_GetListSize(SecondList)-1 step: 1

End line

End loop

Export expression: L_GetFromList(SecondList, Looplndex); [G0]

\# This deletes the last item of SecondList.

Evaluate expression: L_DeleteListltem(SecondList)

\# This exports all values in the second list.

Start a new line

End line

Export expression: "SecondList" [GO]

Start a new line

Loop for: Variable LoopIndex from: 0 to: L_GetListSize(SecondList)-1 step: 1 End loop

Export expression: L_GetFromList(SecondList, Looplndex); [G0]

End line

\# Test if error has occurred. This export template was designed to have one error in it. 


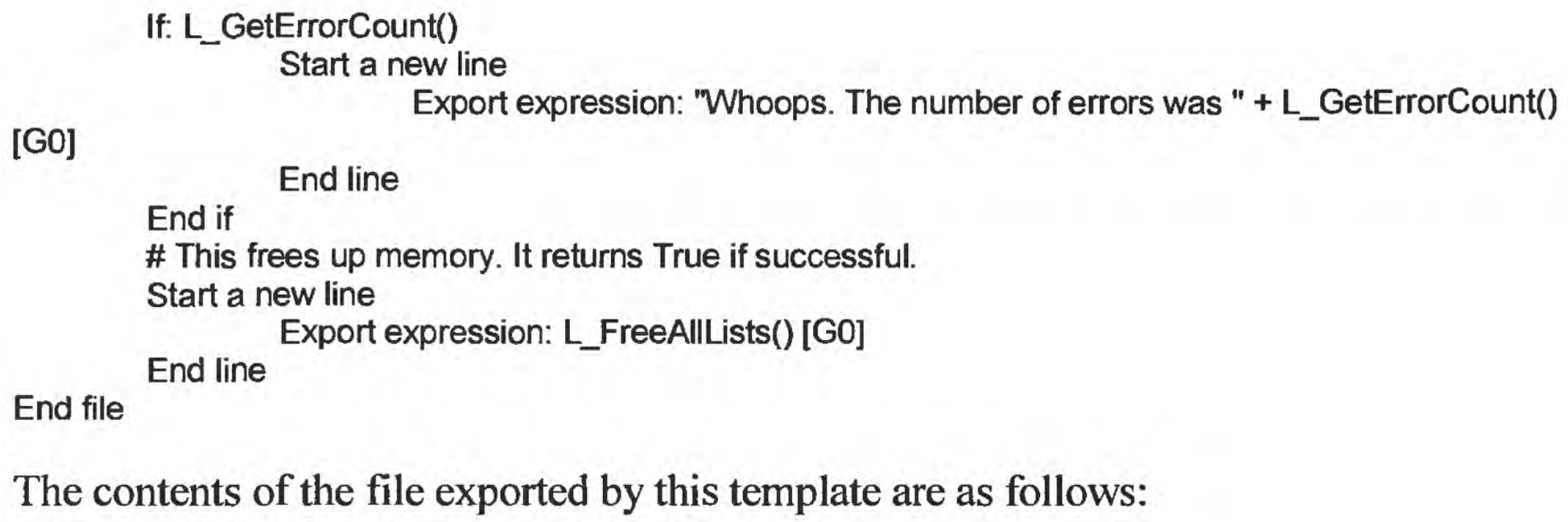

The contents of the file exported by this template are as follows:

0121

FirstList

5. 7 .

SecondList

9.

FirstList

5. 7.

SecondList

9. 0.0 .

FirstList is Empty 0

0.

SecondList

9. 0 . 0 .

SecondList

9. 20. 0.

SecondList

20. 0 .

SecondList

20.

Whoops. The number of errors was 1

1 


\section{Appendix 4: Example Export Template for BlockList PIE}

\#

Define Variable: CellListlndex [Integer]

Define Variable: CellIndex [Integer]

Redirect output to: \$BaseName\$

Start a new line

Export expression: BL_InitializeGridInformation("Grid"); [G0]

Export expression: BL_AddVertexLayer("New Layer1"); [G0]

End line

Export expression: BL_AddVertexLayer("New Layer2"); [GO]

Start a new line

Export expression: "Number of Cell Lists: " [G0]

End line

Export expression: BL_GetCountOfCellLists() [G0]

Loop for: Variable CellListlndex from: 0 to: BL_GetCountOfCellLists()-1 step: 1

Start a new line

Export expression: "Cells for Object " [G0]

End line

Export expression: BL_CellListIndex [GO]

Loop for: Variable CellIndex from: 0 to: BL_GetCountOfACellList(CellListlndex)-1 step: 1 Start a new line

Export expression: "Column"; [GO]

Export expression: BL_GetCellColumn(CellListIndex, Celllndex); [GO]

Export expression: "Row"; [G0]

End line

Export expression: BL_GetCellRow(CellListlndex, CellIndex); [G0]

End loop

End loop

Start a new line

End line

Export expression: "Combined List" [GO]

Loop for: Variable CellIndex from: 0 to: BL_GetCountOfCombinedCellList()-1 step: 1

Start a new line

Export expression: "Column"; [GO]

Export expression: BL_GetCellColumnFromCombinedList(Celllndex); [G0]

Export expression: "Row"; [GO]

End line

Export expression: BL_GetCellRowFromCombinedList(Cellndex) [GO]

End loop

Start a new line

Export expression: "Relnitialize: "; [GO]

Export expression: BL_RelnitializeVertexList(); [GO]

End line

Export expression: BL_AddVertexLayer("New Layer3") [GO]

Start a new line

Export expression: "Number of Cell Lists: " [G0]

End line

Export expression: BL_GetCountOfCellLists() [GO]

Loop for: Variable CellListIndex from: 0 to: BL GetCountOfCellLists()-1 step: 1

Start a new line

Export expression: "Cells for Object " [G0]

End line

Export expression: CellListIndex [GO]

Loop for: Variable Celllndex from: 0 to: BL_GetCountOfACellList(CellListlndex)-1 step: 1 Start a new line

Export expression: "Column"; [GO]

Export expression: BL_GetCellColumn(CellListIndex, CellIndex); [GO]

Export expression: "Row"; [G0]

End line

Export expression: BL_GetCellRow(CellListlndex, CellIndex); [G0] 


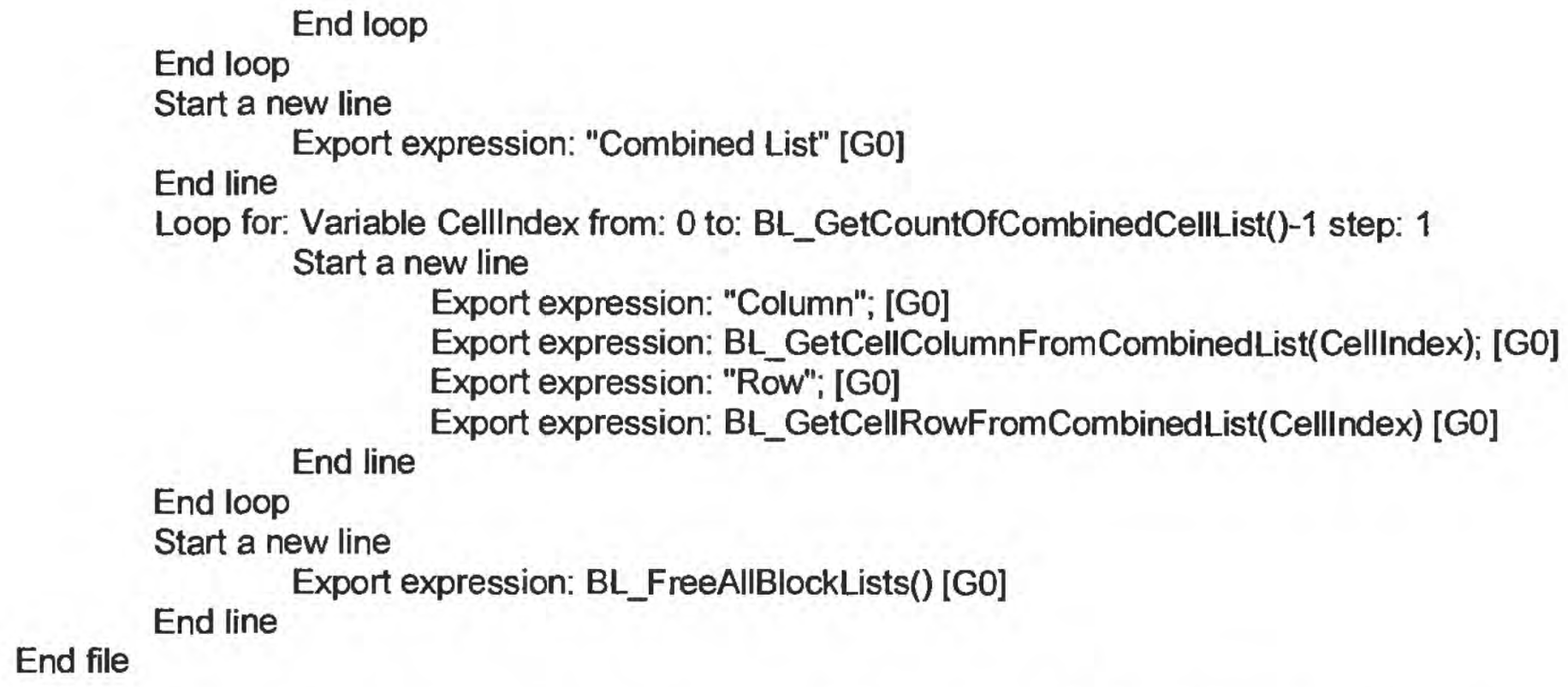

Executing this export template can yield a file with the following contents.

111

Number of Cell Lists: 2

Cells for Object 0

Column 1 Row 3

Column 2 Row 3

Column 3 Row 3

Column 4 Row 3

Column 5 Row 3

Column 6 Row 3

Column 6 Row 4

Cells for Object 1

Column 2 Row 5

Column 2 Row 4

Column 3 Row 4

Column 3 Row 3

Column 4 Row 3

Column 4 Row 2

Column 5 Row 2

Column 6 Row 2

Combined List

Column 1 Row 3

Column 2 Row 3

Column 3 Row 3

Column 4 Row 3

Column 5 Row 3

Column 6 Row 3

Column 6 Row 4

Column 2 Row 5

Column 2 Row 4

Column 3 Row 4

Column 4 Row 2

Column 5 Row 2

Column 6 Row 2

Relnitialize: 11

Number of Cell Lists: 1

Cells for Object 0

Column 1 Row 7

Column 2 Row 7

Column 3 Row 7

Column 4 Row 7

Column 5 Row 7 
Column 6 Row 7

Combined List

Column 1 Row 7

Column 2 Row 7

Column 3 Row 7

Column 4 Row 7

Column 5 Row 7

Column 6 Row 7

1 


\title{
Appendix 5: Export Templates Using Rotated $X$ and Rotated Y
}

\author{
Template 1 \\ \# \\ \# Export template to export the row and column numbers \\ \# and the locations of the four corners of each block. \\ \# \\ Define Variable: $X$ [Real] \\ Define Variable: $Y$ [Real] \\ \# \\ Redirect output to: \$BaseName\$ \\ Start a new line \\ Export expression: "Row Number"; [G0] \\ Export expression: "Column Number"; [G0] \\ Export expression: "X-coord 1"; [G0] \\ Export expression: "Y-coord 1"; [G0] \\ Export expression: "X-coord 2"; [G0] \\ Export expression: "Y-coord 2"; [G0] \\ Export expression: "X-coord 3"; [G0] \\ Export expression: "Y-coord 3"; [G0] \\ Export expression: "X-coord 4"; [G0] \\ End line \\ Export expression: "Y-coord 4"; [G0] \\ Loop for: Blocks \\ Start a new line \\ Export expression: Row(); [GO] \\ Export expression: Column(); [Go] \\ Set Variable: $X:=$ NthColumnPos(Column()-1) \\ Set Variable: $Y:=$ NthRowPos(Row()-1) \\ Export expression: Rotated X(X, Y, GridAngle()); [G0] \\ Export expression: Rotated $Y(X, Y$, GridAngle()); [GO] \\ Set Variable: $X:=$ NthColumnPos(Column()) \\ Export expression: Rotated X(X, Y, GridAngle()); [Go] \\ Export expression: Rotated $Y(X, Y$, GridAngle()); [G0] \\ Set Vanable: $Y:=$ NthRowPos(Row()) \\ Export expression: Rotated X(X, Y, GridAngle()); [G0] \\ Export expression: Rotated $Y(X, Y$, GridAngle()); [G0] \\ Set Variable: $X:=$ NthColumnPos(Column()-1) \\ Export expression: Rotated X(X, Y, GridAngle()); [G0] \\ Export expression: Rotated $Y(X, Y$, GridAngle()); [Go] \\ End line
}

End file

End loop 


\section{Template 2}

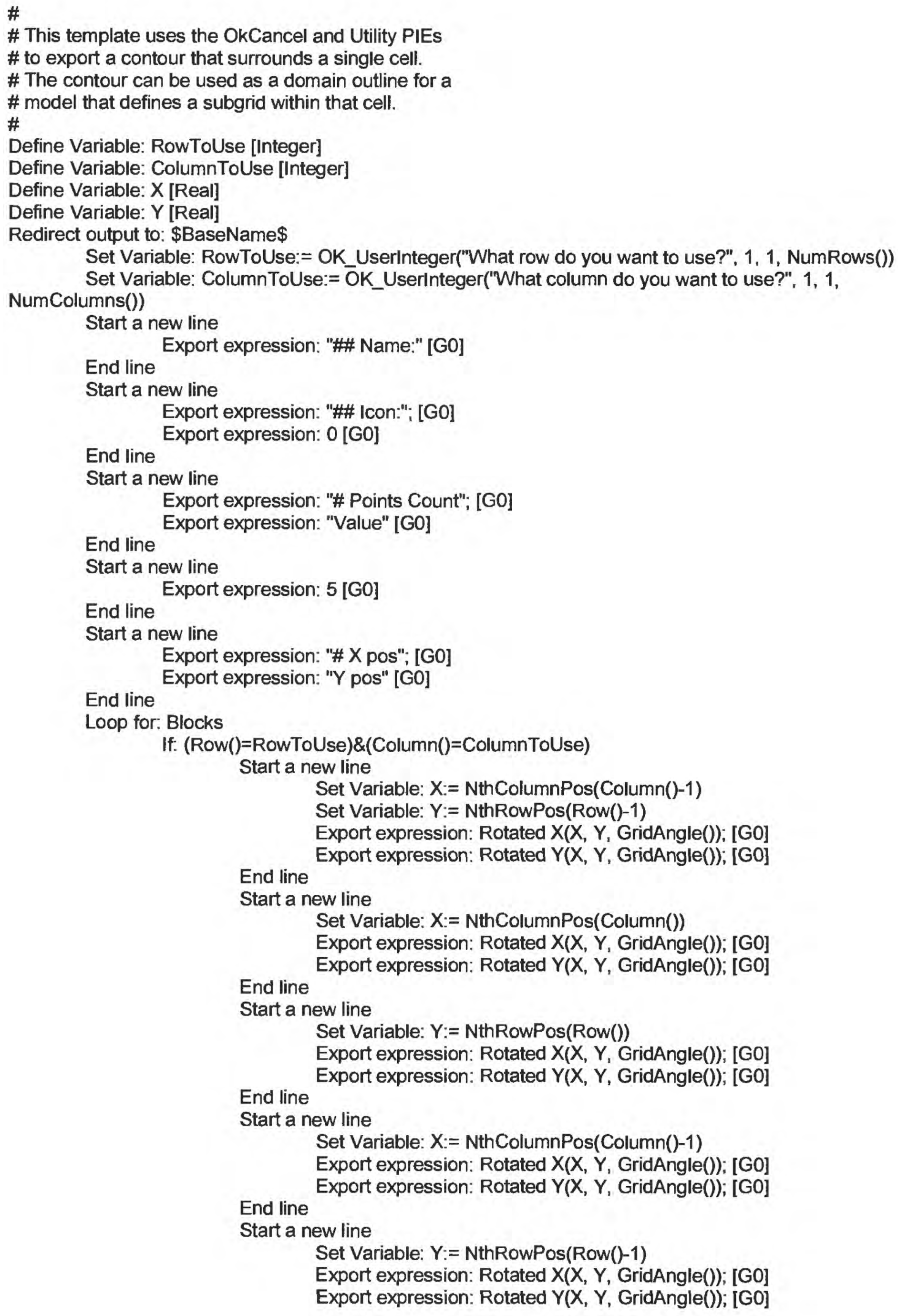




End loop End if End line

End file 


\section{Appendix 6: DEM2Image.exe}

DEM2Image.exe reads Digital Elevation Models (DEMs) in the format described in the DEM data users guide (U.S. Department of the Interior, U.S. Geological Survey, 1992) (http://rockyweb.cr.usgs.gov/nmpstds/demstds.html) and displays them as bitmaps. Once the program has been started by double-clicking on its icon, its operation is much the same as the Sample DEM Data command described in the main body of this report. However, you do not need to specify a grid nor does DEM2Image extract gridded data. 